\title{
The Total Synthesis of Moenomycin A
}

\author{
James G. Taylor ${ }^{\dagger}$, Xuechen $\mathrm{Li}^{\dagger}$, Markus Oberthür ${ }^{\dagger}$, Wenjiang Zhu ${ }^{\dagger}$, and Daniel E. Kahne ${ }^{\dagger \dagger *}$ \\ Department of Chemistry and Chemical Biology, Harvard University \\ 12 Oxford Street, Cambridge, MA 02138 \\ Departments of Biological Chemistry and Molecular Biology, \\ Harvard Medical School, Boston, MA 02115
}

\section{Supporting Information}

S1- Experimental Procedures

S40- References

S41- NMR Spectra 


\section{General Methods.}

Unless otherwise noted, all reactions were conducted under an argon atmosphere using anhydrous solvents (either distilled or passed through an activated alumina column). Commercially available reagents were used without further purification. Thin layer chromatography (TLC) was performed using glass plates coated with silica gel $(250 \mu \mathrm{m}$, Sorbent Technologies), with detection by UV and $p$ anisaldehyde. Flash chromatography was carried out on silica gel (60 $\AA, 32-63 \mu \mathrm{m})$ purchased from Sorbent Technologies. Analytical HPLC was performed on a Hewlett-Packard 1100 series instrument using a Phenomenex Luna $5 \mu \mathrm{m} \mathrm{C18} \mathrm{column}(250 \mathrm{~mm}$ x $4.6 \mathrm{~mm})$. Preparative HPLC was performed on a Hitachi L6200 instrument using a Phenomenex Luna 5 um C18 column (250 x $21.2 \mathrm{~mm})$.

NMR spectra were recorded on a Varian Inova $400\left(400 \mathrm{MHz}\right.$ for ${ }^{1} \mathrm{H}, 100 \mathrm{MHz}$ for $\left.{ }^{13} \mathrm{C}\right)$, Varian Inova $500\left(500 \mathrm{MHz}\right.$ for ${ }^{1} \mathrm{H}, 125 \mathrm{MHz}$ for $\left.{ }^{13} \mathrm{C}\right)$, Varian Inova $600\left(600 \mathrm{MHz}\right.$ for $\left.{ }^{1} \mathrm{H}\right)$, or Bruker DMX 500 (500 MHz for ${ }^{1} \mathrm{H}, 125 \mathrm{MHz}$ for ${ }^{13} \mathrm{C}$ ) spectrometer. Proton chemical shifts are reported in parts per million (ppm) on the $\delta$ scale from an internal standard of residual protium in the NMR solvents $\left(\mathrm{CHCl}_{3}: \delta\right.$ 7.26, $\mathrm{D}_{2} \mathrm{HCOD} 3.30$ ). Data for ${ }^{13} \mathrm{C} \mathrm{NMR}$ are reported in terms of chemical shift from the carbon resonances of the solvent $\left(\mathrm{CDCl}_{3}: \delta 77.16, \mathrm{CD}_{3} \mathrm{OD} 49.0\right)$. Data are reported as follows: chemical shift, multiplicity ( $\mathrm{s}=$ singlet, $\mathrm{d}=$ doublet, $\mathrm{t}=$ triplet, $\mathrm{q}=$ quartet, $\mathrm{m}=$ multiplet, $\mathrm{br}=$ broad $)$, coupling constant in $\mathrm{Hz}$, integration, and assignment. A peak designated as "ABq" in the ${ }^{1} \mathrm{H}$ NMR spectrum indicates it was one partner in an $\mathrm{AB}$ quartet. Low resolution mass spectra (LRMS) were obtained on an Agilent Technologies LC/MSD instrument (Model \#G1956B) using electrospray ionization (ESI), while high resolution mass spectra (HRMS), ESI mode, were obtained at the Harvard University Mass Spectrometry Facilities. 
Synthesis of compound 2 .

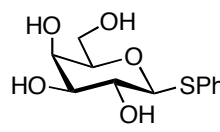

2.1
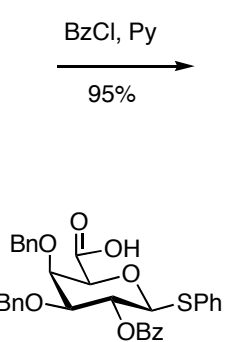

2.6
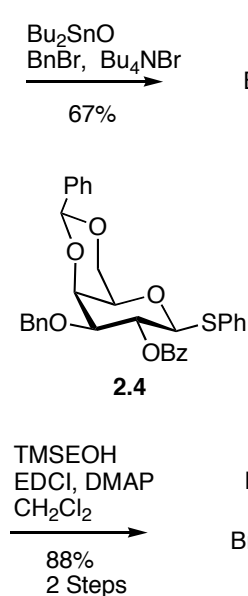

2 Steps

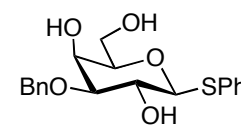

2.2

\section{$\mathrm{BH}_{3} \cdot$ THF} $\mathrm{Bu}_{2} \mathrm{BOTf}$

$\underset{90 \%}{\stackrel{\mathrm{THF}}{\longrightarrow}}$

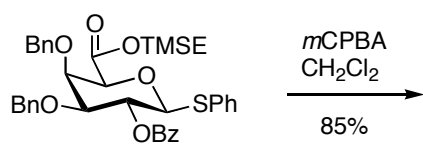

2.7

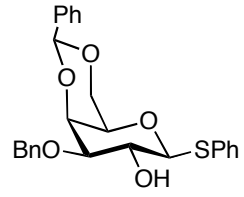

2.3

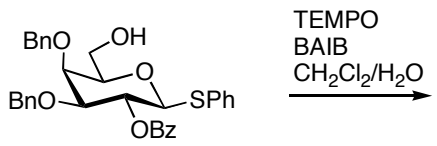

2.5

Synthesis of compound $\mathbf{3}$.

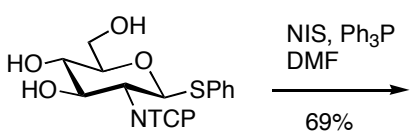

3.1

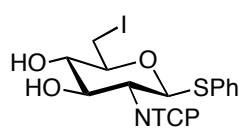

3.2

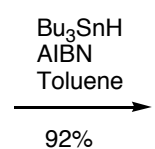

$92 \%$

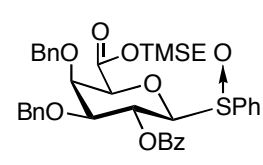

Synthesis of compound 4 .

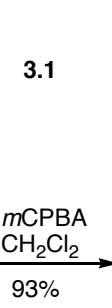

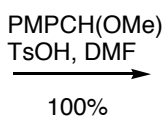

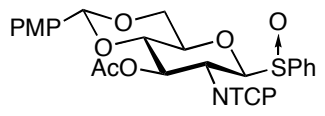

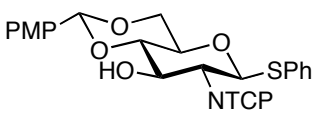

4.1

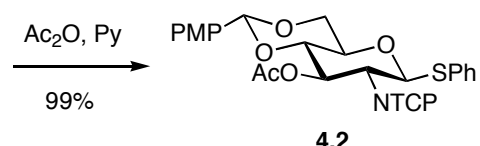

4.2 
Synthesis of compound $\mathbf{5}$.
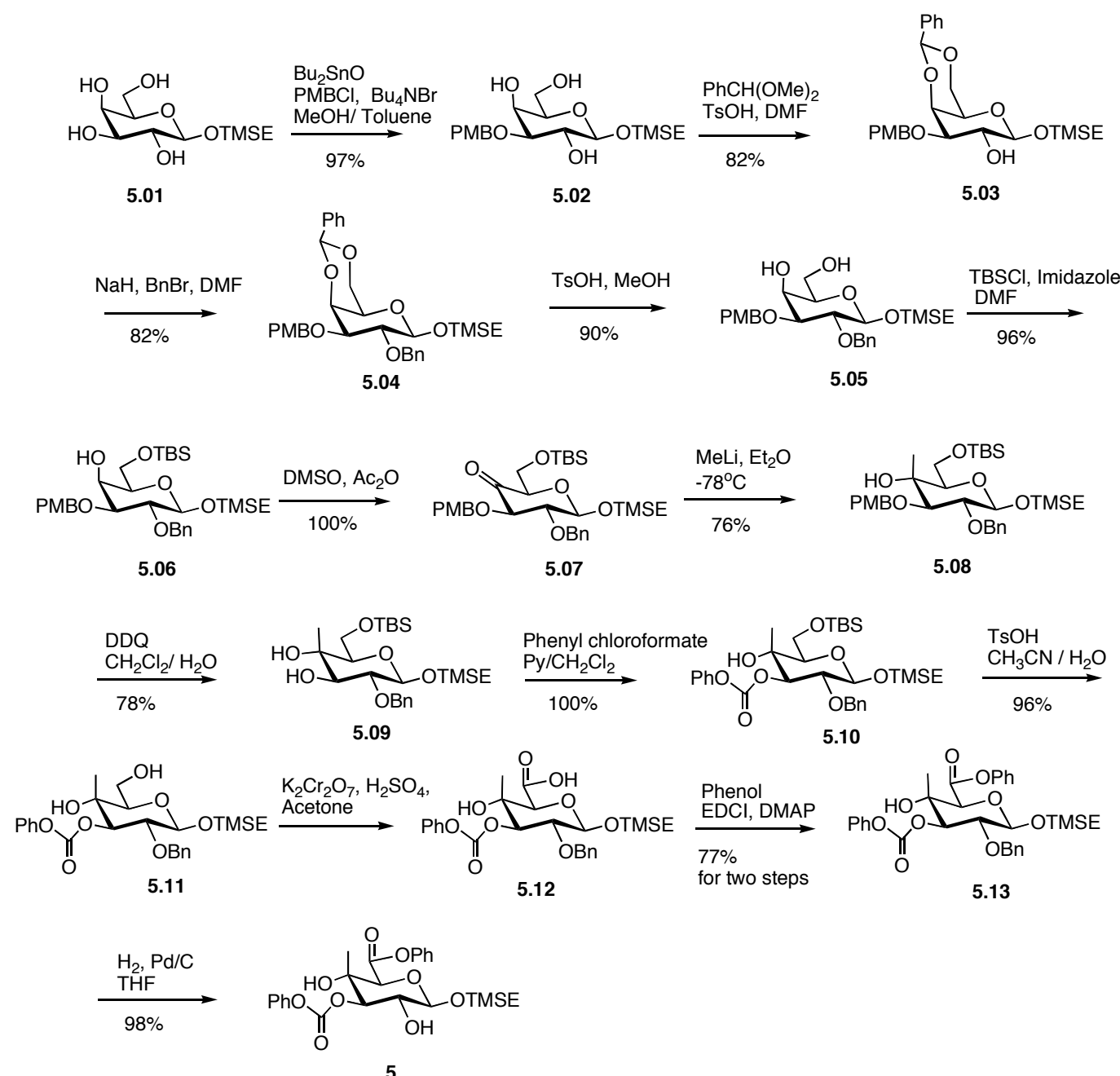


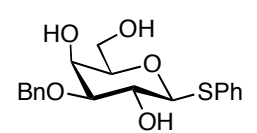

Phenyl 3- $O$-benzyl-1-thio- $\beta$-D-galactopyranoside (2.2).

A solution of phenyl 1-thio- $\beta$-D-galactopyranoside (2.1) $(3.00 \mathrm{~g}, 11.0 \mathrm{mmol})$ and dibutyltin oxide $(3.00 \mathrm{~g}$, $12.1 \mathrm{mmol})$ in dry methanol $(80 \mathrm{ml})$ was refluxed for $2 \mathrm{~h}$ to produce a clear mixture. The solvent was then evaporated under reduced pressure. The resultant residue was dissolved in dry benzene $(80 \mathrm{~mL})$ followed by the addition of $\mathrm{Bu}_{4} \mathrm{NBr}(3.90 \mathrm{~g}, 12.11 \mathrm{mmol})$ and $\mathrm{BnBr}(1.3 \mathrm{ml}, 12.1 \mathrm{mmol})$. The mixture was refluxed overnight. After cooling to room temperature, the mixture was concentrated and the residue was purified by silica gel chromatography $(50 \%$ EtOAc/petroleum ether) to afford the title compound $(2.71 \mathrm{~g}, 7.48$ mmol, 67\%). ${ }^{1} \mathrm{H}$ NMR (400 MHz, $\left.\mathrm{CDCl}_{3}\right): \delta 7.60-7.20(\mathrm{~m}, 10 \mathrm{H}, \mathrm{PhH}), 4.77,4.68(\mathrm{ABq}, J=11.7 \mathrm{~Hz}, 1$ $\mathrm{H}$ each, $\left.\mathrm{OCH}_{2} \mathrm{Ph}\right), 4.59(\mathrm{~d}, J=9.5 \mathrm{~Hz}, 1 \mathrm{H}, \mathrm{H}-1), 4.08(\mathrm{~d}, J=2.6 \mathrm{~Hz}, 1 \mathrm{H}, \mathrm{H}-4), 3.76(\mathrm{dd}, J=9.5,9.5 \mathrm{~Hz}$, $1 \mathrm{H}, \mathrm{H}-2), 3.75$ (dd, $J=11.4,5.8 \mathrm{~Hz}, 1 \mathrm{H}, \mathrm{H}-6), 3.70$ (dd, $J=11.4,5.8 \mathrm{~Hz}, 1 \mathrm{H}, \mathrm{H}-6), 3.50$ (dd, $J=5.8$, $5.8 \mathrm{~Hz}, 1 \mathrm{H}, \mathrm{H}-5), 3.41$ (dd, $J=9.5,2.6 \mathrm{~Hz}, 1 \mathrm{H}, \mathrm{H}-3) ;{ }^{13} \mathrm{C} \mathrm{NMR}\left(100 \mathrm{MHz}, \mathrm{CDCl}_{3}\right): \delta$ 131.0, 128.6, $128.1,127.8,127.4,126.8,89.1,82.5,79.2,71.5,69.0,66.2,61.4$; HRMS calcd for $\mathrm{C}_{19} \mathrm{H}_{26} \mathrm{O}_{5} \mathrm{SN}[\mathrm{M}+$ $\left.\mathrm{NH}_{4}\right]^{+}: 380.1532$, found 380.1527 .

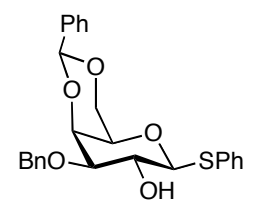

\section{Phenyl 3-O-benzyl-4,6-O-benzylidene-1-thio- $\beta$-D-galactopyranoside (2.3).}

To a solution of $2.2(2.71 \mathrm{~g}, 7.48 \mathrm{mmol})$ in dry $\mathrm{CH}_{3} \mathrm{CN}$ was successively added benzaldehyde dimethylacetal $(1.35 \mathrm{ml}, 9.72 \mathrm{mmol})$ and $\mathrm{TsOH} \cdot \mathrm{H}_{2} \mathrm{O}(0.42 \mathrm{~g}, 1.49 \mathrm{mmol})$. After $3 \mathrm{~h}$, the reaction was cooled to $0{ }^{\circ} \mathrm{C}$ and triethylamine $(2 \mathrm{ml})$ was added to neutralize the reaction mixture at $0{ }^{\circ} \mathrm{C}$. After evaporation of the solvent, the curde residue was purified by silica gel chromatography $(20 \%$ EtOAc/toluene) to afford the title compound $(3.10 \mathrm{~g}, 6.88 \mathrm{mmol}, 92 \%) .{ }^{1} \mathrm{H}$ NMR $\left(500 \mathrm{MHz}, \mathrm{CDCl}_{3}\right): \delta$ $7.70-7.20$ (m, $15 \mathrm{H}, \mathrm{PhH}), 5.42$ (s, $1 \mathrm{H}, \mathrm{CHPh}), 4.73,4.69$ (ABq, $J=12.2 \mathrm{~Hz}, 1 \mathrm{H}$ each, $\left.\mathrm{OCH}_{2} \mathrm{Ph}\right), 4.51$ 
(d, $J=9.8 \mathrm{~Hz}, 1 \mathrm{H}, \mathrm{H}-1), 4.33$ (d, $J=12.2 \mathrm{~Hz}, 1 \mathrm{H}, \mathrm{H}-6), 4.13$ (d, $J=3.0 \mathrm{~Hz}, 1 \mathrm{H}, \mathrm{H}-4), 3.96$ (d, $J=12.2$ $\mathrm{Hz}, 1 \mathrm{H}, \mathrm{H}-6), 3.84$ (dd, $J=9.8,9.8 \mathrm{~Hz}, 1 \mathrm{H}, \mathrm{H}-2), 3.49$ (dd, $J=9.8,3.0 \mathrm{~Hz}, 1 \mathrm{H}, \mathrm{H}-3), 3.41$ (s, $1 \mathrm{H}, \mathrm{H}-$ 5); ${ }^{13} \mathrm{C}$ NMR (125 MHz, $\left.\mathrm{CDCl}_{3}\right): \delta 133.9,129.3,129.1,128.7,128.4,128.3,128.1,126.7,101.4,87.3$, 80.5, 73.5, 71.8, 70.3, 69.6, 67.4; HRMS calcd for $\mathrm{C}_{26} \mathrm{H}_{30} \mathrm{O}_{5} \mathrm{SN}\left[\mathrm{M}+\mathrm{NH}_{4}\right]^{+}: 468.1835$, found 468.1845.

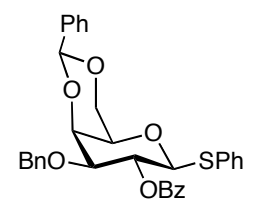

Phenyl 2- $O$-benzoyl-3- $O$-benzyl-4,6- $O$-benzylidene-1-thio- $\beta$-D-galactopyranoside (2.4).

To a solution of $\mathbf{2 . 3}(1.85 \mathrm{~g}, 4.11 \mathrm{mmol})$ in dry pyridine $(10 \mathrm{ml})$ was added benzoyl chloride $(0.95 \mathrm{ml}$, $4.22 \mathrm{mmol}$ ). The mixture was stirred at room temperature overnight then diluted with EtOAc and washed sequentially with water and brine, then the organic phase dried over $\mathrm{Na}_{2} \mathrm{SO}_{4}$. After evaporation of the solvent, the crude residue was purified by silica gel chromatography (20\% EtOAc/toluene) to afford the title compound (2.16 g, $3.89 \mathrm{mmol}, 95 \%) .{ }^{1} \mathrm{H}$ NMR (400 MHz, $\left.\mathrm{CDCl}_{3}\right): \delta 8.20-7.20(\mathrm{~m}, 20 \mathrm{H}, \mathrm{PhH})$, $5.70(\mathrm{dd}, J=9.9,9.9 \mathrm{~Hz}, 1 \mathrm{H}, \mathrm{H}-2), 5.52(\mathrm{~s}, 1 \mathrm{H}, \mathrm{CHPh}), 4.87$ (d, $J=9.9 \mathrm{~Hz}, 1 \mathrm{H}, \mathrm{H}-1), 4.71,4.60$ (ABq, $J=12.8 \mathrm{~Hz}, 1 \mathrm{H}$ each, $\left.\mathrm{OCH}_{2} \mathrm{Ph}\right), 4.36(\mathrm{~d}, J=11.5 \mathrm{~Hz}, 1 \mathrm{H}, \mathrm{H}-6), 4.32$ (d, $\left.J=2.9 \mathrm{~Hz}, 1 \mathrm{H}, \mathrm{H}-4\right), 4.04$ (d, $J=11.5 \mathrm{~Hz}, 1 \mathrm{H}, \mathrm{H}-6), 3.84(\mathrm{dd}, J=9.9,2.9 \mathrm{~Hz}, 1 \mathrm{H}, \mathrm{H}-3), 3.48$ (s, $1 \mathrm{H}, \mathrm{H}-5) ;{ }^{13} \mathrm{C}$ NMR (100 MHz, $\left.\mathrm{CDCl}_{3}\right): \delta 165.4,138.2,138.0,133.6,132.4,130.1,129.4,129.1,128.7,128.4,128.0,126.9,101.4,85.9$, 78.5, 73.2, 71.1, 70.3, 69.5, 69.4; HRMS calcd for $\mathrm{C}_{33} \mathrm{H}_{34} \mathrm{O}_{6} \mathrm{SN}\left[\mathrm{M}+\mathrm{NH}_{4}\right]^{+}:$572.2107, found, 572.2089.

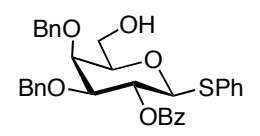

\section{Phenyl 2- $O$-benzoyl-3,4-di- $O$-benzyl-1-thio- $\beta$-D-galactopyranoside (2.5).}

A solution of $1 \mathrm{M} \mathrm{BH}_{3} \bullet$ THF (30 ml, $\left.30.00 \mathrm{mmol}\right)$ was added to compound 2.4 (1.61 g, $\left.2.90 \mathrm{mmol}\right)$, at $0{ }^{\circ} \mathrm{C}$, and then a solution of $1 \mathrm{M} \mathrm{Bu}_{2} \mathrm{BOTf}$ in $\mathrm{CH}_{2} \mathrm{Cl}_{2}(3.0 \mathrm{ml}, 3.00 \mathrm{mmol})$ was added to the reaction slowly. After 1 hour at $0{ }^{\circ} \mathrm{C}$, triehylamine was added followed by careful addition of methanol until the 
evolution of $\mathrm{H}_{2}$ ceased. The crude mixture was then concentrated and purified by silica gel chromatography (25\% EtOAc/petroleum ether) to afford the title compound (1.45 g, $2.60 \mathrm{mmol}, 90 \%) .{ }^{1} \mathrm{H}$ $\operatorname{NMR}\left(400 \mathrm{MHz}, \mathrm{CDCl}_{3}\right): \delta 8.10-7.20(\mathrm{~m}, 20 \mathrm{H}, \mathrm{PhH}), 5.71(\mathrm{dd}, J=9.9,9.9 \mathrm{~Hz}, 1 \mathrm{H}, \mathrm{H}-2), 5.00,4.64$ $\left(\mathrm{ABq}, J=12.0 \mathrm{~Hz}, 1 \mathrm{H}\right.$ each, $\left.\mathrm{OCH}_{2} \mathrm{Ph}\right), 4.79(\mathrm{~d}, J=9.9 \mathrm{~Hz}, 1 \mathrm{H}, \mathrm{H}-1), 4.68,4.55(\mathrm{ABq}, J=12.0 \mathrm{~Hz}, 1 \mathrm{H}$ each, $\left.\mathrm{OCH}_{2} \mathrm{Ph}\right), 4.93$ (d, $\left.J=2.5 \mathrm{~Hz}, 1 \mathrm{H}, \mathrm{H}-4\right), 3.85$ (m, $\left.1 \mathrm{H}, \mathrm{H}-6\right), 3.72$ (dd, $\left.J=9.9,2.5 \mathrm{~Hz}, 1 \mathrm{H}, \mathrm{H}-3\right)$, 3.60 - 3.53 (m, $2 \mathrm{H}, \mathrm{H}-5,6) ;{ }^{13} \mathrm{C}$ NMR $\left(100 \mathrm{MHz}, \mathrm{CDCl}_{3}\right): \delta 165.5,138.3,133.6,133.3,132.2,129.0$, 128.6, 128.0, 127.8, 87.1, 81.5, 79.2, 74.3, 72.4, 70.6, 62.4; LRMS calcd for $\mathrm{C}_{33} \mathrm{H}_{33} \mathrm{O}_{6} \mathrm{~S}[\mathrm{M}+\mathrm{H}]^{+}: 557.2$, found, 557.2.

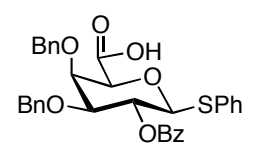

\section{Phenyl 3,4-di- $O$-benzyl-2- $O$-benzoyl-1-thio- $\beta$-D-galactopyranosiduronic acid (2.6).}

To a vigorously stirred solution of compound 2.5 (1.40 g, $2.51 \mathrm{mmol})$ in $10 \mathrm{ml} \mathrm{CH}_{2} \mathrm{Cl}_{2}$ and $5 \mathrm{ml} \mathrm{H}_{2} \mathrm{O}$ was added TEMPO (78 $\mathrm{mg}, 0.50 \mathrm{mmol})$ and BAIB $(2.00 \mathrm{~g}, 6.21 \mathrm{mmol})$. The reaction mixture was stirred for $1 \mathrm{~h}$ then quenched by the addition of $10 \% \mathrm{Na}_{2} \mathrm{~S}_{2} \mathrm{O}_{3}$ solution and extracted twice with EtOAc. The combined organic layers were washed with brine and then dried over $\mathrm{Na}_{2} \mathrm{SO}_{4}$. After evaporation of the solvent, the crude residue was subjected to the next step without further purification. $\left(500 \mathrm{MHz}, \mathrm{CDCl}_{3}\right)$ :

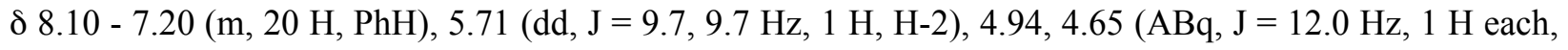
$\left.\mathrm{OCH}_{2} \mathrm{Ph}\right), 4.83(\mathrm{~d}, \mathrm{~J}=9.7 \mathrm{~Hz}, 1 \mathrm{H}, \mathrm{H}-1), 4.65,4.50\left(\mathrm{ABq}, \mathrm{J}=12.0 \mathrm{~Hz}, 1 \mathrm{H}\right.$ each, $\left.\mathrm{OCH}_{2} \mathrm{Ph}\right), 4.44(\mathrm{~s}, 1 \mathrm{H}$, H-4), 4.18 (s, $1 \mathrm{H}, \mathrm{H}-5), 3.78$ (br d, J = 9.7 Hz, $1 \mathrm{H}, \mathrm{H}-3$ ). HRMS calcd for $\mathrm{C}_{33} \mathrm{H}_{34} \mathrm{O}_{7} \mathrm{SN}\left[\mathrm{M}+\mathrm{NH}_{4}\right]^{+}$: 588.2056, found 588.2034.

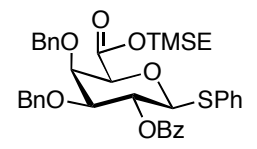


2-(Trimethylsilyl)ethyl (phenyl 3,4-di- $O$-benzyl-2- $O$-benzoyl-1-thio- $\beta$-D-galactopyrosid) uronate (2.7).

A solution of the above residue, EDCI (0.58 g, $3.02 \mathrm{mmol})$, DMAP (46 $\mathrm{mg}, 0.38 \mathrm{mmol})$ and 2(trimethylsilyl)ethanol $(0.89 \mathrm{ml}, 6.25 \mathrm{mmol})$ in $\mathrm{CH}_{2} \mathrm{Cl}_{2}(10 \mathrm{ml})$ was stirred at room temperature for $2 \mathrm{~h}$. The reaction mixture was then diluted with $\mathrm{CH}_{2} \mathrm{Cl}_{2}$, washed with water and brine, then the organic phase was dried over $\mathrm{Na}_{2} \mathrm{SO}_{4}$. After evaporation of the solvent, the residue was purified by silica gel chromatography (20\% EtOAc/ petroleum ether) to afford the title compound (1.48 g, $2.21 \mathrm{mmol}, 88 \%$ over 2 steps). ${ }^{1} \mathrm{H}$ NMR (400 MHz, $\left.\mathrm{CDCl}_{3}\right): \delta 8.10-7.10(\mathrm{~m}, 20 \mathrm{H}, \mathrm{PhH}), 5.71(\mathrm{dd}, J=10.0,10.0 \mathrm{~Hz}, 1$ H, H-2), 4.98, $4.64\left(\mathrm{ABq}, J=11.7 \mathrm{~Hz}, 1 \mathrm{H}\right.$ each, $\left.\mathrm{OCH}_{2} \mathrm{Ph}\right), 4.77$ (d, $\left.J=10.0 \mathrm{~Hz}, 1 \mathrm{H}, \mathrm{H}-1\right), 4.67,4.53$ (ABq, $J=12.2 \mathrm{~Hz}, 1 \mathrm{H}$ each, $\left.\mathrm{OCH}_{2} \mathrm{Ph}\right), 4.42(\mathrm{dd}, J=2.6,1.1 \mathrm{~Hz}, 1 \mathrm{H}, \mathrm{H}-4), 4.30-4.16(\mathrm{~m}, 2 \mathrm{H}$, $\mathrm{OCH}_{2} \mathrm{CH}_{2} \mathrm{SiMe}_{3}$ ), 4.13 (d, $\left.J=1.1 \mathrm{~Hz}, 1 \mathrm{H}, \mathrm{H}-5\right), 3.78$ (dd, $\left.J=10.0,2.6 \mathrm{~Hz}, 1 \mathrm{H}, \mathrm{H}-3\right), 1.00$ (m, $2 \mathrm{H}$, $\mathrm{OCH}_{2} \mathrm{CH}_{2} \mathrm{SiMe}_{3}$ ), 0.02 (s, $\left.9 \mathrm{H}, \mathrm{SiMe}_{3}\right) ;{ }^{13} \mathrm{C} \mathrm{NMR}\left(100 \mathrm{MHz}, \mathrm{CDCl}_{3}\right): \delta 168.1,165.4,138.4,137.5,133.4$, $133.3,130.3,128.9,128.6,128.1,128.0,127.7,87.0,80.6,77.8,74.7,72.1,70.0,64,4,60.4,22.4,17.6$, 1.1, -1.3; HRMS calcd for $\mathrm{C}_{38} \mathrm{H}_{46} \mathrm{O}_{7} \mathrm{SSiN}\left[\mathrm{M}+\mathrm{NH}_{4}\right]^{+}:$688.2764, found 688.2798.

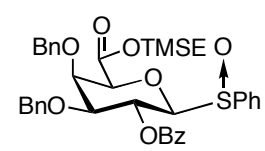

2-(Trimethylsilyl)ethyl (phenyl 3,4-di- $O$-benzyl-2- $O$-benzoyl-1-thio- $\beta$-D-galactopyrosid) uronate $S$ Oxide (2).

To a solution of $2.7(850 \mathrm{mg}, 1.24 \mathrm{mmol})$ in $\mathrm{CH}_{2} \mathrm{Cl}_{2}(5 \mathrm{ml})$ was added $\mathrm{mCPBA}(307 \mathrm{mg}$ of $75 \%$ dispersion, $1.33 \mathrm{mmol}$ ) at $-78{ }^{\circ} \mathrm{C}$. The suspension was gradually warmed to $0{ }^{\circ} \mathrm{C}$ over $2 \mathrm{~h}$ then quenched with saturated $\mathrm{NaHCO}_{3}$ and extracted with $\mathrm{CH}_{2} \mathrm{Cl}_{2}$. The organic layer was washed with brine then dried over $\mathrm{Na}_{2} \mathrm{SO}_{4}$. After evaporation of the solvent, the crude residue was purified by silica gel chromatography (30\% EtOAc/petroleum ether) to afford the title compound (723 mg, $1.05 \mathrm{mmol}, 85 \%)$. ${ }^{1} \mathrm{H}$ NMR $\left(400 \mathrm{MHz}, \mathrm{CDCl}_{3}\right): \delta 8.00-7.00(\mathrm{~m}, 20 \mathrm{H}, \mathrm{PhH}), 5.74(\mathrm{dd}, J=9.6,9.6 \mathrm{~Hz}, 1 \mathrm{H}, \mathrm{H}-2), 4.85$, 
$4.46\left(\mathrm{ABq}, J=11.7 \mathrm{~Hz}, 1 \mathrm{H}\right.$ each, $\left.\mathrm{OCH}_{2} \mathrm{Ph}\right), 4.66,4.52\left(\mathrm{ABq}, J=12.2 \mathrm{~Hz}, 1 \mathrm{H}\right.$ each, $\left.\mathrm{OCH}_{2} \mathrm{Ph}\right), 4.56(\mathrm{~d}$, $J=9.6 \mathrm{~Hz}, 1 \mathrm{H}, \mathrm{H}-1), 4.32(\mathrm{dd}, J=2.6,1.5 \mathrm{~Hz}, 1 \mathrm{H}, \mathrm{H}-4), 4.13(\mathrm{~d}, J=1.5 \mathrm{~Hz}, 1 \mathrm{H}, \mathrm{H}-5), 4.14$ - 4.02 (m, $\left.2 \mathrm{H}, \mathrm{OCH}_{2} \mathrm{CH}_{2} \mathrm{SiMe}_{3}\right), 3.82(\mathrm{dd}, \mathrm{J}=9.6,2.6 \mathrm{~Hz}, 1 \mathrm{H}, \mathrm{H}-3), 0.96\left(\mathrm{~m}, 2 \mathrm{H}, \mathrm{OCH}_{2} \mathrm{CH}_{2} \mathrm{SiMe}_{3}\right), 0.02(\mathrm{~s}, 9 \mathrm{H}$, $\left.\mathrm{SiMe}_{3}\right) ;{ }^{13} \mathrm{C} \mathrm{NMR}\left(100 \mathrm{MHz}, \mathrm{CDCl}_{3}\right): \delta$ 167.6, 165.5, 138.8, 138.2, 137.2, 133.6, 131.7, 130.2, 129.6, $128.7,128.3,127.9,127.5,127.2,92.8,79.8,77.8,74.6,74.5,72.1,67.7,64.3,17.7,-1.3$. HRMS calcd for $\mathrm{C}_{38} \mathrm{H}_{43} \mathrm{O}_{8} \mathrm{SSi}[\mathrm{M}+\mathrm{H}]^{+}:$687.2448, found 687.2457.

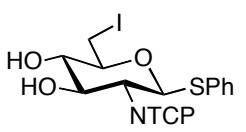

Phenyl 6-Iodo-2-deoxy-2-tetrachlorophthalimido-1-thio- $\beta$-D-glucopyranoside (3.2).

To a stirred solution of phenyl 2-deoxy-2-tetrachlorophthalimido-1-thio- $\beta$-D-glucopyranoside ${ }^{1}$ (3.1) (440mg, $0.816 \mathrm{mmol})$ in DMF $(7 \mathrm{~mL})$ at $0{ }^{\circ} \mathrm{C}$ was added $N$-iodosuccinimide (404 $\mathrm{mg}, 1.80 \mathrm{mmol}$ ). Triphenylphosphine $(471 \mathrm{mg}, 1.80 \mathrm{mmol})$ in DMF $(5 \mathrm{~mL})$ was then added dropwise over 30 min followed by heating of the reaction mixture to $50{ }^{\circ} \mathrm{C}$ for an additional $30 \mathrm{~min}^{2}$ After concentration in vacuo, the crude mixture was purified by silica gel chromatography $(30 \% \mathrm{EtOAc} /$ petroleum ether) to afford the title compound (367 mg, $0.565 \mathrm{mmol}, 69 \%) .{ }^{1} \mathrm{H} \mathrm{NMR}\left(500 \mathrm{MHz}, 10 \% \mathrm{CD}_{3} \mathrm{OD} / \mathrm{CDCl}_{3}\right) \delta \mathrm{ppm} 7.45(\mathrm{dd}, J=$ 6.6, 3.2 Hz, 2 H, PhH), 7.23 - 7.19 (m, 3 H, PhH), 5.50 (d, J=10.3 Hz, 1 H, H-1), 4.19 - 4.13 (m, 1 H, H3), 4.08 (dd, $J=10.3,10.3 \mathrm{~Hz}, 1 \mathrm{H}, \mathrm{H}-2), 3.61$ (d, $J=10.3 \mathrm{~Hz}, 1 \mathrm{H}, \mathrm{H}-6), 3.36$ - 3.30 (m, $1 \mathrm{H}, \mathrm{H}-4), 3.26$

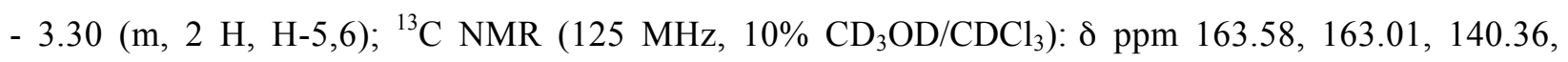
$132.85,131.48,130.04,129.68,128.92,128.11,127.28,127.22,82.98,79.01,74.82,71.78,56.30,5.81$; HRMS calcd for $\mathrm{C}_{20} \mathrm{H}_{18} \mathrm{Cl}_{4} \mathrm{IN}_{2} \mathrm{O}_{5} \mathrm{~S}\left[\mathrm{M}+\mathrm{NH}_{4}\right]^{+}: 664.8735$, found 664.8713 .

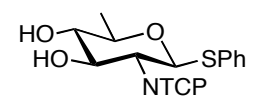

Phenyl 2,6-Dideoxy-2-tetrachlorophthalimido-1-thio- $\beta$-D-glucopyranoside (3). 
Compound 3.2 (367 mg, $0.565 \mathrm{mmol})$ was dried by azeotropic distillation with toluene $(3 \mathrm{x} 10 \mathrm{~mL})$ then suspended in toluene and heated to $70^{\circ} \mathrm{C} .2,2^{\prime}$-Azobis(2-methylpropionitrile) (70 $\left.\mathrm{mg}, 0.426 \mathrm{mmol}\right)$ was added followed by tributyltin hydride $(941 \mu \mathrm{L}, 3.40 \mathrm{mmol})$ and the reaction stirred for $1 \mathrm{~h}^{2}$ After cooling to room temperature, the mixture was concentrated in vacuo then separated by silica gel chromatography (40\% EtOAc/petroleum ether) to afford the title compound (271 mg, $0.518 \mathrm{mmol}, 92 \%$ yield). ${ }^{1} \mathrm{H}$ NMR $\left(600 \mathrm{MHz}, \mathrm{CDCl}_{3}\right): \delta \mathrm{ppm} 7.35(\mathrm{dd}, J=7.8,1.6 \mathrm{~Hz}, 2 \mathrm{H}, \mathrm{PhH}), 7.26-7.21(\mathrm{~m}, 3 \mathrm{H}, \mathrm{PhH}), 5.49(\mathrm{~d}, J=$ $10.3 \mathrm{~Hz}, 1 \mathrm{H}, \mathrm{H}-1), 4.19$ (dd, $J=9.5,9.5 \mathrm{~Hz}, 1 \mathrm{H}, \mathrm{H}-3$ ), 4.10 (dd, $J=10.4,10.4 \mathrm{~Hz}, 1 \mathrm{H}, \mathrm{H}-2), 3.56$ 3.50 (m, $1 \mathrm{H}, \mathrm{H}-5), 3.16(\mathrm{dd}, J=8.9,8.9 \mathrm{~Hz}, 1 \mathrm{H}, \mathrm{H}-4), 1.35$ (d, $J=6.2 \mathrm{~Hz}, 3 \mathrm{H}, \mathrm{H}-6) ;{ }^{13} \mathrm{C}$ NMR $(125$ $\left.\mathrm{MHz}, \mathrm{CDCl}_{3}\right): \delta$ ppm 163.76, 163.25, 140.52, 140.34, 132.25, 131.79, 130.50, 129.56, 129.00, 127.78, 127.38, 127.13, 82.62, 76.89, 76.16, 71.79, 56.66, 17.93; HRMS calcd for $\mathrm{C}_{20} \mathrm{H}_{19} \mathrm{Cl}_{4} \mathrm{~N}_{2} \mathrm{O}_{5} \mathrm{~S}\left[\mathrm{M}+\mathrm{NH}_{4}\right]^{+}$: 538.9769, found 538.9786.

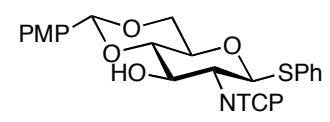

\section{Phenyl 2-Deoxy-4,6- $O$-p-methoxybenzylidene-2-tetrachlorophthalimido-1-thio- $\beta$-D-glucopyranoside}

(4.1).

To a solution of phenyl 2-deoxy-2-tetrachlorophthalimido-1-thio- $\beta$-D-glucopyranoside ${ }^{1}$ (3.1) (3.70 g, 6.86 mmol) in DMF $(20 \mathrm{~mL})$ was added anisaldehyde dimethyl acetal $(2.34 \mathrm{~mL}, 13.7 \mathrm{mmol})$ and $p$ toluenesulfonic acid monohydrate $(131 \mathrm{mg}, 0.689 \mathrm{mmol})$ and the reaction mixture was heated at $35{ }^{\circ} \mathrm{C}$ under reduced pressure (to remove methanol as it was generated) for $3 \mathrm{~h}$. The reaction mixture was then cooled to room temperature, quenched with solid $\mathrm{NaHCO}_{3}(500 \mathrm{mg})$, and concentrated in vacuo. The residue was redissolved in $\mathrm{CH}_{2} \mathrm{Cl}_{2}$ and washed with saturated aqueous $\mathrm{NaHCO}_{3}, \mathrm{H}_{2} \mathrm{O}$, and brine. The organic layer was dried over $\mathrm{Na}_{2} \mathrm{SO}_{4}$, decanted, and then concentrated in vacuo. Purification of the crude residue by silica gel chromatography $\left(2 \% \mathrm{EtOAc} / \mathrm{CH}_{2} \mathrm{Cl}_{2}\right)$ afforded the title compound $(4.50 \mathrm{~g}, 6.85$ mmol, 100\%). ${ }^{1} \mathrm{H}$ NMR (400 MHz, $\left.\mathrm{CDCl}_{3}\right): \delta \mathrm{ppm} 7.39-7.33$ (m, $\left.2 \mathrm{H}, \mathrm{ArH}\right), 7.29(\mathrm{~d}, J=8.4 \mathrm{~Hz}, 2 \mathrm{H}$, ArH), 7.27 - $7.22(\mathrm{~m}, 3 \mathrm{H}, \mathrm{ArH}), 6.81(\mathrm{~d}, J=8.8 \mathrm{~Hz}, 2 \mathrm{H}, \mathrm{ArH}), 5.59$ (d, J=10.6 Hz, $1 \mathrm{H}, \mathrm{H}-1), 5.44$ (s, 
1 H, CHPMP), 4.57 (ddd, J=9.6, 9.6, 3.5 Hz, $1 \mathrm{H}, \mathrm{H}-3$ ), 4.34 (dd, $J=10.4,4.9 \mathrm{~Hz}, 1 \mathrm{H}, \mathrm{H}-6$ ), 4.25 (dd, $J$ $=10.4,10.4 \mathrm{~Hz}, 1 \mathrm{H}, \mathrm{H}-2), 3.79$ (s, $3 \mathrm{H}, \mathrm{OMe}), 3.75$ (dd, $J=10.4,10.4 \mathrm{~Hz}, 1 \mathrm{H}, \mathrm{H}-6), 3.61$ (ddd, $J=9.6$, 9.6, 4.9 Hz, $1 \mathrm{H}, \mathrm{H}-5), 3.49$ (dd, $J=9.1,9.1 \mathrm{~Hz}, 1 \mathrm{H}, \mathrm{H}-4), 3.10(\mathrm{~d}, J=3.3 \mathrm{~Hz}, 1 \mathrm{H}, \mathrm{OH}) ;{ }^{13} \mathrm{C}$ NMR $(100$ $\left.\mathrm{MHz}, \mathrm{CDCl}_{3}\right): \delta \mathrm{ppm} 163.68,162.68,160.34,140.48,140.36,132.48,131.67,130.25,129.83,129.19$, 128.26, 127.49, 127.17, 127.08, 113.77, 101.84, 83.78, 81.73, 70.47, 69.24, 68.57, 56.37, 55.43; HRMS calcd for $\mathrm{C}_{28} \mathrm{H}_{22} \mathrm{Cl}_{4} \mathrm{NO}_{7} \mathrm{~S}[\mathrm{M}+\mathrm{H}]^{+}: 655.9871$, found 655.9843 .

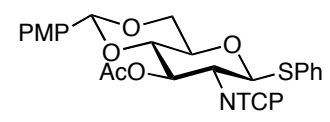

Phenyl 3-O-Acetyl-2-deoxy-4,6-O-p-methoxybenzylidene-2-tetrachlorophthalimido-1-thio- $\beta$-Dglucopyranoside (4.2).

To a solution of $4.1(3.62 \mathrm{~g}, 5.51 \mathrm{mmol})$ in pyridine $(30 \mathrm{~mL})$ was added acetic anhydride $(1.56 \mathrm{~mL}, 16.5$ mmol). After stirring overnight at room temperature, the reaction was concentrated and separated by silica gel chromatography $\left(\mathrm{CH}_{2} \mathrm{Cl}_{2}\right)$ to afford the title compound $(3.81 \mathrm{~g}, 5.45 \mathrm{mmol}, 99 \%) .{ }^{1} \mathrm{H}$ NMR $(500$ $\left.\mathrm{MHz}, \mathrm{CDCl}_{3}\right): \delta$ ppm $7.41-7.38(\mathrm{~m}, 2 \mathrm{H}, \mathrm{ArH}), 7.36(\mathrm{~d}, J=8.8 \mathrm{~Hz}, 2 \mathrm{H}, \mathrm{ArH}), 7.31-7.28(\mathrm{~m}, 3 \mathrm{H}$, ArH), 6.87 (d, $J=9.3 \mathrm{~Hz}, 2 \mathrm{H}, \mathrm{ArH}), 5.82-5.78$ (m, $1 \mathrm{H}, \mathrm{H}-3), 5.79$ (d, $J=10.7 \mathrm{~Hz}, 1 \mathrm{H}, \mathrm{H}-1), 5.49$ (s, 1 H, CHPMP), 4.41 (dd, $J=9.8,3.4 \mathrm{~Hz}, 1 \mathrm{H}, \mathrm{H}-6), 4.34$ (dd, $J=10.3,10.3 \mathrm{~Hz}, 1 \mathrm{H}, \mathrm{H}-2), 3.79$ (s, $3 \mathrm{H}$, OMe), 3.84 - 3.74 (m, 3 H, H-4,5,6), 1.90 (s, $3 \mathrm{H}, \mathrm{OAc}) ;{ }^{13} \mathrm{C} \mathrm{NMR}\left(126 \mathrm{MHz}, \mathrm{CDCl}_{3}\right.$ ): $\delta$ ppm 170.87, $163.41,162.59,160.34,140.88,140.56,133.15,130.76,130.23,130.04,129.32,129.19,128.59,127.71$, $127.25,126.90,113.73,101.82,83.14,78.64,70.83,70.67,68.55,55.39,55.20,20.72$; HRMS calcd for $\mathrm{C}_{30} \mathrm{H}_{24} \mathrm{Cl}_{4} \mathrm{NO}_{8} \mathrm{~S}[\mathrm{M}+\mathrm{H}]^{+}:$697.9976, found 697.9971.

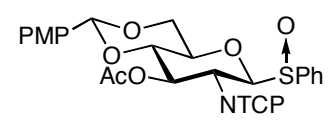

Phenyl 3-O-Acetyl-2-deoxy-4,6-O-p-methoxybenzylidene-2-tetrachlorophthalimido-1-thio- $\beta$-Dglucopyranoside $S$-Oxide (4). 
The sulfide 4 (1.57 g, $2.25 \mathrm{mmol})$ in $\mathrm{CH}_{2} \mathrm{Cl}_{2}(50 \mathrm{~mL})$ was chilled to $-78{ }^{\circ} \mathrm{C}$ and 3-chloroperoxybenzoic acid (517 $\mathrm{mg}$ of $75 \%$ dispersion, $2.25 \mathrm{mmol}$ ) was added. After $1 \mathrm{~h}$, the reaction was slowly warmed to $-30{ }^{\circ} \mathrm{C}$ then diluted with $\mathrm{CH}_{2} \mathrm{Cl}_{2}$ and extracted from saturated aqueous $\mathrm{NaHCO}_{3}$ and brine. The organic layer was dried over $\mathrm{Na}_{2} \mathrm{SO}_{4}$, decanted, and concentrated in vacuo. Purification of the crude residue by silica gel chromatography $\left(5 \% \mathrm{EtOAc} / \mathrm{CH}_{2} \mathrm{Cl}_{2}\right)$ afforded a mixture of sulfoxide diastereomers $(1.51 \mathrm{~g}$, $2.10 \mathrm{mmol}, 93 \%)$. Less polar diastereomer: $(1.29 \mathrm{~g}){ }^{1} \mathrm{H}$ NMR $\left(500 \mathrm{MHz}, \mathrm{CDCl}_{3}\right): \delta \mathrm{ppm} 7.44(\mathrm{~d}, J=8.3$ $\mathrm{Hz}, 2 \mathrm{H}, \mathrm{ArH}), 7.35$ (d, $J=8.8 \mathrm{~Hz}, 2 \mathrm{H}, \mathrm{ArH}), 7.20$ (t, $J=7.8 \mathrm{~Hz}, 2 \mathrm{H}, \mathrm{ArH}), 7.00$ (t, $J=7.3 \mathrm{~Hz}, 1 \mathrm{H}$, ArH), $6.86(\mathrm{~d}, J=8.8 \mathrm{~Hz}, 2 \mathrm{H}, \mathrm{ArH}), 5.73$ (dd, $J=9.3,9.3 \mathrm{~Hz}, 1 \mathrm{H}, \mathrm{H}-3), 5.52$ (d, $J=9.8 \mathrm{~Hz}, 1 \mathrm{H}, \mathrm{H}-1)$, 5.50 (s, $1 \mathrm{H}$, CHPMP), 4.98 (dd, $J=9.8,9.8 \mathrm{~Hz}, 1 \mathrm{H}, \mathrm{H}-2), 4.45$ - 4.38 (m, $1 \mathrm{H}, \mathrm{H}-6), 3.88$ - 3.80 (m, $3 \mathrm{H}$, $\mathrm{H}-4,5,6), 3.78$ (s, $3 \mathrm{H}, \mathrm{OMe}), 1.89$ (s, $3 \mathrm{H}, \mathrm{OAc}) ;{ }^{13} \mathrm{C} \mathrm{NMR}\left(125 \mathrm{MHz}, \mathrm{CDCl}_{3}\right.$ ): $\delta$ ppm 171.13 (s), 163.24 , $161.64,160.36,140.29,140.18,139.43,130.11,129.68,129.60,129.13,128.94,127.68,126.81,126.73$, 123.94, 113.71, 101.80, 90.12, 77.61, 71.09, 70.89, 68.13, 55.38, 47.32, 20.66. More polar diastereomer: $(0.22 \mathrm{~g}){ }^{1} \mathrm{H}$ NMR $\left(500 \mathrm{MHz}, \mathrm{CDCl}_{3}\right): \delta \mathrm{ppm} 7.59(\mathrm{~d}, J=6.8 \mathrm{~Hz}, 2 \mathrm{H}, \mathrm{ArH}), 7.54-7.47$ (m, $\left.3 \mathrm{H}, \mathrm{ArH}\right)$, $7.32(\mathrm{~d}, J=8.8 \mathrm{~Hz}, 2 \mathrm{H}, \mathrm{ArH}), 6.86(\mathrm{~d}, J=8.8 \mathrm{~Hz}, 2 \mathrm{H}, \mathrm{ArH}), 5.75$ (dd, $J=9.3,9.3 \mathrm{~Hz}, 1 \mathrm{H}, \mathrm{H}-3), 5.46$ (d, $J=10.3 \mathrm{~Hz}, 1 \mathrm{H}, \mathrm{H}-1), 5.44$ (s, $1 \mathrm{H}, \mathrm{CHPMP}), 4.69$ (dd, $J=10.0,10.0 \mathrm{~Hz}, 1 \mathrm{H}, \mathrm{H}-2), 4.23$ (dd, $J=$ 10.0, 3.2 Hz, $1 \mathrm{H}, \mathrm{H}-6), 3.78$ (s, $3 \mathrm{H}, \mathrm{OMe}$ ), 3.80 - 3.67 (m, $3 \mathrm{H}, \mathrm{H}-4,5,6), 1.90$ (s, $3 \mathrm{H}, \mathrm{OAc}$ ); ${ }^{13} \mathrm{C}$ NMR $\left(125 \mathrm{MHz}, \mathrm{CDCl}_{3}\right): \delta \mathrm{ppm} \mathrm{171.14}, 163.89,162.23,160.40,140.98,140.76,131.91,130.23,129.16$ $129.07,127.70,127.26,126.86,125.88,113.74,101.90,87.06,78.00,71.07,70.90,68.03,55.40,50.79$, 20.68; HRMS calcd for $\mathrm{C}_{30} \mathrm{H}_{27} \mathrm{Cl}_{4} \mathrm{~N}_{2} \mathrm{O}_{9} \mathrm{~S}\left[\mathrm{M}+\mathrm{NH}_{4}\right]^{+}$: 731.0191, found 731.0214.

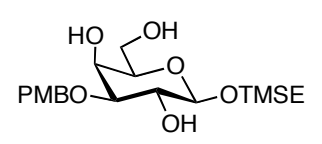

\section{2-(Trimethylsilyl)ethyl 3- $O$-p-Methoxybenzyl- $\beta$-D-galactopyranoside (5.02).}

To a suspension of 2-(trimethylsilyl)ethyl $\beta$-D-galactopyranoside ${ }^{3,4}(\mathbf{5 . 0 1})(42.3 \mathrm{~g}, 151 \mathrm{mmol})$ in $350 \mathrm{~mL}$ of methanol was added dibutyltin oxide $(56.3 \mathrm{~g}, 227 \mathrm{mmol})$ and the mixture was heated at reflux until the 
material completely dissolved $(3.5 \mathrm{~h})$. The clear reaction mixture was then concentrated and azeotroped 3x from toluene. Benzene $(500 \mathrm{~mL}), p$-methoxybenzyl chloride $(61.5 \mathrm{~mL}, 453 \mathrm{mmol})$, tetrabutylammonium bromide $(24.3 \mathrm{~g}, 75.5 \mathrm{mmol})$ and $4 \AA$ molecular sieves $(10 \mathrm{~g})$ were then added and the mixture was refluxed for $1 \mathrm{~h}$. The mixture was then cooled to $0{ }^{0} \mathrm{C}$, quenched with $30 \mathrm{~mL}$ diethylamine, filtered through a pad of celite, and the filtrate was concentrated in vacuo. The crude residue was purified by silica gel chromatography (gradient 50-66\% EtoAc/ petroleum ether) to give the title compound (59.0 g, $147 \mathrm{mmol}, 97 \%) .{ }^{1} \mathrm{H} \mathrm{NMR}\left(600 \mathrm{MHz}, \mathrm{CDCl}_{3}\right): \delta \mathrm{ppm} 7.30(\mathrm{~d}, J=8.5 \mathrm{~Hz}, 2 \mathrm{H}$, PMPH), 6.88 (d, $J=8.5 \mathrm{~Hz}, 2 \mathrm{H}, \mathrm{PMPH}), 4.67,4.65$ (ABq, $J=11.7 \mathrm{~Hz}, 1 \mathrm{H}$ each, $\left.\mathrm{OCH}_{2} \mathrm{PMP}\right), 4.25$ (d, $J$ $=7.9 \mathrm{~Hz}, 1 \mathrm{H}, \mathrm{H}-1), 4.01\left(\mathrm{ddd}, J=11.7,9.7,5.6 \mathrm{~Hz}, 1 \mathrm{H}, \mathrm{OCH} H \mathrm{CH}_{2} \mathrm{SiMe}_{3}\right), 3.98(\mathrm{~d}, J=2.9 \mathrm{~Hz}, 1 \mathrm{H}, \mathrm{H}-$ 4), $3.94(\mathrm{dd}, J=11.4,6.4 \mathrm{~Hz}, 1 \mathrm{H}, \mathrm{H}-6), 3.82$ (dd, $J=11.7,5.0 \mathrm{~Hz}, 1 \mathrm{H}, \mathrm{H}-6), 3.80$ (s, $3 \mathrm{H}, \mathrm{OMe}), 3.74$ (dd, $J=8.2,8.2 \mathrm{~Hz}, 1 \mathrm{H}, \mathrm{H}-2), 3.57$ (ddd, $J=11.3,9.6,6.0 \mathrm{~Hz}, 1 \mathrm{H}, \mathrm{OCH} H \mathrm{CH}_{2} \mathrm{SiMe}_{3}$ ), 3.47 (dd, $J=5.6$, 5.6 Hz, $1 \mathrm{H}, \mathrm{H}-5), 3.41$ (dd, $J=9.4,3.2 \mathrm{~Hz}, 1 \mathrm{H}, \mathrm{H}-3$ ), 2.74 (br s, $1 \mathrm{H}, \mathrm{OH}), 2.53$ (br s, $1 \mathrm{H}, \mathrm{OH}), 2.42$ (br s, $1 \mathrm{H}, \mathrm{OH}), 1.08-0.92\left(\mathrm{~m}, 2 \mathrm{H}, \mathrm{OCH}_{2} \mathrm{CH}_{2} \mathrm{SiMe}_{3}\right), 0.01\left(\mathrm{~s}, 9 \mathrm{H}, \mathrm{SiMe}_{3}\right) ;{ }^{13} \mathrm{C} \mathrm{NMR}\left(100 \mathrm{MHz}, \mathrm{CDCl}_{3}\right): \delta$ ppm 159.46, 129.74, 129.66, 114.00, 102.66, 79.84, 74.21, 71.62, 70.85, 67.39, 66.74, 62.02, 55.29, 18.25, -1.37; HRMS calcd for $\mathrm{C}_{19} \mathrm{H}_{36} \mathrm{NO}_{7} \mathrm{Si}\left[\mathrm{M}+\mathrm{NH}_{4}\right]^{+}: 418.2261$, found 418.2269.

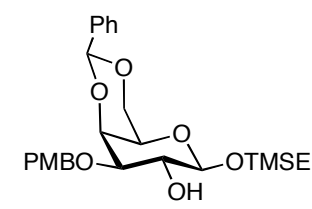

\section{2-(Trimethylsilyl)ethyl 4,6-O-Benzylidene-3- $O$-p-methoxybenzyl- $\beta$-D-galactopyranoside (5.03).}

To a solution of $\mathbf{5 . 0 2}(3.06 \mathrm{~g}, 7.64 \mathrm{mmol})$ in $10 \mathrm{~mL}$ DMF was added benzaldehyde dimethyl acetal (1.5 $\mathrm{mL}, 9.93 \mathrm{mmol})$ and $p$-toluenesulfonic acid monohydrate $(145 \mathrm{mg}, 0.76 \mathrm{mmol})$ and the reaction mixture was heated at $55{ }^{\circ} \mathrm{C}$ under reduced pressure (to remove generated methanol) for $3 \mathrm{~h}$. The reaction mixture was then cooled to room temperature, quenched with solid sodium bicarbonate $(500 \mathrm{mg})$, and concentrated in vacuo. The residue was redissolved in $\mathrm{CH}_{2} \mathrm{Cl}_{2}$ and washed with saturated aqueous $\mathrm{NaHCO}_{3}, \mathrm{H}_{2} \mathrm{O}$, and brine. The organic layer was dried over $\mathrm{Na}_{2} \mathrm{SO}_{4}$, decanted, and then concentrated in 
vacuo. The residue was purified by silica gel chromatography (30\% EtOAc/petroleum ether) to afford the title compound (3.05 g, $6.24 \mathrm{mmol}, 82 \%) .{ }^{1} \mathrm{H} \mathrm{NMR}\left(500 \mathrm{MHz}, \mathrm{CDCl}_{3}\right): \delta \mathrm{ppm} 7.53(\mathrm{dd}, J=7.6,1.7 \mathrm{~Hz}$, $2 \mathrm{H}, \mathrm{PhH}), 7.38$ - 7.30 (m, 5 H, PhH, PMPH), 6.86 (d, J=8.3 Hz, 2 H, PMPH), 5.46 (s, $1 \mathrm{H}, \mathrm{CHPh})$, 4.71, 4.67 (ABq, $J=11.7 \mathrm{~Hz}, 1 \mathrm{H}$ each, $\left.\mathrm{OCH}_{2} \mathrm{PMP}\right), 4.29$ - 4.28 (m, $\left.1 \mathrm{H}, \mathrm{H}-6\right), 4.10$ (d, $J=2.9 \mathrm{~Hz}, 1 \mathrm{H}$, H-4), 4.06 (ddd, $J=9.6,5.7,5.6 \mathrm{~Hz}, 1 \mathrm{H}, \mathrm{OCH} H \mathrm{CH}_{2} \mathrm{SiMe}_{3}$ ), 4.02 (dd, $\left.J=12.7,2.0 \mathrm{~Hz}, 1 \mathrm{H}, \mathrm{H}-6\right), 3.97$ $(\mathrm{dd}, J=9.3,9.3 \mathrm{~Hz}, 1 \mathrm{H}, \mathrm{H}-2), 3.79(\mathrm{~s}, 3 \mathrm{H}, \mathrm{OMe}), 3.58(\mathrm{ddd}, J=11.2,9.5,6.1 \mathrm{~Hz}, 1 \mathrm{H}$, $\mathrm{OCH} H C H_{2} \mathrm{SiMe}_{3}$ ), 3.47 (dd, $J=9.8,3.4 \mathrm{~Hz}, 1 \mathrm{H}, \mathrm{H}-3$ ), 3.33 (s, $\left.1 \mathrm{H}, \mathrm{H}-5\right), 2.54$ (s, $\left.1 \mathrm{H}, \mathrm{OH}\right), 1.11-0.98$ (m, $2 \mathrm{H}, \mathrm{OCH}_{2} \mathrm{CH}_{2} \mathrm{SiMe}_{3}$ ), 0.03 (s, $\left.9 \mathrm{H}, \mathrm{SiMe}_{3}\right) ;{ }^{13} \mathrm{C} \mathrm{NMR}\left(125 \mathrm{MHz}, \mathrm{CDCl}_{3}\right.$ ): $\delta \mathrm{ppm}$ 159.36, 137.90, $130.23,129.50,128.90,128.11,126.47,113.87,102.49,101.16,78.81,73.24,71.09,70.07,69.34,67.05$ 66.65, 55.31, 18.22, -1.34; HRMS calcd for $\mathrm{C}_{26} \mathrm{H}_{40} \mathrm{NO}_{7} \mathrm{Si}\left[\mathrm{M}+\mathrm{NH}_{4}\right]^{+}:$506.2574, found 506.2570.

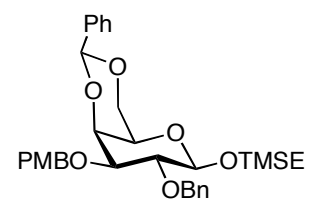

\section{2-(Trimethylsilyl)ethyl 2-O-Benzyl-4,6-O-benzylidene-3-O-p-methoxybenzyl- $\beta$-D-galactopyranoside} (5.04).

A solution of $5.03(3.05 \mathrm{~g}, 6.24 \mathrm{mmol})$ in DMF $(10 \mathrm{~mL})$ was chilled to $0{ }^{\circ} \mathrm{C}$ then $\mathrm{NaH}(315 \mathrm{mg}, 12.5$ mmol) was added. The reaction mixture was stirred for 30 minutes then treated with benzyl bromide (2.1 $\mathrm{mL}, 12.5 \mathrm{mmol})$ and stirred for an additional $1 \mathrm{~h}$ at $0{ }^{\circ} \mathrm{C}$. After being quenched with methanol $(5 \mathrm{~mL})$, the mixture was concentrated in vacuo. The crude residue was redissolved in $\mathrm{CH}_{2} \mathrm{Cl}_{2}$ and washed with saturated aqueous $\mathrm{NaHCO}_{3}, \mathrm{H}_{2} \mathrm{O}$, and brine. The organic layer was dried over $\mathrm{Na}_{2} \mathrm{SO}_{4}$, decanted, and concentrated in vacuo. The residue was purified by silica gel chromatography $(20 \%$ EtOAc/petroleum ether) to afford the title compound (2.96 g, $5.11 \mathrm{mmol}, 82 \%) .{ }^{1} \mathrm{H} \mathrm{NMR}\left(600 \mathrm{MHz}, \mathrm{CDCl}_{3}\right): \delta \mathrm{ppm} 7.57$ (d, $J=6.4 \mathrm{~Hz}, 2 \mathrm{H}, \mathrm{PhH}), 7.41$ (d, $J=7.3 \mathrm{~Hz}, 2 \mathrm{H}, \mathrm{PhH}), 7.39$ - 7.32 (m, $5 \mathrm{H}, \mathrm{PhH}), 7.32$ - 7.27 (m, $3 \mathrm{H}$, PhH, PMPH), 6.84 (d, J=8.8 Hz, 2 H, PMPH), 5.50 (s, $1 \mathrm{H}, \mathrm{CHPh}), 4.95,4.79$ (ABq, J=10.8 Hz, $1 \mathrm{H}$ each, $\left.\mathrm{OCH}_{2} \mathrm{Ph}\right), 4.72,4.69\left(\mathrm{ABq}, J=12.0 \mathrm{~Hz}, 1 \mathrm{H}\right.$ each, $\left.\mathrm{OCH}_{2} \mathrm{PMP}\right), 4.40$ (d, $\left.J=7.6 \mathrm{~Hz}, 1 \mathrm{H}, \mathrm{H}-1\right), 4.31$ 
(d, $J=12.3 \mathrm{~Hz}, 1 \mathrm{H}, \mathrm{H}-6), 4.08(\mathrm{~d}, J=3.2 \mathrm{~Hz}, 1 \mathrm{H}, \mathrm{H}-4), 4.10-4.04\left(\mathrm{~m}, 1 \mathrm{H}, \mathrm{OCH} H \mathrm{CH} \mathrm{SiMe}_{3}\right), 4.01$ (dd, $J=12.2,1.3 \mathrm{~Hz}, 1 \mathrm{H}, \mathrm{H}-6), 3.83$ (dd, $J=9.7,7.9 \mathrm{~Hz}, 1 \mathrm{H}, \mathrm{H}-2), 3.80$ (s, $3 \mathrm{H}, \mathrm{OMe}), 3.62$ - 3.56 (m, 1 $\mathrm{H}, \mathrm{OCH} H \mathrm{CH}_{2} \mathrm{SiMe}_{3}$ ), 3.54 (dd, $\left.J=9.7,3.5 \mathrm{~Hz}, 1 \mathrm{H}, \mathrm{H}-3\right), 3.31$ (br s, $\left.1 \mathrm{H}, \mathrm{H}-5\right), 1.11$ - 1.02 (m, $2 \mathrm{H}$, $\mathrm{OCH}_{2} \mathrm{CH}_{2} \mathrm{SiMe}_{3}$ ), 0.04 (s, $\left.9 \mathrm{H}, \mathrm{SiMe}_{3}\right) ;{ }^{13} \mathrm{C} \mathrm{NMR}\left(100 \mathrm{MHz}, \mathrm{CDCl}_{3}\right): \delta \mathrm{ppm} 159.30,139.16,138.03$, $130.62,129.49,129.01,128.36,128.20,128.17,127.60,126.70,113.82,103.36,101.51,78.90,78.73$, 75.36, 74.22, 71.74, 69.39, 67.47, 66.47, 55.38, 18.61, -1.27; HRMS calcd for $\mathrm{C}_{33} \mathrm{H}_{46} \mathrm{NO}_{7} \mathrm{Si}\left[\mathrm{M}+\mathrm{NH}_{4}\right]^{+}$: 596.3044, found 596.3034 .

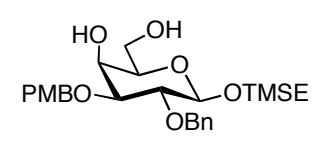

\section{2-(Trimethylsilyl)ethyl 2-O-Benzyl-3- $O$-p-methoxybenzyl- $\beta$-D-galactopyranoside (5.05).}

To a stirred suspension of $\mathbf{5 . 0 4}(3.17 \mathrm{~g}, 5.48 \mathrm{mmol})$ in methanol $(40 \mathrm{~mL})$ was added $p$-toluenesulfonic acid monohydrate $(104 \mathrm{mg}, 0.55 \mathrm{mmol})$. After stirring for $2 \mathrm{~h}$ the mixture became clear and then a portion of solid $\mathrm{NaHCO}_{3}(500 \mathrm{mg})$ was added to quench the reaction. After concentration, the residue was redissolved in $\mathrm{CH}_{2} \mathrm{Cl}_{2}$ then washed with saturated aqueous $\mathrm{NaHCO}_{3}$ and $\mathrm{H}_{2} \mathrm{O}$. The organic layer was dried over $\mathrm{Na}_{2} \mathrm{SO}_{4}$, then concentrated in vacuo. The residue was purified by silica gel chromatography (40\% EtOAc/petroleum ether) to afford the title compound (2.42 g, $6.24 \mathrm{mmol}, 90 \%) .{ }^{1} \mathrm{H}$ NMR (600 $\left.\mathrm{MHz}, \mathrm{CDCl}_{3}\right): \delta$ ppm $7.38(\mathrm{~d}, J=7.3 \mathrm{~Hz}, 2 \mathrm{H}, \mathrm{PhH}), 7.34(\mathrm{t}, J=7.5 \mathrm{~Hz}, 2 \mathrm{H}, \mathrm{PhH}), 7.29$ (t, $J=7.3 \mathrm{~Hz}, 1$ H, PhH), 7.25 (d, $J=8.5 \mathrm{~Hz}, 2 \mathrm{H}, \mathrm{PMPH}), 6.85$ (d, $J=8.2 \mathrm{~Hz}, 2 \mathrm{H}, \mathrm{PMPH}), 4.93,4.73$ (ABq, $J=11.0$ $\mathrm{Hz}, 1 \mathrm{H}$ each, $\left.\mathrm{OCH}_{2} \mathrm{Ph}\right), 4.66,4.64$ (ABq, $J=11.2 \mathrm{~Hz}, 1 \mathrm{H}$ each, $\left.\mathrm{OCH}_{2} \mathrm{PMP}\right), 4.38$ (d, $J=7.6 \mathrm{~Hz}, 1 \mathrm{H}, \mathrm{H}-$ 1), 4.06 - 4.00 (m, 1H, OCHHCH $\mathrm{SiMe}_{3}$ ), 4.02 - 3.94 (m, $\left.2 \mathrm{H}, \mathrm{H}-4,6\right), 3.83$ (dd, J = 8.6, 4.5 Hz, $1 \mathrm{H}, \mathrm{H}-$ 6), 3.80 (s, $3 \mathrm{H}, \mathrm{OMe}$ ), 3.64 - 3.57 (m, $2 \mathrm{H}, \mathrm{H}-2, \mathrm{OCH} H C H_{2} \mathrm{SiMe}_{3}$ ), 3.49 (dd, J= 9.4, $3.5 \mathrm{~Hz}, 1 \mathrm{H}, \mathrm{H}-3$ ), $3.45(\mathrm{t}, J=5.4 \mathrm{~Hz}, 1 \mathrm{H}, \mathrm{H}-5), 2.64(\mathrm{~s}, 1 \mathrm{H}, \mathrm{OH}), 2.20(\mathrm{dd}, J=8.5,4.1 \mathrm{~Hz}, 1 \mathrm{H}, \mathrm{OH}), 1.04$ (t, $J=8.5 \mathrm{~Hz}, 2$ $\mathrm{H}, \mathrm{OCH}_{2} \mathrm{CH}_{2} \mathrm{SiMe}_{3}$ ), 0.02 (s, $\left.9 \mathrm{H}, \mathrm{SiMe}_{3}\right) ;{ }^{13} \mathrm{C} \mathrm{NMR}\left(100 \mathrm{MHz}, \mathrm{CDCl}_{3}\right.$ ): $\delta$ ppm 159.52, 138.82, 129.97, $129.65,128.42,128.17,127.73,114.01,103.43,80.17,79.13,75.31,73.98,72.30,67.67,67.60,62.72$, 55.40, 18.68, -1.29; HRMS calcd for $\mathrm{C}_{26} \mathrm{H}_{42} \mathrm{NO}_{7} \mathrm{Si}\left[\mathrm{M}+\mathrm{NH}_{4}\right]^{+}$: 508.2731, found 508.2715. 


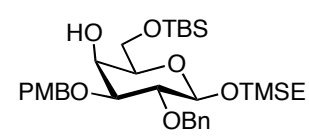

2-(Trimethylsilyl)ethyl 2-O-Benzyl-6-O-tert-butyldimethylsilyl-3-O-p-methoxybenzyl- $\beta$-Dgalactopyranoside (5.06).

A solution of $\mathbf{5 . 0 5}(8.90 \mathrm{~g}, 18 \mathrm{mmol})$ in DMF $(60 \mathrm{~mL})$ was treated with tert-butyldimethylsilyl chloride $(6.8 \mathrm{~g}, 45.2 \mathrm{mmol})$ and imidazole $(3.1 \mathrm{~g}, 45.2 \mathrm{mmol})$. After stirring for $8 \mathrm{~h}$, the reaction was concentrated in vacuo. The residue was redissolved in $\mathrm{CH}_{2} \mathrm{Cl}_{2}$ then washed with $\mathrm{H}_{2} \mathrm{O}$. The organic layer was dried over $\mathrm{Na}_{2} \mathrm{SO}_{4}$, decanted, and concentrated in vacuo. The residue was purified by silica gel chromatography (10\% EtOAc/petroleum ether) to afford the title compound (10.5 g, $17.4 \mathrm{mmol}, 96 \%)$. ${ }^{1} \mathrm{H}$ NMR (600 MHz, CDCl $)$ ): $\delta$ ppm 7.38 (d, $\left.J=7.0 \mathrm{~Hz}, 2 \mathrm{H}, \mathrm{PhH}\right), 7.33$ (t, $\left.J=7.6 \mathrm{~Hz}, 2 \mathrm{H}, \mathrm{PhH}\right), 7.30$ 7.26 (m, 3 H, PhH, PMPH), 6.85 (d, $J=8.2 \mathrm{~Hz}, 2 \mathrm{H}, \mathrm{PMPH}), 4.92,4.73(\mathrm{ABq}, J=11.1 \mathrm{~Hz}, 1 \mathrm{H}$ each, $\left.\mathrm{OCH}_{2} \mathrm{Ph}\right), 4.68,4.64\left(\mathrm{ABq}, J=11.4 \mathrm{~Hz}, 1 \mathrm{H}\right.$ each, $\left.\mathrm{OCH}_{2} \mathrm{PMP}\right), 4.34(\mathrm{~d}, J=7.9 \mathrm{~Hz}, 1 \mathrm{H}, \mathrm{H}-1), 4.04-$ $3.98\left(\mathrm{~m}, 1 \mathrm{H}, \mathrm{OCH} H \mathrm{CH}_{2} \mathrm{SiMe}_{3}\right), 3.94$ (br s, $\left.1 \mathrm{H}, \mathrm{H}-4\right), 3.89$ (dd, $\left.J=10.3,6.2 \mathrm{~Hz}, 1 \mathrm{H}, \mathrm{H}-6\right), 3.82$ (dd, $J=$ 10.4, $5.7 \mathrm{~Hz}, 1 \mathrm{H}, \mathrm{H}-6), 3.80$ (s, $3 \mathrm{H}, \mathrm{OMe}), 3.64-3.54$ (m, $\left.2 \mathrm{H}, \mathrm{H}-2, \mathrm{OCH} H \mathrm{CH}_{2} \mathrm{SiMe}_{3}\right), 3.45$ (dd, $J=$ 9.4, 3.2 Hz, $1 \mathrm{H}, \mathrm{H}-3), 3.38(\mathrm{t}, J=6.0 \mathrm{~Hz}, 1 \mathrm{H}, \mathrm{H}-5), 2.50($ br s, $1 \mathrm{H}, \mathrm{OH}), 1.03(\mathrm{t}, J=8.5 \mathrm{~Hz}, 2 \mathrm{H}$, $\mathrm{OCH}_{2} \mathrm{CH}_{2} \mathrm{SiMe}_{3}$ ), 0.90 (s, $9 \mathrm{H}, \mathrm{SiCMe}_{3}$ ), 0.082, 0.078 (s, $3 \mathrm{H}$ each, $\mathrm{SiMe}_{2}$ ), 0.02 (s, $\left.9 \mathrm{H}, \mathrm{SiMe}_{3}\right) ;{ }^{13} \mathrm{C}$ NMR (125 MHz, $\left.\mathrm{CDCl}_{3}\right)$ : $\delta$ ppm 159.41, 138.99, 130.26, 129.56, 128.35, 128.14, 127.62, 113.93, 103.33, $80.55,79.34,75.23,74.56,72.18,67.24,66.75,62.50,55.33,25.97,18.59,18.41,-1.32,-5.23,-5.28$; HRMS calcd for $\mathrm{C}_{32} \mathrm{H}_{56} \mathrm{NO}_{7} \mathrm{Si}_{2}\left[\mathrm{M}+\mathrm{NH}_{4}\right]^{+}: 622.3595$, found 622.3580 .

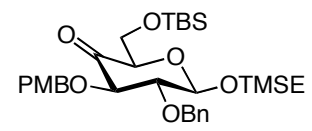

2-(Trimethylsilyl)ethyl 2-O-Benzyl-6- $O$-tert-butyldimethylsilyl-3- $O$-p-methoxybenzyl- $\beta$-D-xylohexopyranoside-4-ulose (5.07). 
Compound $5.06(10.5 \mathrm{~g}, 17.4 \mathrm{mmol})$ in DMSO $(80 \mathrm{~mL})$ and acetic anhydride $(60 \mathrm{~mL})$ was heated to $65{ }^{\circ} \mathrm{C}$ for $3 \mathrm{~h}$ then cooled to room temperature. The reaction mixture was poured into a separatory funnel, diluted with ethyl ether, and washed with $\mathrm{H}_{2} \mathrm{O}$. The aqueous layer was back-extracted with additional ethyl ether $(5 \mathrm{x})$. The organic layers were combined, dried over $\mathrm{Na}_{2} \mathrm{SO}_{4}$, and concentrated in vacuo. The resulting oil was purified by silica gel column chromatography ( $8 \%$ EtOAc/petroleum ether) to afford the title compound $(10.4 \mathrm{~g}, 17.4 \mathrm{mmol}, 100 \%) .{ }^{1} \mathrm{H} \mathrm{NMR}\left(500 \mathrm{MHz}, \mathrm{CDCl}_{3}\right): \delta \mathrm{ppm} 7.40-7.34$ (m, $6 \mathrm{H}), 7.32(\mathrm{~d}, J=8.3 \mathrm{~Hz}, 2 \mathrm{H}), 6.85(\mathrm{~d}, J=8.3 \mathrm{~Hz}, 2 \mathrm{H}), 4.88(\mathrm{~d}, J=7.8 \mathrm{~Hz}, 1 \mathrm{H}), 4.86(\mathrm{~d}, J=7.8$ $\mathrm{Hz}, 1 \mathrm{H}), 4.79-4.74(\mathrm{~m}, 2 \mathrm{H}), 4.58(\mathrm{~d}, J=11.2 \mathrm{~Hz}, 1 \mathrm{H}), 4.15-4.08(\mathrm{~m}, 3 \mathrm{H}), 4.05(\mathrm{t}, J=8.5 \mathrm{~Hz}, 1 \mathrm{H})$, $3.98(\mathrm{dd}, J=7.1,3.7 \mathrm{~Hz}, 1 \mathrm{H}), 3.85-3.81(\mathrm{~m}, 1 \mathrm{H}), 3.80(\mathrm{~s}, 3 \mathrm{H}), 3.74-3.61(\mathrm{~m}, 2 \mathrm{H}), 1.09-1.02(\mathrm{~m}, 2$ $\mathrm{H}), 0.93(\mathrm{~s}, 9 \mathrm{H}), 0.11(\mathrm{~s}, 6 \mathrm{H}), 0.06(\mathrm{~s}, 9 \mathrm{H}) ;{ }^{13} \mathrm{C} \mathrm{NMR}\left(125 \mathrm{MHz}, \mathrm{CDCl}_{3}\right): \delta \mathrm{ppm} 202.00,159.50$, $138.34,129.89,129.72,128.41,128.12,127.81,113.86,102.52,83.74,83.10,74.75,73.50,67.53,61.74$, 55.31, 25.97, 18.46, 18.42, -1.29, -5.17, -5.25; HRMS calcd for $\mathrm{C}_{32} \mathrm{H}_{54} \mathrm{NO}_{7} \mathrm{Si}_{2}\left[\mathrm{M}+\mathrm{NH}_{4}\right]^{+}: 620.3439$, found 620.3423 .

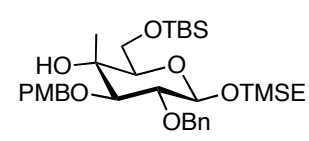

2-(Trimethylsilyl)ethyl 2-O-Benzyl-6-O-tert-butyldimethylsilyl-3- $O$-p-methoxybenzyl-4-C-methyl- $\beta$ D-glucopyranoside (5.08).

A stirred solution of $\mathbf{5 . 0 7}(9.2 \mathrm{~g}, 15 \mathrm{mmol})$ in ethyl ether $(250 \mathrm{~mL})$ was chilled to $-78{ }^{\circ} \mathrm{C}$ then methyl lithium (48 $\mathrm{mL}$ of $1.6 \mathrm{M}$ solution in diethyl ether, $76 \mathrm{mmol}$ ) was added dropwise over 20 minutes. The reaction was kept at $-78{ }^{\circ} \mathrm{C}$ for an additional $4 \mathrm{~h}$ then warmed to room temperature and carefully quenched with saturated $\mathrm{NH}_{4} \mathrm{Cl}$ and transferred to a separatory funnel containing diethyl ether. The organic phase collected then washed with $\mathrm{H}_{2} \mathrm{O}$ and brine, dried over over $\mathrm{Na}_{2} \mathrm{SO}_{4}$, decanted, and concentrated in vacuo. The resulting oil was purified by silica gel chromatography (8-12\% EtOAc/petroleum ether) to give the desired glucopyranoside product $\mathbf{5 . 0 8}(7.2 \mathrm{~g}, 11.6 \mathrm{mmol}, 76 \%)$ and its galactopyranoside diastereomer $(2.1 \mathrm{~g}, 3.4 \mathrm{mmol}, 22 \%)$. Desired glucopyranoside product 5.08: ${ }^{1} \mathrm{H}$ 
NMR (500 MHz, $\mathrm{CDCl}_{3}$ ): $\delta$ ppm $7.38-7.27$ (m, $\left.7 \mathrm{H}, \mathrm{ArH}\right), 6.86(\mathrm{~d}, J=8.8 \mathrm{~Hz}, 2 \mathrm{H}, \mathrm{PMPH}), 4.89,4.71$ $\left(\mathrm{ABq}, J=10.7 \mathrm{~Hz}, 1 \mathrm{H}\right.$ each, $\left.\mathrm{OCH}_{2} \mathrm{Ar}\right), 4.84,4.79\left(\mathrm{ABq}, J=11.0 \mathrm{~Hz}, 1 \mathrm{H}\right.$ each, $\left.\mathrm{OCH}_{2} \mathrm{Ar}\right), 4.44(\mathrm{~d}, J=$ $7.8 \mathrm{~Hz}, 1 \mathrm{H}, \mathrm{H}-1), 4.04$ - 3.97 (m, 1 H, OCH $\mathrm{HCH}_{2} \mathrm{SiMe}_{3}$ ), 3.92 - 3.81 (m, $\left.2 \mathrm{H}, \mathrm{H}-6\right), 3.80$ (s, $3 \mathrm{H}, \mathrm{OMe}$ ), 3.57 - 3.65 (m, $1 \mathrm{H}, \mathrm{OCHHCH}_{2} \mathrm{SiMe}_{3}$ ), 3.49 (d, $\left.J=9.8 \mathrm{~Hz}, 1 \mathrm{H}, \mathrm{H}-3\right), 3.42$ (t, $\left.J=6.8 \mathrm{~Hz}, 1 \mathrm{H}, \mathrm{H}-5\right), 3.27$ (dd, $J=9.8,7.8 \mathrm{~Hz}, 1 \mathrm{H}, \mathrm{H}-2), 1.30$ (s, $3 \mathrm{H}, \mathrm{Me}_{2}$ ), 1.05 (t, $J=8.3 \mathrm{~Hz}, 2 \mathrm{H}, \mathrm{OCH}_{2} \mathrm{CH}_{2} \mathrm{SiMe}_{3}$ ), 0.93 (s, $9 \mathrm{H}$,

$\mathrm{SiCMe}_{3}$ ), 0.13, 0.12 (s, $3 \mathrm{H}$ each, SiMe), 0.04 (s, $\left.9 \mathrm{H}, \mathrm{SiMe}_{3}\right) ;{ }^{13} \mathrm{C} \mathrm{NMR}\left(125 \mathrm{MHz}, \mathrm{CDCl}_{3}\right): \delta \mathrm{ppm}$ $159.21,138.96,131.46,129.60,128.41,128.14,127.66,113.85,103.85,86.30,81.41,75.78,75.32$, $75.16,75.10,67.85,62.73,55.36,25.99,18.71,18.31,16.37,-1.29,-5.32,-5.48$; HRMS calcd for $\mathrm{C}_{33} \mathrm{H}_{58} \mathrm{NO}_{7} \mathrm{Si}_{2}\left[\mathrm{M}+\mathrm{NH}_{4}\right]^{+}:$636.3752, found 636.3726 .

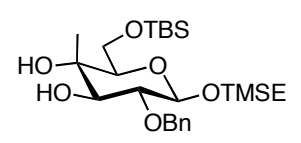

\section{2-(Trimethylsilyl)ethyl 2-O-Benzyl-6-O-tert-butyldimethylsilyl-4-C-methyl- $\beta$-D-glucopyranoside} (5.09).

To a stirred solution of $\mathbf{5 . 0 8}(7.2 \mathrm{~g}, 11.6 \mathrm{mmol})$ in $\mathrm{CH}_{2} \mathrm{Cl}_{2}(137 \mathrm{~mL})$ and $\mathrm{H}_{2} \mathrm{O}(8 \mathrm{~mL})$ at $0{ }^{\circ} \mathrm{C}$ was added 2,3-dichloro-5,6-dicyano-1,4-benzoquinone (3.4 g, $15.0 \mathrm{mmol})$. The reaction mixture was stirred for $1 \mathrm{~h}$ then diluted with $\mathrm{CH}_{2} \mathrm{Cl}_{2}$ and sequentially washed with saturated aqueous $\mathrm{NaHCO}_{3}, \mathrm{H}_{2} \mathrm{O}$, and brine. After concentration of the organic phase in vacuo, the crude residue was purified by silica gel chromatography (25\% EtOAc/petroleum ether) to afford the title compound (4.5 g, $9.0 \mathrm{mmol}, 78 \%) .{ }^{1} \mathrm{H}$ NMR (500 MHz, $\left.\mathrm{CDCl}_{3}\right): \delta$ ppm $7.39-7.32(\mathrm{~m}, 4 \mathrm{H}, \mathrm{PhH}), 7.29$ (t, $\left.J=6.8 \mathrm{~Hz}, 1 \mathrm{H}, \mathrm{PhH}\right), 4.95,4.68$ $\left(\mathrm{ABq}, J=11.2 \mathrm{~Hz}, 1 \mathrm{H}\right.$ each, $\left.\mathrm{OCH}_{2} \mathrm{Ph}\right), 4.42(\mathrm{~d}, J=7.8 \mathrm{~Hz}, 1 \mathrm{H}, \mathrm{H}-1), 4.01-3.94(\mathrm{~m}, 1 \mathrm{H}$, $\left.\mathrm{OCH} H C H_{2} \mathrm{SiMe}_{3}\right), 3.90-3.80$ (m, $\left.2 \mathrm{H}, \mathrm{H}-6\right), 3.60$ (d, J=9.8 Hz, $\left.1 \mathrm{H}, \mathrm{H}-3\right), 3.60$ - 3.54 (m, $1 \mathrm{H}$, $\mathrm{OCH} H C H_{2} \mathrm{SiMe}_{3}$ ), 3.51 (br s, $\left.1 \mathrm{H}, \mathrm{OH}\right), 3.41$ (t, $\left.J=6.8 \mathrm{~Hz}, 1 \mathrm{H}, \mathrm{H}-5\right), 3.16$ (dd, $J=9.8,7.8 \mathrm{~Hz}, 1 \mathrm{H}, \mathrm{H}-$ 3), 2.48 (br s, $1 \mathrm{H}, \mathrm{OH}), 1.25$ (s, $3 \mathrm{H}, \mathrm{Me}), 1.05$ - 0.99 (m, $2 \mathrm{H}, \mathrm{OCH}_{2} \mathrm{CH}_{2} \mathrm{SiMe}_{3}$ ), 0.90 (s, $9 \mathrm{H}, \mathrm{SiCMe}_{3}$ ), 0.11, 0.11 (s, $3 \mathrm{H}$ each, $\mathrm{SiMe}_{2}$ ), 0.02 (s, $\left.9 \mathrm{H}, \mathrm{SiMe}_{3}\right) ;{ }^{13} \mathrm{C} \mathrm{NMR}\left(125 \mathrm{MHz}, \mathrm{CDCl}_{3}\right): \delta \mathrm{ppm} \mathrm{138.73,}$ 
$128.56,128.03,127.86,103.70,80.62,78.29,75.77,74.63,73.80,67.81,62.57,25.91,18.68,18.21$, 15.31, -1.31, -5.39, -5.52; HRMS calcd for $\mathrm{C}_{25} \mathrm{H}_{50} \mathrm{NO}_{6} \mathrm{Si}_{2}\left[\mathrm{M}+\mathrm{NH}_{4}\right]^{+}:$516.3177, found 516.3165.

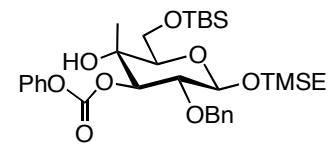

2-(Trimethylsilyl)ethyl 2-O-Benzyl-6-O-tert-butyldimethylsilyl-4-C-methyl-3-O-phenoxycarbonyl- $\beta$ D-glucopyranoside (5.10).

Compound 5.09 (4.0 g, $8.0 \mathrm{mmol})$ was dissolved in $\mathrm{CH}_{2} \mathrm{Cl}_{2}(80 \mathrm{~mL})$ and pyridine $(2.6 \mathrm{~mL}, 32 \mathrm{mmol})$ then chilled to $0{ }^{\circ} \mathrm{C}$. Phenyl chloroformate $(1.1 \mathrm{~mL}, 8.8 \mathrm{mmol})$ was added and the reaction was stirred for 20 min then quenched with $20 \mathrm{~mL} \mathrm{MeOH}$ and concentrated in vacuo. The residue was dissolved in $\mathrm{CH}_{2} \mathrm{Cl}_{2}$ then washed with $\mathrm{H}_{2} \mathrm{O}$ and brine. The organic layer was concentrated in vacuo and the residue was purified by silica gel chromatography (10\% EtOAc/petroleum ether) to afford the title compound (5.0 g, $8.0 \mathrm{mmol}, 100 \%) .{ }^{1} \mathrm{H}$ NMR (500 MHz, $\left.\mathrm{CDCl}_{3}\right): \delta \mathrm{ppm} 7.36-7.25(\mathrm{~m}, 7 \mathrm{H}, \mathrm{PhH}), 7.22(\mathrm{t}, J=7.3 \mathrm{~Hz}, 1$ H, PhH), 7.10 (d, $J=8.8 \mathrm{~Hz}, 2 \mathrm{H}, \mathrm{PhH}), 4.97$ (d, $J=10.3 \mathrm{~Hz}, 1 \mathrm{H}, \mathrm{H}-3), 4.95,4.67$ (ABq, $J=11.2 \mathrm{~Hz}$, $\mathrm{OCH}_{2} \mathrm{Ph} 1 \mathrm{H}$ each, $), 4.53(\mathrm{~d}, J=7.8 \mathrm{~Hz}, 1 \mathrm{H}, \mathrm{H}-1), 4.04-3.96\left(\mathrm{~m}, 1 \mathrm{H}, \mathrm{OCH} H \mathrm{CH}_{2} \mathrm{SiMe}_{3}\right), 3.89(\mathrm{~d}, J=$ $7.3 \mathrm{~Hz}, 2 \mathrm{H}, \mathrm{H}-6), 3.64$ (s, $1 \mathrm{H}, \mathrm{OH}), 3.63-3.58$ (m, $1 \mathrm{H}, \mathrm{OCHHCH}_{2} \mathrm{SiMe}_{3}$ ), 3.54 (t, J = 7.1 Hz, $1 \mathrm{H}, \mathrm{H}-$ 5), $3.38(\mathrm{dd}, J=10.3,7.8 \mathrm{~Hz}, 1 \mathrm{H}, \mathrm{H}-2), 1.36(\mathrm{~s}, 3 \mathrm{H}, \mathrm{Me}), 1.04\left(\mathrm{t}, J=8.8 \mathrm{~Hz}, 2 \mathrm{H}, \mathrm{OCH}_{2} \mathrm{CH}_{2} \mathrm{SiMe}_{3}\right)$, 0.91 (s, $\left.9 \mathrm{H}, \mathrm{SiCMe}_{3}\right), 0.13,0.12$ (s, $3 \mathrm{H}$ each, $\mathrm{SiMe}_{2}$ ), 0.04 (s, $\left.9 \mathrm{H}, \mathrm{SiMe}_{3}\right) ;{ }^{13} \mathrm{C} \mathrm{NMR}(125 \mathrm{MHz}$, $\left.\mathrm{CDCl}_{3}\right): \delta$ ppm 153.72, 151.34, 138.51, 129.43, 128.44, 127.66, 127.64, 125.97, 121.18, 103.63, 83.26, $79.14,75.22,74.80,73.57,68.06,62.56,25.94,18.69,18.26,16.13,-1.29,-5.38,-5.55$; HRMS calcd for $\mathrm{C}_{32} \mathrm{H}_{54} \mathrm{NO}_{8} \mathrm{Si}_{2}\left[\mathrm{M}+\mathrm{NH}_{4}\right]^{+}:$618.3044, found 618.9056.

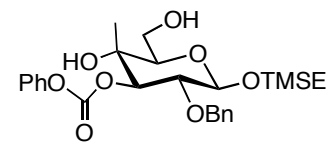

2-(Trimethylsilyl)ethyl 2-O-Benzyl-4-C-methyl-3- $O$-phenoxycarbonyl- $\beta$-D-glucopyranoside (5.11). 
To a stirred solution of $\mathbf{5 . 1 0}(4.7 \mathrm{~g}, 7.6 \mathrm{mmol})$ in $\mathrm{CH}_{3} \mathrm{CN}(30 \mathrm{~mL})$ and $\mathrm{H}_{2} \mathrm{O}(30 \mathrm{~mL})$ was added $p$ toluenesulfonic acid monohydrate $(115 \mathrm{mg}, 0.76 \mathrm{mmol})$. The reaction was stirred for $3 \mathrm{~h}$ then diluted with EtOAc and washed sequentially with saturated aqueous $\mathrm{NaHCO}_{3}, \mathrm{H}_{2} \mathrm{O}$, and brine. After concentration of the organic phase in vacuo, the crude residue was purified by silica gel chromatography (50\% EtOAc/petroleum ether) to afford the title compound (3.7 g, $7.3 \mathrm{mmol}, 96 \%) .{ }^{1} \mathrm{H} \mathrm{NMR}(500 \mathrm{MHz}$, $\left.\mathrm{CDCl}_{3}\right): \delta$ ppm $7.39-7.22(\mathrm{~m}, 8 \mathrm{H}, \mathrm{PhH}), 7.10(\mathrm{~d}, J=7.3 \mathrm{~Hz}, 2 \mathrm{H}, \mathrm{PhH}), 4.94,4.67$ (ABq, $J=11.2 \mathrm{~Hz}, 1$ $\mathrm{H}$ each, $\left.\mathrm{OCH}_{2} \mathrm{Ph}\right), 4.88(\mathrm{~d}, J=9.8 \mathrm{~Hz}, 1 \mathrm{H}, \mathrm{H}-3), 4.53(\mathrm{~d}, J=7.8 \mathrm{~Hz}, 1 \mathrm{H}, \mathrm{H}-1), 4.04-3.96$ (m, $1 \mathrm{H}$, $\mathrm{OCH} H C H_{2} \mathrm{SiMe}_{3}$ ), 3.91 (dd, $\left.J=11.7,4.9 \mathrm{~Hz}, 1 \mathrm{H}, \mathrm{H}-6\right), 3.78$ (dd, $\left.J=11.7,6.8 \mathrm{~Hz}, 1 \mathrm{H}, \mathrm{H}-6\right), 3.67$ - 3.59 (m, $\left.1 \mathrm{H}, \mathrm{OCH} H \mathrm{CH}_{2} \mathrm{SiMe}_{3}\right), 3.46$ (dd, $\left.J=6.8,5.4 \mathrm{~Hz}, 1 \mathrm{H}, \mathrm{H}-5\right), 3.39$ (dd, $\left.J=10.0,7.6 \mathrm{~Hz}, 1 \mathrm{H}, \mathrm{H}-2\right)$, $1.29(\mathrm{~s}, 3 \mathrm{H}, \mathrm{Me}), 1.04$ (t, $\left.J=8.5 \mathrm{~Hz}, 2 \mathrm{H}, \mathrm{OCH}_{2} \mathrm{CH}_{2} \mathrm{SiMe}_{3}\right), 0.04$ (s, $\left.9 \mathrm{H}, \mathrm{SiMe}_{3}\right) ;{ }^{13} \mathrm{C} \mathrm{NMR}(125 \mathrm{MHz}$, $\left.\mathrm{CDCl}_{3}\right): \delta$ ppm 154.43, 151.15, 138.34, 129.61, 128.50, 127.81, 127.73, 126.31, 121.06, 103.64, 84.03, 79.21, 77.45, 74.92, 73.40, 68.21, 61.05, 18.73, 15.90, -1.27; HRMS calcd for $\mathrm{C}_{26} \mathrm{H}_{40} \mathrm{NO}_{8} \mathrm{Si}\left[\mathrm{M}+\mathrm{NH}_{4}\right]^{+}$: 522.2523, found 522.2501.

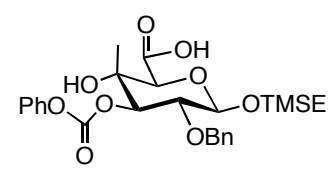

2-(Trimethylsilyl)ethyl 2- $O$-Benzyl-4-C-methyl-3- $O$-phenoxycarbonyl- $\beta$-D-glucopyranuronic acid (5.12).

Compound $5.11(3.6 \mathrm{~g}, 7.1 \mathrm{mmol})$ was dissolved in acetone $(50 \mathrm{~mL})$ then treated with a solution of potassium dichromate $(4.2 \mathrm{~g}, 14.2 \mathrm{mmol})$ in $3 \mathrm{M} \mathrm{H}_{2} \mathrm{SO}_{4}(20 \mathrm{~mL})$. The reaction mixture was heated at $70{ }^{\circ} \mathrm{C}$ for $1 \mathrm{~h}$ then cooled to room temperature. Dilution with EtOAc was followed by washing with $\mathrm{H}_{2} \mathrm{O}$. The aqueous phase was back-extracted with EtOAc $(3 \mathrm{x})$ and the combined organic extracts were washed with $\mathrm{H}_{2} \mathrm{O}$. The aqueous layer was back-extracted with EtOAc then the organic phases were combined, dried over $\mathrm{Na}_{2} \mathrm{SO}_{4}$, and concentrated in vacuo to afford the crude acid (3.7 g, $\left.7.1 \mathrm{mmol}\right)$, which was utilized in the next reaction without further purification. ${ }^{1} \mathrm{H}$ NMR $\left(500 \mathrm{MHz}, \mathrm{CDCl}_{3}\right): \delta \mathrm{ppm} 7.38-7.27$ 
(m, 7 H, PhH), 7.24 (t, J=7.3 Hz, $1 \mathrm{H}, \mathrm{PhH}), 7.09$ (d, J=7.8 Hz, $2 \mathrm{H}, \mathrm{PhH}), 5.05$ (d, J=9.8 Hz, $1 \mathrm{H}, \mathrm{H}-$ 3), 4.94, $4.68\left(\mathrm{ABq}, J=11.5 \mathrm{~Hz}, 1 \mathrm{H}\right.$ each, $\left.\mathrm{OCH}_{2} \mathrm{Ph}\right), 4.60$ (d, J= 7.8 Hz, $\left.1 \mathrm{H}, \mathrm{H}-1\right), 4.05$ (s, $\left.1 \mathrm{H}, \mathrm{H}-5\right)$, 4.09 - $4.02\left(\mathrm{~m}, 1 \mathrm{H}, \mathrm{OCH} H C H_{2} \mathrm{SiMe}_{3}\right), 3.71-3.62$ (m, $\left.1 \mathrm{H}, \mathrm{OCH} H C H_{2} \mathrm{SiMe}_{3}\right), 3.44$ (dd, $J=10.3,7.8$ $\mathrm{Hz}, 1 \mathrm{H}, \mathrm{H}-2), 1.36$ (s, $3 \mathrm{H}, \mathrm{Me}), 1.06$ (t, $\left.J=8.5 \mathrm{~Hz}, 2 \mathrm{H}, \mathrm{OCH}_{2} \mathrm{CH}_{2} \mathrm{SiMe}_{3}\right), 0.05$ (s, $\left.9 \mathrm{H}, \mathrm{SiMe}_{3}\right) ;{ }^{13} \mathrm{C}$ NMR (125 MHz, $\left.\mathrm{CDCl}_{3}\right): \delta$ ppm 170.22, 153.72, 151.18, 138.01, 129.57, 128.53, 127.92, 127.75, 126.26, $121.06,103.45,81.87,78.50,77.41,76.91,75.83,75.00,72.96,68.80,18.66,16.93,-1.27$; HRMS calcd for $\mathrm{C}_{26} \mathrm{H}_{38} \mathrm{NO}_{9} \mathrm{Si}\left[\mathrm{M}+\mathrm{NH}_{4}\right]^{+}: 536.2316$, found 536.2292.

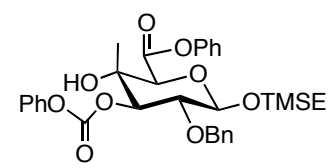

\section{Phenyl [2-(Trimethylsilyl)ethyl 2-O-benzyl-4-C-methyl-3- $O$-phenoxycarbonyl- $\beta$-D-glucopyranosid]} uronate (5.13).

The acid 5.12 (555 mg, $1.07 \mathrm{mmol})$ was dissolved in $\mathrm{CH}_{2} \mathrm{Cl}_{2}(12 \mathrm{~mL})$ and the mixture was cooled to $0{ }^{\circ} \mathrm{C}$. Phenol (504 mg, $5.35 \mathrm{mmol})$ and 4-dimethylaminopyridine (13 $\mathrm{mg}, 0.11 \mathrm{mmol})$ were added followed by $\mathrm{N}$-(3-dimethylaminopropyl)-N'-ethylcarbodiimide hydrochloride (246 mg, $1.28 \mathrm{mmol}$ ). After being stirred overnight, the reaction mixture was diluted with $\mathrm{CH}_{2} \mathrm{Cl}_{2}$ and washed with $\mathrm{H}_{2} \mathrm{O}$ and brine. The organic phase was then dried over $\mathrm{Na}_{2} \mathrm{SO}_{4}$ followed by concentration in vacuo. The crude residue was then purified by silica gel chromatography $(20 \%$ EtOAc/petroleum ether) to afford the title compound (489 mg, $0.822 \mathrm{mmol}, 77 \%) .{ }^{1} \mathrm{H} \mathrm{NMR}\left(600 \mathrm{MHz}, \mathrm{CDCl}_{3}\right)$ : $\delta \mathrm{ppm} 7.42$ (t, $\left.J=7.8 \mathrm{~Hz}, 2 \mathrm{H}, \mathrm{PhH}\right), 7.37$ (d, $J=6.7 \mathrm{~Hz}, 2 \mathrm{H}, \mathrm{PhH}), 7.36$ - 7.27 (m, $6 \mathrm{H}, \mathrm{PhH}), 7.22$ (t, $J=7.5 \mathrm{~Hz}, 1 \mathrm{H}, \mathrm{PhH}), 7.14$ (d, J= 7.6 Hz, $2 \mathrm{H}$, $\mathrm{PhH}), 7.13(\mathrm{~d}, J=7.6 \mathrm{~Hz}, 2 \mathrm{H}, \mathrm{PhH}), 5.12$ (d, $J=10.0 \mathrm{~Hz}, 1 \mathrm{H}, \mathrm{H}-3), 4.99,4.72(\mathrm{ABq}, J=11.4 \mathrm{~Hz}, 1 \mathrm{H}$ each, $\left.\mathrm{OCH}_{2} \mathrm{Ph}\right), 4.64$ (d, $\left.J=7.9 \mathrm{~Hz}, 1 \mathrm{H}, \mathrm{H}-1\right), 4.25$ (s, $\left.1 \mathrm{H}, \mathrm{H}-5\right), 4.11$ (ddd, $J=10.2,10.2,6.6 \mathrm{~Hz}, 1 \mathrm{H}$, OCH $H \mathrm{CH}_{2} \mathrm{SiMe}_{3}$ ), 3.69 (ddd, $J=10.1,10.1,6.2 \mathrm{~Hz}, 1 \mathrm{H}, \mathrm{OCH} H \mathrm{CH}_{2} \mathrm{SiMe}_{3}$ ), 3.51 (dd, $J=10.1,7.8 \mathrm{~Hz}, 1$ H, H-2), 3.27 (s, $1 \mathrm{H}, \mathrm{OH}), 1.49$ (s, $3 \mathrm{H}, \mathrm{Me}), 1.14$ - 1.04 (m, $2 \mathrm{H}, \mathrm{OCH}_{2} \mathrm{CH}_{2} \mathrm{SiMe}_{3}$ ), 0.06 (s, $9 \mathrm{H}, \mathrm{SiMe}_{3}$ ); ${ }^{13} \mathrm{C}$ NMR (100 MHz, $\left.\mathrm{CDCl}_{3}\right): \delta$ ppm 167.47, 153.68, 151.22, 150.09, 138.22, 129.73, 129.51, 128.49, 
$127.82,127.73,126.60,126.13,121.49,121.06,103.47,82.23,78.71,76.75,74.88,73.41,68.35,18.61$, 17.11, -1.26; HRMS calcd for $\mathrm{C}_{32} \mathrm{H}_{42} \mathrm{NO}_{9} \mathrm{Si}\left[\mathrm{M}+\mathrm{NH}_{4}\right]^{+}:$612.2629, found 612.2616.

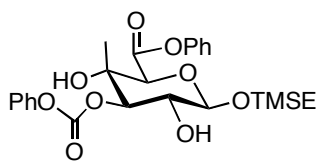

Phenyl [2-(Trimethylsilyl)ethyl 4-C-methyl-3- $O$-phenoxycarbonyl- $\beta$-D-glucopyranosid] uronate (5).

To a stirred solution of $\mathbf{5 . 1 3}$ (460 mg, $0.773 \mathrm{mmol})$ in degassed THF (10 mL) under an atmosphere of argon was added $\mathrm{Pd} / \mathrm{C}(100 \mathrm{mg})$. The suspension was degassed and backfilled with $\mathrm{H}_{2}$ three times then stirred under $\mathrm{H}_{2}(1 \mathrm{~atm})$ overnight. The reaction mixture was then filtered through a pad of celite and concentrated in vacuo. Silica gel chromatography (25\% EtOAc/petroleum ether) afforded the title compound (381 mg, $0.755 \mathrm{mmol}, 98 \%) .{ }^{1} \mathrm{H} \mathrm{NMR}\left(600 \mathrm{MHz}, \mathrm{CDCl}_{3}\right): \delta \mathrm{ppm} 7.41(\mathrm{t}, J=7.9 \mathrm{~Hz}, 2 \mathrm{H}$, $\mathrm{PhH}), 7.37$ (dd, $J=8.5,7.3 \mathrm{~Hz}, 2 \mathrm{H}, \mathrm{PhH}), 7.28$ (t, $J=7.5 \mathrm{~Hz}, 1 \mathrm{H}, \mathrm{PhH}), 7.26$ - $7.21(\mathrm{~m}, 3 \mathrm{H}, \mathrm{PhH}), 7.14$ (d, $J=7.6 \mathrm{~Hz}, 2 \mathrm{H}, \mathrm{PhH}), 5.04$ (d, $J=10.3 \mathrm{~Hz}, 1 \mathrm{H}, \mathrm{H}-3), 4.49$ (d, $J=7.9 \mathrm{~Hz}, 1 \mathrm{H}, \mathrm{H}-1), 4.27$ (s, $1 \mathrm{H}, \mathrm{H}-$ 5), 4.10 (ddd, $J=10.3,10.3,5.9 \mathrm{~Hz}, 1 \mathrm{H}, \mathrm{OCH} H C H_{2} \mathrm{SiMe}_{3}$ ), 3.70 - 3.62 (m, $2 \mathrm{H}, \mathrm{H}-2, \mathrm{OCH}_{\mathrm{CCH}} \mathrm{SiMe}_{3}$ ), 3.29 (s, $1 \mathrm{H}, \mathrm{OH}), 1.48$ (s, $3 \mathrm{H}, \mathrm{Me}), 1.10-0.99$ (m, $\left.2 \mathrm{H}, \mathrm{OCH}_{2} \mathrm{CH}_{2} \mathrm{SiMe}_{3}\right), 0.04\left(\mathrm{~s}, 9 \mathrm{H}, \mathrm{SiMe}_{3}\right) ;{ }^{13} \mathrm{C}$ NMR (125 MHz, $\left.\mathrm{CDCl}_{3}\right)$ : $\delta$ ppm 167.43, 153.91, 151.26, 150.04, 129.76, 129.55, 126.64, 126.19, 121.47, 121.11, 102.91, 82.44, 76.91, 73.40, 71.20, 68.39, 18.30, 17.21, -1.26; HRMS calcd for $\mathrm{C}_{25} \mathrm{H}_{36} \mathrm{NO}_{9} \mathrm{Si}[\mathrm{M}$ $\left.+\mathrm{NH}_{4}\right]^{+}:$522.2159, found 522.2167.

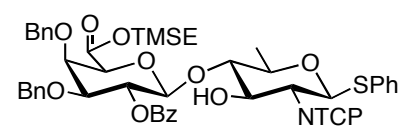

Phenyl [2-(Trimethylsilyl)ethyl 3,4-di- $O$-benzyl-2- $O$-benzoyl- $\beta$-D-galactopyrosyl-uronate]-(1 $\rightarrow 4)$-2deoxy-2-tetrachlorophthalimido-1-thio- $\beta$-D-glucopyranoside (6).

A solution of sulfoxide 2 (187 mg, $0.27 \mathrm{mmol}), 4 \AA$ molecular sieves and 4-allyl-1,2-dimethoxybenzene $(81 \mu \mathrm{L}, 0.54 \mathrm{mmol})$ in methylene chloride $(2.5 \mathrm{ml})$ was stirred at room temperature for $1 \mathrm{~h}$ then cooled to 
$-78{ }^{\circ} \mathrm{C}$ and triflic anhydride $(44 \mu 1,0.27 \mathrm{mmol})$ was slowly added. A solution of acceptor $3(119 \mathrm{mg}, 0.22$ mmol) and 2,6-di-tert-butyl-4-methylpyridine (145 $\mathrm{mg}, 0.70 \mathrm{mmol})$ in methylene chloride $(2 \mathrm{ml})$ was added dropwise via syringe over $10 \mathrm{~min}$. The reaction mixture was warmed to $0{ }^{\circ} \mathrm{C}$ over $1 \mathrm{~h}$ and then quenched with a saturated aqueous $\mathrm{NaHCO}_{3}$ solution. The crude mixture was then diluted with $\mathrm{CH}_{2} \mathrm{Cl}_{2}$ and washed with $\mathrm{H}_{2} \mathrm{O}$ and brine. The organic phase was then dried over $\mathrm{Na}_{2} \mathrm{SO}_{4}$ and concentrated in vacuo. The crude residue was purified by silica gel chromatography (20\% EtOAc/petroleum ether) to afford the title compound (184 mg, $0.20 \mathrm{mmol}, 75 \%)$. ${ }^{1} \mathrm{H}$ NMR (400 MHz, $\left.\mathrm{CDCl}_{3}\right): \delta 8.00-7.04(\mathrm{~m}, 20$ $\mathrm{H}, \mathrm{PhH}), 5.70\left(\mathrm{dd}, J=10.2,8.1 \mathrm{~Hz}, 1 \mathrm{H}, \mathrm{H}-2^{\mathrm{B}}\right), 5.50\left(\mathrm{~d}, J=10.6 \mathrm{~Hz}, 1 \mathrm{H}, \mathrm{H}-1^{\mathrm{C}}\right), 4.98,4.64(\mathrm{ABq}, J=$ 11.7 Hz, $1 \mathrm{H}$ each, $\left.\mathrm{OCH}_{2} \mathrm{Ph}\right), 4.68,4.49\left(\mathrm{ABq}, J=12.4 \mathrm{~Hz}, 1 \mathrm{H}\right.$ each, $\left.\mathrm{OCH}_{2} \mathrm{Ph}\right), 4.59(\mathrm{~d}, J=8.1 \mathrm{~Hz}, 1 \mathrm{H}$, $\left.\mathrm{H}-1^{\mathrm{B}}\right), 4.44\left(\mathrm{dd}, J=10.6,8.4 \mathrm{~Hz}, 1 \mathrm{H}, \mathrm{H}-3^{\mathrm{C}}\right), 4.38\left(\right.$ br s, $\left.1 \mathrm{H}, \mathrm{H}-4^{\mathrm{B}}\right), 4.19(\mathrm{dd}, J=10.6,10.6 \mathrm{~Hz}, 1 \mathrm{H}, \mathrm{H}-$ $\left.\left.2^{\mathrm{C}}\right), 4.14\left(\mathrm{~m}, 1 \mathrm{H}, \mathrm{OCH} H \mathrm{CH}_{2} \mathrm{SiMe}_{3}\right), 4.09\left(\mathrm{~s}, 1 \mathrm{H}, \mathrm{H}-5^{\mathrm{B}}\right), 4.01\left(\mathrm{~m}, 1 \mathrm{H}, \mathrm{OCH} H \mathrm{CH}_{2} \mathrm{SiMe}_{3}\right),\right), 3.72(\mathrm{dd}, J=$ 2.5, $\left.10.2 \mathrm{~Hz}, 1 \mathrm{H}, \mathrm{H}-3^{\mathrm{B}}\right), 3.51\left(\mathrm{~m}, 1 \mathrm{H}, \mathrm{H}^{\mathrm{C}}{ }^{\mathrm{C}}\right), 3.18\left(\mathrm{dd}, J=8.4,8.4 \mathrm{~Hz}, 1 \mathrm{H}, \mathrm{H}-4^{\mathrm{C}}\right), 1.05(\mathrm{~d}, J=5.8 \mathrm{~Hz}, 3$ H, H-6 ${ }^{\mathrm{C}}$ ), $0.72\left(\mathrm{~m}, 2 \mathrm{H}, \mathrm{OCH}_{2} \mathrm{CH}_{2} \mathrm{SiMe}_{3}\right),-0.90$ (s, $\left.9 \mathrm{H}, \mathrm{SiMe}_{3}\right) ;{ }^{13} \mathrm{C} \mathrm{NMR}\left(100 \mathrm{MHz}, \mathrm{CDCl}_{3}\right): \delta 167.2$, $165.2,163.3,162.8,140.3,138.0,137.2,133.6,132.4,130.1,129.9,129.1,128.7,128.2,127.9,127.6$, $102.2,88.9,83.0,78.9,74.9,74.6,74.1,73.8,72.1,71.3,70.7,64.8,56.3,17.8,17.4,-1.5$; LRMS calcd for $\mathrm{C}_{52} \mathrm{H}_{51} \mathrm{Cl}_{4} \mathrm{NO}_{12} \mathrm{SSiNa}[\mathrm{M}+\mathrm{Na}]^{+}$: 1104.2, found 1104.2.

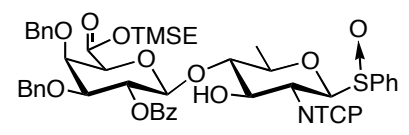

\section{Phenyl [2-(Trimethylsilyl)ethyl 3,4-di- $O$-benzyl-2- $O$-benzoyl- $\beta$-D-galactopyrosyluronate]-(1 $\rightarrow 4)-2$ -} deoxy-2-tetrachlorophthalimido-1-thio- $\beta$-D-glucopyranoside $S$-Oxide (7).

To a solution of sulfide 6 (500 mg, $0.46 \mathrm{mmol})$ in $\mathrm{CH}_{2} \mathrm{Cl}_{2}$ was added $m \mathrm{CPBA} \quad(100 \mathrm{mg}, \sim 75 \%, 0.43$ $\mathrm{mmol}$ ) at $-78^{\circ} \mathrm{C}$. The reaction mixture was warmed to $0{ }^{\circ} \mathrm{C}$ over $2 \mathrm{~h}$ and quenched with saturated aqueous $\mathrm{NaHCO}_{3}$. The mixture was then extracted with $\mathrm{CH}_{2} \mathrm{Cl}_{2}$. The organic layer was washed with brine and dried over $\mathrm{Na}_{2} \mathrm{SO}_{4}$. After concentration of the organic phase in vacuo, he product was purified by silica gel chromatography (30\% EtOAc/petroleum ether) to afford the title compound as a mixture of 
diastereomeric sulfoxides (350 mg, $0.32 \mathrm{mmol}, 70 \%)$. Major: ${ }^{1} \mathrm{H} \mathrm{NMR}\left(400 \mathrm{MHz}, \mathrm{CDCl}_{3}\right): \delta 8.00-$ $7.04(\mathrm{~m}, 20 \mathrm{H}, \mathrm{PhH}), 5.68\left(\mathrm{dd}, J=9.2,8.0 \mathrm{~Hz}, 1 \mathrm{H}, \mathrm{H}-2^{\mathrm{B}}\right), 5.06\left(\mathrm{~d}, J=10.2 \mathrm{~Hz}, 1 \mathrm{H}, \mathrm{H}-1^{\mathrm{C}}\right), 4.96,4.62$ $\left(\mathrm{ABq}, J=11.7 \mathrm{~Hz}, 1 \mathrm{H}\right.$ each, $\left.\mathrm{CH}_{2} \mathrm{Ph}\right), 4.72\left(\mathrm{dd}, J=10.2,10.2 \mathrm{~Hz}, 1 \mathrm{H}, \mathrm{H}-2^{\mathrm{C}}\right), 4.68,4.48(\mathrm{ABq}, J=12.4$ $\mathrm{Hz}, 1 \mathrm{H}$ each, $\left.\mathrm{CH}_{2} \mathrm{Ph}\right), 4.59\left(\mathrm{~d}, J=8.0 \mathrm{~Hz}, 1 \mathrm{H}, \mathrm{H}-1^{\mathrm{B}}\right), 4.40\left(\mathrm{dd}, J=10.2,8.0 \mathrm{~Hz}, 1 \mathrm{H}, \mathrm{H}-3^{\mathrm{C}}\right), 4.38($ br s, 1 $\mathrm{H}, \mathrm{H}-4^{\mathrm{B}}$ ), $4.34\left(\mathrm{~m}, 1 \mathrm{H}, \mathrm{OCH} H \mathrm{CH}_{2} \mathrm{SiMe}_{3}\right), 4.12\left(\mathrm{~s}, 1 \mathrm{H}, \mathrm{H}-5^{\mathrm{B}}\right), 4.02\left(\mathrm{~m}, 1 \mathrm{H}, \mathrm{OCH} H \mathrm{CH}_{2} \mathrm{SiMe}_{3}\right)$,), 4.75 $\left(\mathrm{dd}, J=2.5,9.2 \mathrm{~Hz}, 1 \mathrm{H}, \mathrm{H}-3^{\mathrm{B}}\right), 3.41\left(\mathrm{dd}, J=6.2,8.0 \mathrm{~Hz}, 1 \mathrm{H}, \mathrm{H}-5^{\mathrm{C}}\right), 3.17(\mathrm{dd}, J=8.0,8.0 \mathrm{~Hz}, 1 \mathrm{H}, \mathrm{H}-$ $\left.4^{\mathrm{C}}\right), 1.00\left(\mathrm{~d}, J=6.2 \mathrm{~Hz}, 3 \mathrm{H}, \mathrm{H}-6^{\mathrm{C}}\right), 0.80\left(\mathrm{~m}, 2 \mathrm{H}, \mathrm{OCH}_{2} \mathrm{CH}_{2} \mathrm{SiMe}_{3}\right),-0.90\left(\mathrm{~s}, 9 \mathrm{H}, \mathrm{SiMe}_{3}\right) ;{ }^{13} \mathrm{C} \mathrm{NMR}$ $\left(100 \mathrm{MHz}, \mathrm{CDCl}_{3}\right): \delta 167.2,165.2,163.3,162.3,140.3,139.9,138.0,137.2,133.6,130.6,130.0,129.8$, $128.8,128.6,128.2,128.0,127.9,127.3,124.6,102.2,89.2,88.1,79.0,75.3,74.9,74.1,73.8,72.1,71.3$, 70.6, 64.8, 50.7, 17.4, -1.5. Minor: ${ }^{1} \mathrm{H}$ NMR $\left(400 \mathrm{MHz}, \mathrm{CDCl}_{3}\right): \delta 8.00-7.04(\mathrm{~m}, 20 \mathrm{H}, \mathrm{PhH}), 5.66(\mathrm{dd}$, $\left.J=7.7,9,9 \mathrm{~Hz}, 1 \mathrm{H}, \mathrm{H}-2^{\mathrm{B}}\right), 5.08\left(\mathrm{~d}, J=10.6 \mathrm{~Hz}, 1 \mathrm{H}, \mathrm{H}-1^{\mathrm{C}}\right), 4.95,4.62(\mathrm{ABq}, J=11.7 \mathrm{~Hz}, 1 \mathrm{H}$ each, $\left.\mathrm{CH}_{2} \mathrm{Ph}\right), 4.66,4.45\left(\mathrm{ABq}, J=12.5 \mathrm{~Hz}, 1 \mathrm{H}\right.$ each, $\left.\mathrm{CH}_{2} \mathrm{Ph}\right), 4.53\left(\mathrm{~d}, J=7.7 \mathrm{~Hz}, 1 \mathrm{H}, \mathrm{H}-1^{\mathrm{B}}\right), 4.50(\mathrm{dd}, J=$ 10.7, $\left.10.7 \mathrm{~Hz}, 1 \mathrm{H}, \mathrm{H}-2^{\mathrm{C}}\right), 4.35\left(\mathrm{dd}, J=10.7,8.1 \mathrm{~Hz}, 1 \mathrm{H}, \mathrm{H}-3^{\mathrm{C}}\right), 4.34\left(\right.$ br s, $\left.1 \mathrm{H}, \mathrm{H}-4^{\mathrm{B}}\right), 4.38-4.18(\mathrm{~m}, 1$ $\left.\mathrm{H}, \mathrm{OCH} H \mathrm{CH}_{2} \mathrm{SiMe}_{3}\right), 4.06\left(\mathrm{~s}, 1 \mathrm{H}, \mathrm{H}-5^{\mathrm{B}}\right), 4.03-3.90$ (m, $\left.1 \mathrm{H}, \mathrm{OCH} H C H_{2} \mathrm{SiMe}_{3}\right), 3.70$ (dd, $J=9.9,2.2$ $\left.\mathrm{Hz}, 1 \mathrm{H}, \mathrm{H}-3^{\mathrm{B}}\right), 3.40\left(\mathrm{~m}, 1 \mathrm{H}, \mathrm{H}-5^{\mathrm{C}}\right), 3.11\left(\mathrm{dd}, J=8.1,8.1 \mathrm{~Hz}, 1 \mathrm{H}, \mathrm{H}-4^{\mathrm{C}}\right), 0.90\left(\mathrm{~d}, J=6.2 \mathrm{~Hz}, 3 \mathrm{H}, \mathrm{H}-6^{\mathrm{C}}\right)$, $0.82-0.75\left(\mathrm{~m}, 2 \mathrm{H}, \mathrm{OCH}_{2} \mathrm{CH}_{2} \mathrm{SiMe}_{3}\right),-0.90\left(\mathrm{~s}, 9 \mathrm{H}, \mathrm{SiMe}_{3}\right) ;{ }^{13} \mathrm{C} \mathrm{NMR}\left(100 \mathrm{MHz}, \mathrm{CDCl}_{3}\right): \delta 167.2$, $165.1,163.9,162.3,140.4,139.2,138.0,137.2,133.6,131.5,130.3,130.0,129.9,129.6,128.9,128.5$, $128.3,127.6,127.4,125.9,124.6,102.2,88.4,87.4,78.8,75.6,75.0,74.2,73.7,72.1,71.3,70.9,64.8$, $52.1,94.7,88.8,82.3,78.9,75.8,75.2,74.8,74.0,73.6,73.5,73.2,72.1,71.2,70.3,69.0,68.4,64.8,57.6$, 56.3, 55.5, 55.3, 42.7, 20.9, 18.4, 17.4, 17.3, 16.7, -1.35, -1.54; LRMS calcd for $\mathrm{C}_{52} \mathrm{H}_{51} \mathrm{Cl}_{4} \mathrm{NO}_{13} \mathrm{SSiNa}[\mathrm{M}$ $+\mathrm{Na}]^{+}: 1120.2$, found 1120.2

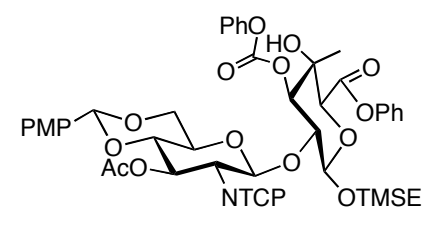




\section{Phenyl \{3-O-Acetyl-2-deoxy-4,6- $O$-p-methoxybenzylidene-2-tetrachlorophthalimido- $\beta$-D-}

glucopyranosyl-(1 $\rightarrow 2)$-[2-(trimethylsilyl)ethyl 4-C-methyl-3- $O$-phenoxycarbonyl- $\beta$-Dglucopyranosid]\} uronate (8).

To a flame dried flask was added acceptor $5(216 \mathrm{mg}, 0.428 \mathrm{mmol})$ and 4-allyl-1,2-dimethoxybenzene (736 $\mu \mathrm{L}, 0.428 \mathrm{mmol})$. In a second flame dried flask was added sulfoxide 4 (612 $\mathrm{mg}, 0.855 \mathrm{mmol})$ and 2,6-di-tert-butyl-4-methylpyridine (264 mg, $1.29 \mathrm{mmol})$. Both flasks were placed under vacuum for $2 \mathrm{~h}$ then switched to an atmosphere of argon. To the acceptor flask was added $\mathrm{CH}_{2} \mathrm{Cl}_{2}(8 \mathrm{~mL})$ and $4 \AA$ molecular sieves then the suspension was stirred at room temperature for $1 \mathrm{~h}$. The suspension was cooled to $-42{ }^{\circ} \mathrm{C}$ and treated with triflic anhydride $(180 \mu \mathrm{L}, 0.302 \mathrm{mmol})$. The sulfoxide in $\mathrm{CH}_{2} \mathrm{Cl}_{2}(4 \mathrm{~mL})$ was then added to the acceptor solution by syringe pump over $1 \mathrm{~h}$ at $-42{ }^{\circ} \mathrm{C}$. After addition of sulfoxide, the reaction mixture was stirred for $1 \mathrm{~h}$ at $-42{ }^{\circ} \mathrm{C}$ then quenched with triethylamine $(200 \mu \mathrm{L})$ and poured into a separatory funnel containing $\mathrm{H}_{2} \mathrm{O}$ and $\mathrm{CH}_{2} \mathrm{Cl}_{2}$. The organic phase was collected, extracted from brine, dried over $\mathrm{Na}_{2} \mathrm{SO}_{4}$, and concentrated in vacuo. The crude residue was purified by silica gel chromatography (4\% EtOAc/toluene) to afford the title compound (392 mg, $0.358 \mathrm{mmol}, 84 \%) .{ }^{1} \mathrm{H}$ NMR $\left(600 \mathrm{MHz}, \mathrm{CDCl}_{3}\right): \delta$ ppm $7.42-7.35(\mathrm{~m}, 6 \mathrm{H}, \mathrm{ArH}), 7.29-7.22(\mathrm{~m}, 2 \mathrm{H}, \mathrm{ArH}), 7.17(\mathrm{~d}, J=8.8 \mathrm{~Hz}, 2 \mathrm{H}$, ArH), 7.10 (d, $J=8.8 \mathrm{~Hz}, 2 \mathrm{H}, \operatorname{ArH}), 6.89$ (d, $J=8.5 \mathrm{~Hz}, 2 \mathrm{H}, \mathrm{ArH}), 5.79\left(\mathrm{~d}, J=8.5 \mathrm{~Hz}, 1 \mathrm{H}, \mathrm{H}-1^{\mathrm{E}}\right), 5.71$ $\left(\mathrm{dd}, J=9.7,9.7 \mathrm{~Hz}, 1 \mathrm{H}, \mathrm{H}-3^{\mathrm{E}}\right), 5.51(\mathrm{~s}, 1 \mathrm{H}, \mathrm{CHPMP}), 4.91\left(\mathrm{~d}, J=10.0 \mathrm{~Hz}, 1 \mathrm{H}, \mathrm{H}-3^{\mathrm{F}}\right), 4.53(\mathrm{~d}, J=7.6$ $\left.\mathrm{Hz}, 1 \mathrm{H}, \mathrm{H}-1^{\mathrm{F}}\right), 4.38\left(\mathrm{dd}, J=10.5,5.0 \mathrm{~Hz}, 1 \mathrm{H}, \mathrm{H}-6^{\mathrm{E}}\right), 4.30\left(\mathrm{dd}, J=10.1,8.3 \mathrm{~Hz}, 1 \mathrm{H}, \mathrm{H}-2^{\mathrm{E}}\right), 4.13(\mathrm{~s}, 1 \mathrm{H}$, $\left.\mathrm{H}-5^{\mathrm{F}}\right), 4.07\left(\mathrm{ddd}, J=9.9,9.9,6.3 \mathrm{~Hz}, 1 \mathrm{H}, \mathrm{OCH} H \mathrm{CH}_{2} \mathrm{SiMe}_{3}\right), 3.85\left(\mathrm{dd}, J=10.3,10.3 \mathrm{~Hz}, 1 \mathrm{H}, \mathrm{H}-4^{\mathrm{E}}\right)$, $3.80(\mathrm{~s}, 3 \mathrm{H}, \mathrm{OMe}), 3.82-3.76\left(\mathrm{~m}, 1 \mathrm{H}, \mathrm{H}-6^{\mathrm{E}}\right), 3.76-3.67\left(\mathrm{~m}, 3 \mathrm{H}, \mathrm{OCH} H \mathrm{CH}_{2} \mathrm{SiMe}_{3}, \mathrm{H}-2^{\mathrm{F}}, \mathrm{E}^{5}\right), 3.12(\mathrm{~s}, 1$ $\mathrm{H}, \mathrm{OH}), 1.90(\mathrm{~s}, 3 \mathrm{H}, \mathrm{OAc}), 1.32(\mathrm{~s}, 3 \mathrm{H}, \mathrm{Me}), 1.12$ - $1.00\left(\mathrm{~m}, 2 \mathrm{H}, \mathrm{OCH}_{2} \mathrm{CH}_{2} \mathrm{SiMe}_{3}\right), 0.09$ (s, $9 \mathrm{H}$, $\left.\mathrm{SiMe}_{3}\right) ;{ }^{13} \mathrm{C}$ NMR $\left(100 \mathrm{MHz}, \mathrm{CDCl}_{3}\right): \delta \mathrm{ppm} 170.89,167.24,164.02,163.18,160.39,153.07,151.07$, $150.00,140.52,140.21,130.14,129.74,129.39,129.34,127.75,127.38,127.10,126.64,126.25,121.36$, $121.15,121.05,113.78,101.96,101.92,98.42,81.91,78.96,77.02,76.27,73.74,70.39,68.79,68.18$, 
$66.58,56.74,55.42,20.72,18.35,16.88,-1.11$; HRMS calcd for $\mathrm{C}_{49} \mathrm{H}_{49} \mathrm{Cl}_{4} \mathrm{NO}_{17} \mathrm{SiNa}[\mathrm{M}+\mathrm{Na}]^{+}$: 1114.1422, found 1114.1392 .

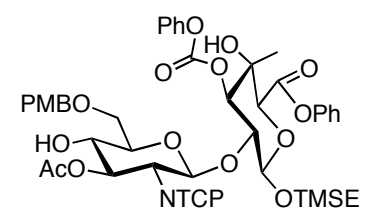

Phenyl \{3-O-Acetyl-2-deoxy-6- $O$-p-methoxybenzyl-2-tetrachlorophthalimido- $\beta$-D-glucopyranosyl$(1 \rightarrow 2)$-[2-(trimethylsilyl)ethyl 4-C-methyl-3- $O$-phenoxycarbonyl- $\beta$-D-glucopyranosid]\} uronate (9).

Disaccharide 8 (522 mg, $0.477 \mathrm{mmol})$ was dissolved in THF $(10 \mathrm{~mL})$ then cooled to $-65{ }^{\circ} \mathrm{C}$. A solution of $1 \mathrm{M} \mathrm{BH}_{3} \cdot \mathrm{THF}(2.38 \mathrm{~mL}, 2.38 \mathrm{mmol})$ was then added and the mixture was stirred for $5 \mathrm{~min}$ then treated with dibutylboron triflate $\left(1.19 \mathrm{~mL}\right.$ of a $1 \mathrm{M}$ complex in $\left.\mathrm{CH}_{2} \mathrm{Cl}_{2}, 1.19 \mathrm{mmol}\right){ }^{5}$ The reaction mixture was kept at $-65{ }^{\circ} \mathrm{C}$ for $6 \mathrm{~h}$ then quenched with triethylamine $(1 \mathrm{~mL})$ and slowly poured into a separatory funnel containing saturated aqueous $\mathrm{NaHCO}_{3}$ and $\mathrm{CH}_{2} \mathrm{Cl}_{2}$. The separatory funnel was carefully shaken and vented $\left(\mathrm{H}_{2}\right.$ gas is produced as the borane is quenched) then the organic phase was collected. The organic phase was washed with $\mathrm{H}_{2} \mathrm{O}$ and brine, dried over $\mathrm{Na}_{2} \mathrm{SO}_{4}$, and concentrated in vacuo. The crude residue was purified by silica gel chromatography (30\% EtOAc/petroleum ether) to afford the title compound (432 mg, $0.394 \mathrm{mmol}, 83 \%) .{ }^{1} \mathrm{H} \mathrm{NMR}\left(600 \mathrm{MHz}, \mathrm{CDCl}_{3}\right): \delta \mathrm{ppm} 7.39(\mathrm{dd}, J=8.5,7.6 \mathrm{~Hz}, 2$ H, ArH), 7.35 (dd, $J=8.5,7.6 \mathrm{~Hz}, 2 \mathrm{H}, \mathrm{ArH}), 7.29-7.25$ (m, $3 \mathrm{H}, \mathrm{ArH}), 7.23$ (t, J= 7.3 Hz, $1 \mathrm{H}, \mathrm{ArH})$, $7.12-7.08(\mathrm{~m}, 4 \mathrm{H}, \mathrm{ArH}), 6.90(\mathrm{~d}, J=8.8 \mathrm{~Hz}, 2 \mathrm{H}, \mathrm{ArH}), 5.72\left(\mathrm{~d}, J=8.5 \mathrm{~Hz}, 1 \mathrm{H}, \mathrm{H}-1^{\mathrm{E}}\right), 5.45(\mathrm{dd}, J=$ $\left.10.8,8.8 \mathrm{~Hz}, 1 \mathrm{H}, \mathrm{H}-3^{\mathrm{E}}\right), 4.88\left(\mathrm{~d}, J=10.0 \mathrm{~Hz}, 1 \mathrm{H}, \mathrm{H}-3^{\mathrm{F}}\right), 4.59,4.52(\mathrm{ABq}, J=11.4 \mathrm{~Hz}, 1 \mathrm{H}$ each, $\left.\mathrm{OCH}_{2} \mathrm{PMP}\right), 4.55\left(\mathrm{~d}, J=7.6 \mathrm{~Hz}, 1 \mathrm{H}, \mathrm{H}-1^{\mathrm{F}}\right), 4.22\left(\mathrm{dd}, J=10.8,8.5 \mathrm{~Hz}, 1 \mathrm{H}, \mathrm{H}-2^{\mathrm{E}}\right), 4.12\left(\mathrm{~s}, 1 \mathrm{H}, \mathrm{H}-5^{\mathrm{F}}\right)$, $4.03\left(\mathrm{ddd}, J=11.2,9.2,6 \mathrm{~Hz}, 1 \mathrm{H}, \mathrm{OCH} H \mathrm{CH}_{2} \mathrm{SiMe}_{3}\right), 3.91\left(\mathrm{ddd}, J=8.5,8.5,1.5 \mathrm{~Hz}, 1 \mathrm{H}, \mathrm{H}-4^{\mathrm{E}}\right), 3.87$ $\left(\mathrm{dd}, J=9.4,3.5 \mathrm{~Hz}, 1 \mathrm{H}, \mathrm{H}-6^{\mathrm{E}}\right), 3.81(\mathrm{~s}, 3 \mathrm{H}, \mathrm{OMe}), 3.76-3.66\left(\mathrm{~m}, 4 \mathrm{H}, \mathrm{OCH} H \mathrm{CH}_{2} \mathrm{SiMe}_{3}, \mathrm{H}-2^{\mathrm{F}}, 5^{\mathrm{E}}, 6^{\mathrm{E}}\right)$, $3.08(\mathrm{~s}, 1 \mathrm{H}, \mathrm{OH}), 2.99$ (d, $J=2.6 \mathrm{~Hz}, 1 \mathrm{H}, \mathrm{OH}), 1.93$ (s, $3 \mathrm{H}, \mathrm{OAc}), 1.33$ (s, $3 \mathrm{H}, \mathrm{Me}), 1.07$ - 1.01 (m, 1 $\left.\mathrm{H}, \mathrm{OCH}_{2} \mathrm{CH} H \mathrm{SiMe}_{3}\right), 0.99-0.94$ (m, $\left.1 \mathrm{H}, \mathrm{OCH}_{2} \mathrm{CH} H \mathrm{SiMe}_{3}\right), 0.04$ (s, $\left.9 \mathrm{H}, \mathrm{SiMe}_{3}\right) ;{ }^{13} \mathrm{C} \mathrm{NMR}(100 \mathrm{MHz}$, $\left.\mathrm{CDCl}_{3}\right): \delta$ ppm 171.32, 167.20, 163.94, 163.21, 159.57, 153.13, 150.95, 150.00, 140.35, 140.12, 129.72, 
$129.69,129.47,129.39,127.38,127.20,126.60,126.24,121.37,121.17,114.07,102.05,97.99,81.81$, $76.77,76.30,73.97,73.89,73.75,73.71,71.40,69.97,68.05,55.93,55.39,20.77,18.39,16.80,-1.19$; HRMS calcd for $\mathrm{C}_{49} \mathrm{H}_{55} \mathrm{Cl}_{4} \mathrm{~N}_{2} \mathrm{O}_{17} \mathrm{Si}\left[\mathrm{M}+\mathrm{NH}_{4}\right]^{+}:$1111.2024, found 1111.1980.

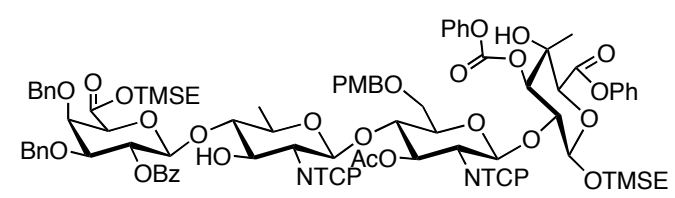

Phenyl \{[2-(Trimethylsilyl)ethyl 2- $O$-benzoyl-3,4-di- $O$-benzyl- $\beta$-D-galactopyranosyluronate]-(1 $\rightarrow 4)$ 2,6-dideoxy-2-tetrachlorophthalimido- $\beta$-D-glucopyranosyl-(1 $\rightarrow 4)-3-O$-acetyl-2-deoxy-6-O-pmethoxybenzyl-2-tetrachlorophthalimido- $\beta$-D-glucopyranosyl-(1 $\rightarrow 2)$-[2-(Trimethylsilyl)ethyl 4-Cmethyl-3- $O$-phenoxycarbonyl- $\beta$-D-glucopyranosid] $\}$ uronate (10).

To a flame dried flask was added acceptor 9 (95 mg, $0.087 \mathrm{mmol})$, 4-allyl-1,2-dimethoxybenzene (149 $\mu \mathrm{L}, 0.87 \mathrm{mmol}$ ), and 2,6-di-tert-butyl-4-methylpyridine $(53 \mathrm{mg}, 0.26 \mathrm{mmol})$. The flask was placed under vacuum for $2 \mathrm{~h}$ then switched to an argon atmosphere. After addition of $\mathrm{CH}_{2} \mathrm{Cl}_{2}(2 \mathrm{~mL})$ and $4 \AA$ molecular sieves, the suspension was stirred at room temperature for $1 \mathrm{~h}$ then chilled to $-65{ }^{\circ} \mathrm{C}$ and treated with triflic anhydride $(74 \mu \mathrm{L}, 0.46 \mathrm{mmol})$. A solution of sulfoxide 9 (97 $\mathrm{mg}, 0.088 \mathrm{mmol})$ in $\mathrm{CH}_{2} \mathrm{Cl}_{2}(1 \mathrm{~mL})$ was added dropwise by syringe pump over $1 \mathrm{~h}$. The reaction mixture was then allowed to slowly warm to $-30{ }^{\circ} \mathrm{C}$ over an additional $1 \mathrm{~h}$, then was quenched with triethylamine $(50 \mu \mathrm{L})$ and poured into a separatory funnel containing $\mathrm{H}_{2} \mathrm{O}$ and $\mathrm{CH}_{2} \mathrm{Cl}_{2}$. The organic phase was collected then extracted from brine, dried over $\mathrm{Na}_{2} \mathrm{SO}_{4}$, and concentrated in vacuo. The crude residue was purified by silica gel chromatography (gradient 2-4\% EtOAc/ $\mathrm{CH}_{2} \mathrm{Cl}_{2}$ ) to afford the title compound (90 $\mathrm{mg}, 0.043 \mathrm{mmol}, 50 \%$ ) along with recovered acceptor $(24 \mathrm{mg}, 0.022,25 \%) .{ }^{1} \mathrm{H}$ NMR $\left(600 \mathrm{MHz}, \mathrm{CDCl}_{3}\right): \delta \mathrm{ppm} 7.95(\mathrm{dd}, J=$ 7.3, 1.2 Hz, 2 H, BzH), 7.57 (t, $J=7.5 \mathrm{~Hz}, 1 \mathrm{H}, \mathrm{BzH}), 7.41$ (t, $J=7.9 \mathrm{~Hz}, 2 \mathrm{H}, \mathrm{BzH}), 7.36$ (t, $J=7.9 \mathrm{~Hz}$, $2 \mathrm{H}, \mathrm{PhH}), 7.34$ (t, $J=7.9 \mathrm{~Hz}, 2 \mathrm{H}, \mathrm{PhH}), 7.28-7.16(\mathrm{~m}, 8 \mathrm{H}, \mathrm{PhH}), 7.13-7.12(\mathrm{~m}, 6 \mathrm{H}, \mathrm{PhH}), 7.06(\mathrm{~d}, J$ $=8.8 \mathrm{~Hz}, 2 \mathrm{H}, \mathrm{PhH}), 6.85(\mathrm{~d}, J=8.5 \mathrm{~Hz}, 2 \mathrm{H}, \mathrm{PMPH}), 6.76$ (d, $J=8.5 \mathrm{~Hz}, 2 \mathrm{H}, \mathrm{PMPH}), 5.66(\mathrm{dd}, J=8.8$, 
$\left.8.8 \mathrm{~Hz}, 1 \mathrm{H}, \mathrm{H}-2^{\mathrm{B}}\right), 5.57\left(\mathrm{dd}, J=10.3,8.5 \mathrm{~Hz}, 1 \mathrm{H}, \mathrm{H}-3^{\mathrm{E}}\right), 5.55\left(\mathrm{~d}, J=8.5 \mathrm{~Hz}, 1 \mathrm{H}, \mathrm{H}-1^{\mathrm{E}}\right), 5.30(\mathrm{~d}, J=8.5$ $\left.\mathrm{Hz}, 1 \mathrm{H}, \mathrm{H}-1^{\mathrm{C}}\right), 4.97,4.63\left(\mathrm{ABq}, J=11.7 \mathrm{~Hz}, 1 \mathrm{H}\right.$ each, $\left.\mathrm{OCH}_{2} \mathrm{Ph}\right), 4.81\left(\mathrm{~d}, J=10.0 \mathrm{~Hz}, 1 \mathrm{H}, \mathrm{H}-3^{\mathrm{F}}\right), 4.67$ (br s, $\left.1 \mathrm{H}, \mathrm{OH}-3^{\mathrm{C}}\right), 4.66,4.47\left(\mathrm{ABq}, J=12.3 \mathrm{~Hz}, 1 \mathrm{H}\right.$ each, $\left.\mathrm{OCH}_{2} \mathrm{Ph}\right), 4.54\left(\mathrm{~d}, J=7.6 \mathrm{~Hz}, 1 \mathrm{H}, \mathrm{H}-1^{\mathrm{B}}\right)$, $4.42\left(\mathrm{~d}, J=7.6 \mathrm{~Hz}, 1 \mathrm{H}, \mathrm{H}-1^{\mathrm{F}}\right), 4.39-4.35\left(\mathrm{~m}, 1 \mathrm{H}, \mathrm{H}^{\mathrm{C}}{ }^{\mathrm{C}}\right), 4.37,4.20(\mathrm{ABq}, J=11.5 \mathrm{~Hz}, 1 \mathrm{H}$ each, $\left.\mathrm{OCH}_{2} \mathrm{PMP}\right), 4.34\left(\right.$ br s, $\left.1 \mathrm{H}, \mathrm{H}-4^{\mathrm{B}}\right), 4.09\left(\mathrm{dd}, J=10.5,8.5 \mathrm{~Hz}, 1 \mathrm{H}, \mathrm{H}-2^{\mathrm{E}}\right), 4.15-4.07(\mathrm{~m}, 1 \mathrm{H}$, $\left.\mathrm{OCHHCH}_{2} \mathrm{SiMe}_{3}\right), 4.06\left(\mathrm{~s}, 1 \mathrm{H}, \mathrm{H}-5^{\mathrm{F}}\right), 4.05\left(\mathrm{~s}, 1 \mathrm{H}, \mathrm{H}-5^{\mathrm{B}}\right), 4.06-3.96\left(\mathrm{~m}, 3 \mathrm{H}, \mathrm{H}-2^{\mathrm{C}}, 4^{\mathrm{E}}\right.$, $\mathrm{OCH} H C H_{2} \mathrm{SiMe}_{3}$ ), 3.89 - $3.83\left(\mathrm{~m}, 1 \mathrm{H}, \mathrm{OCH} H \mathrm{CH}_{2} \mathrm{SiMe}_{3}\right), 3.82$ (s, $\left.3 \mathrm{H}, \mathrm{OMe}\right), 3.73$ (dd, $J=12.0,2.3 \mathrm{~Hz}$, $\left.1 \mathrm{H}, \mathrm{H}-6^{\mathrm{E}}\right), 3.70-3.68\left(\mathrm{~m}, 1 \mathrm{H}, \mathrm{H}-2^{\mathrm{E}}\right), 3.66\left(\mathrm{dd}, J=10.0,7.6 \mathrm{~Hz}, 1 \mathrm{H}, \mathrm{H}-2^{\mathrm{F}}\right), 3.54-3.46(\mathrm{~m}, 3 \mathrm{H}, \mathrm{H}-$ $\left.5^{\mathrm{E}}, \mathrm{H}-6^{\mathrm{E}}, \mathrm{OCHHCH}_{2} \mathrm{SiMe}_{3}\right), 3.33\left(\mathrm{dd}, J=9.1,5.9 \mathrm{~Hz}, 1 \mathrm{H}, \mathrm{H}-5^{\mathrm{C}}\right), 3.06\left(\mathrm{~s}, 1 \mathrm{H}, \mathrm{OH}-4^{\mathrm{F}}\right), 3.05(\mathrm{dd}, J=9.1$, $\left.9.1 \mathrm{~Hz}, 1 \mathrm{H}, \mathrm{H}-4^{\mathrm{C}}\right), 1.81(\mathrm{~s}, 3 \mathrm{H}, \mathrm{OAc}), 1.29(\mathrm{~s}, 3 \mathrm{H}, \mathrm{Me}), 0.86\left(\mathrm{~d}, J=5.9 \mathrm{~Hz}, 3 \mathrm{H}, \mathrm{H}-6^{\mathrm{C}}\right), 0.70-0.69$ (m, $4 \mathrm{H}, 2 \times \mathrm{OCH}_{2} \mathrm{CH}_{2} \mathrm{SiMe}_{3}$ ), -0.103, -0.105 (s, $9 \mathrm{H}$ each, $\left.\mathrm{SiMe}_{3}\right) ;{ }^{13} \mathrm{C} \mathrm{NMR}\left(125 \mathrm{MHz}, \mathrm{CDCl}_{3}\right): \delta \mathrm{ppm}$ $170.46,167.28,167.10,165.16,163.98,163.70,163.37,163.29,158.94,153.01,151.03,150.02,140.38$, $139.97,139.73,139.68,137.91,137.04,133.56,130.12,129.96,129.84,129.72,129.58,129.53,129.37$, $128.59,128.35,128.17,128.09,127.97,127.86,127.69,127.49,127.35,127.16,126.62,126.22,121.37$, $121.27,113.68,102.25,101.89,97.85,97.82,88.85,82.25,78.90,76.26,76.11,75.17,75.07,74.79$, $74.01,73.74,73.58,73.53,72.02,71.20,70.22,68.91,67.99,67.58,64.74,57.54,56.32,55.23,20.85$, 18.25, 17.29, 17.25, 16.91, -1.36, -1.63; LRMS calcd for $\mathrm{C}_{95} \mathrm{H}_{96} \mathrm{Cl}_{8} \mathrm{~N}_{2} \mathrm{O}_{29} \mathrm{Si}_{2} \mathrm{Na}[\mathrm{M}+\mathrm{Na}]^{+}: 2087.3$, found 2087.4.

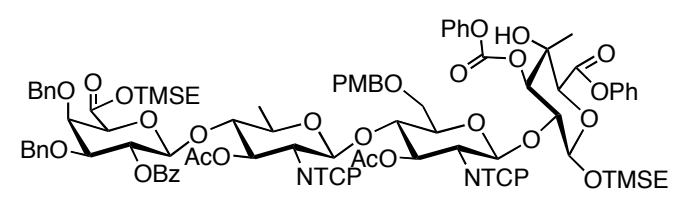

Phenyl \{[2-(Trimethylsilyl)ethyl 2- $O$-benzoyl-3,4-di- $O$-benzyl- $\beta$-D-galactopyranosyluronate]-(1 $\rightarrow 4)$ 3- $O$-acetyl-2,6-dideoxy-2-tetrachlorophthalimido- $\beta$-D-glucopyranosyl-(1 $\rightarrow 4)-3-O$-acetyl-2-deoxy-6O-p-methoxybenzyl-2-tetrachlorophthalimido- $\beta$-D-glucopyranosyl-(1 $\rightarrow 2)$-[2-(trimethylsilyl)ethyl 4C-methyl-3- $O$-phenoxycarbonyl- $\beta$-D-glucopyranosid] $\}$ uronate. 
To a solution of $\mathbf{1 0}(118 \mathrm{mg}, 0.057 \mathrm{mmol})$ in pyridine $(5 \mathrm{~mL})$ was added acetic anhydride $(81 \mu \mathrm{L}, 0.857$ $\mathrm{mmol}$ ) and the reaction was stirred at room temperature overnight. After concentration in vacuo, the crude residue was purified by silica gel chromatography $(30 \%$ EtOAc/petroleum ether) to afford the title compound (112 mg, $0.053 \mathrm{mmol}, 93 \%) .{ }^{1} \mathrm{H} \mathrm{NMR}\left(600 \mathrm{MHz}, \mathrm{CDCl}_{3}\right): \delta \mathrm{ppm} 7.95(\mathrm{~d}, J=7.3 \mathrm{~Hz}, 2 \mathrm{H}$, BzH), 7.56 (t, $J=7.3 \mathrm{~Hz}, 1 \mathrm{H}, \mathrm{BzH}), 7.42$ (t, $J=7.8 \mathrm{~Hz}, 2 \mathrm{H}, \mathrm{BzH}), 7.37$ (t, $J=7.8 \mathrm{~Hz}, 2 \mathrm{H}, \mathrm{PhH}), 7.35$ (t, J=7.8 Hz, 2 H, PhH), 7.29 - 7.17 (m, 8 H, PhH), 7.14 (d, J=4.4 Hz, 6 H, PhH), 7.06 (d, J= 8.5 Hz, 2 H, PhH), 6.85 (d, J=8.2 Hz, 2 H, PMPH), 6.76 (d, $J=8.5$ Hz, 2 H, PMPH), 5.60 - 5.54 (m, 3 H, H$\left.2^{\mathrm{B}}, 3^{\mathrm{C}}, 3^{\mathrm{E}}\right), 5.55\left(\mathrm{~d}, J=8.5 \mathrm{~Hz}, 1 \mathrm{H}, \mathrm{H}-1^{\mathrm{E}}\right), 5.42\left(\mathrm{~d}, J=8.2 \mathrm{~Hz}, 1 \mathrm{H}, \mathrm{H}-1^{\mathrm{C}}\right), 4.94,4.59(\mathrm{ABq}, J=11.7 \mathrm{~Hz}, 1$ $\mathrm{H}$ each, $\left.\mathrm{OCH}_{2} \mathrm{Ph}\right), 4.81\left(\mathrm{~d}, J=10.0 \mathrm{~Hz}, 1 \mathrm{H}, \mathrm{H}-3^{\mathrm{F}}\right), 4.65,4.46\left(\mathrm{ABq}, J=12.3 \mathrm{~Hz}, 1 \mathrm{H}\right.$ each, $\left.\mathrm{OCH}_{2} \mathrm{Ph}\right)$, $4.57\left(\mathrm{~d}, J=7.0 \mathrm{~Hz}, 1 \mathrm{H}, \mathrm{H}-1^{\mathrm{B}}\right), 4.41\left(\mathrm{~d}, J=7.6 \mathrm{~Hz}, 1 \mathrm{H}, \mathrm{H}-1^{\mathrm{F}}\right), 4.36,4.22(\mathrm{ABq}, J=11.8 \mathrm{~Hz}, 1 \mathrm{H}$ each, $\left.\mathrm{OCH}_{2} \mathrm{PMP}\right), 4.30\left(\mathrm{~s}, 1 \mathrm{H}, \mathrm{H}-4^{\mathrm{B}}\right), 4.17\left(\mathrm{ddd}, J=11.5,11.5,5.7 \mathrm{~Hz}, 1 \mathrm{H}, \mathrm{OCH} H \mathrm{CH}_{2} \mathrm{SiMe}_{3}\right), 4.09$ (dd, $J=$ 10.3, 8.8 Hz, $\left.1 \mathrm{H}, \mathrm{H}-2^{\mathrm{E}}\right), 4.06\left(\mathrm{~s}, 1 \mathrm{H}, \mathrm{H}-5^{\mathrm{F}}\right), 4.08-3.97\left(\mathrm{~m}, 4 \mathrm{H}, \mathrm{H}-2^{\mathrm{C}}, 2^{\mathrm{E}}, 4^{\mathrm{E}}, \mathrm{OCH} H \mathrm{CH}_{2} \mathrm{SiMe}_{3}\right), 3.95(\mathrm{~s}$, $\left.1 \mathrm{H}, \mathrm{H}-5^{\mathrm{B}}\right), 3.88-3.82\left(\mathrm{~m}, 1 \mathrm{H}, \mathrm{OCH} H \mathrm{CH}_{2} \mathrm{SiMe}_{3}\right), 3.81(\mathrm{~s}, 3 \mathrm{H}, \mathrm{OMe}), 3.69-3.64\left(\mathrm{~m}, 3 \mathrm{H}, \mathrm{H}-3^{\mathrm{B}}, 6^{\mathrm{E}}, 2^{\mathrm{F}}\right)$, $3.52-3.45\left(\mathrm{~m}, 3 \mathrm{H}, \mathrm{H}-5^{\mathrm{E}}, 6^{\mathrm{E}}, \mathrm{OCH} H \mathrm{CH}_{2} \mathrm{SiMe}_{3}\right), 3.42\left(\mathrm{dd}, J=9.1,9.1 \mathrm{~Hz}, 1 \mathrm{H}, \mathrm{H}-4^{\mathrm{C}}\right), 3.38-3.32(\mathrm{~m}, 1$ H, H-5 ${ }^{\mathrm{C}}$ ), 3.06 (s, $\left.1 \mathrm{H}, \mathrm{OH}\right), 1.84,1.72$ (s, $3 \mathrm{H}$ each, OAc), 1.29 (s, $\left.3 \mathrm{H}, \mathrm{Me}\right), 1.01$ (d, J=6.2 Hz, $3 \mathrm{H}, \mathrm{H}-$ $\left.6^{\mathrm{C}}\right), 0.92\left(\mathrm{ddd}, J=13.2,5.9 \mathrm{~Hz}, 1 \mathrm{H}, \mathrm{OCH}_{2} \mathrm{CH} H \mathrm{SiMe}_{3}\right), 0.88-0.76\left(\mathrm{~m}, 2 \mathrm{H}, \mathrm{OCH}_{2} \mathrm{CH}_{2} \mathrm{SiMe}_{3}\right), 0.74-$ $0.67\left(\mathrm{~m}, 1 \mathrm{H}, \mathrm{OCH}_{2} \mathrm{CH} H \mathrm{SiMe}_{3}\right),-0.00,-0.10$ (s, $9 \mathrm{H}$ each, $\left.\mathrm{SiMe}_{3}\right) ;{ }^{13} \mathrm{C} \mathrm{NMR}\left(125 \mathrm{MHz}, \mathrm{CDCl}_{3}\right): \delta \mathrm{ppm}$ $170.58,170.37,167.38,167.22,164.94,163.93,163.67,163.22,163.19,158.93,152.97,151.01,149.98$, $140.39,140.11,139.98,139.69,138.11,137.26,133.32,130.13,129.85,129.81,129.69,129.56,129.34$ $129.16,128.51,128.24,128.06,127.99,127.84,127.68,127.46,127.43,127.27,127.10,126.59,126.20$, $121.33,121.25,113.66,101.82,101.57,97.71,97.60,82.25,81.76,78.92,76.23,75.96,75.13,75.06$, $74.55,74.33,74.10,73.71,73.50,73.40,71.78,71.71,71.35,70.80,67.96,67.43,64.18,56.71,56.23$, $55.18,20.60,20.52,18.22,17.70,17.41,16.87,-1.41,-1.47$; LRMS calcd for $\mathrm{C}_{97} \mathrm{H}_{98} \mathrm{Cl}_{8} \mathrm{~N}_{2} \mathrm{O}_{30} \mathrm{Si}_{2} \mathrm{Na}[\mathrm{M}+$ $\mathrm{Na}]^{+}: 2129.3$, found 2129.4. 


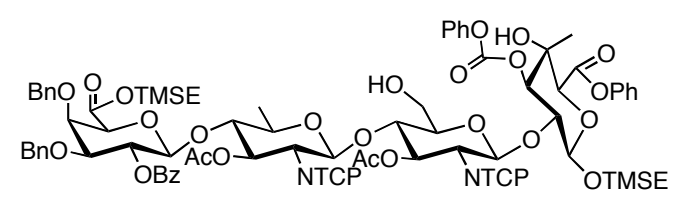

Phenyl \{[2-(Trimethylsilyl)ethyl 2- $O$-benzoyl-3,4-di- $O$-benzyl- $\beta$-D-galactopyranosyluronate]-(1 $\rightarrow 4)$ 3-O-acetyl-2,6-dideoxy-2-tetrachlorophthalimido- $\beta$-D-glucopyranosyl-(1 $\rightarrow$ 4)-3- $O$-acetyl-2-deoxy-2tetrachlorophthalimido- $\beta$-D-glucopyranosyl-(1 $\rightarrow 2)$-[2-(trimethylsilyl)ethyl 4-C-methyl-3-Ophenoxycarbonyl- $\beta$-D-glucopyranosid] $\}$ uronate (11).

The above compound $(112 \mathrm{mg}, 0.053 \mathrm{mmol})$ was dissolved in $\mathrm{CH}_{2} \mathrm{Cl}_{2}(2 \mathrm{~mL})$ and phosphate buffer $(\mathrm{pH}$ 7.0, $200 \mu \mathrm{L}$ ) then 2,3-dichloro-5,6-dicyano-1,4-benzoquinone (36 mg, $0.16 \mathrm{mmol}$ ) was added and the reaction was stirred at room temperature for $2 \mathrm{~h}$. The reaction was then diluted with $\mathrm{CH}_{2} \mathrm{Cl}_{2}$ and washed with saturated aqueous $\mathrm{NaHCO}_{3}, \mathrm{H}_{2} \mathrm{O}$, and brine. The organic phase was then dried over $\mathrm{Na}_{2} \mathrm{SO}_{4}$, concentrated in vacuo, then separated by silica gel chromatography (30\% EtOAc/petroleum ether) to afford the title compound (83 mg, $0.042 \mathrm{mmol}, 79 \%) .{ }^{1} \mathrm{H}$ NMR $\left(600 \mathrm{MHz}, \mathrm{CDCl}_{3}\right): \delta \mathrm{ppm} 7.95(\mathrm{~d}, J=$ $8.2 \mathrm{~Hz}, 2 \mathrm{H}, \mathrm{BzH}), 7.55$ (t, $J=7.6 \mathrm{~Hz}, 1 \mathrm{H}, \mathrm{BzH}), 7.41$ (t, $J=7.8 \mathrm{~Hz}, 2 \mathrm{H}, \mathrm{BzH}), 7.37$ (d, J= 7.9 Hz, $2 \mathrm{H}$, $\mathrm{PhH}), 7.33$ (t, $J=7.9 \mathrm{~Hz}, 2 \mathrm{H}, \mathrm{PhH}), 7.27-7.19(\mathrm{~m}, 8 \mathrm{H}, \mathrm{PhH}), 7.14$ (d, J= 7.6 Hz, $4 \mathrm{H}, \mathrm{PhH}), 7.09$ (d, $J$ $=7.6 \mathrm{~Hz}, 2 \mathrm{H}, \mathrm{PhH}), 7.07(\mathrm{~d}, J=7.6 \mathrm{~Hz}, 2 \mathrm{H}, \mathrm{PhH}), 5.57\left(\mathrm{dd}, J=10.2,8.2 \mathrm{~Hz}, 1 \mathrm{H}, \mathrm{H}-2^{\mathrm{B}}\right), 5.55-5.52$ $\left(\mathrm{m}, 3 \mathrm{H}, \mathrm{H}-3^{\mathrm{C}}, 1^{\mathrm{E}}, 3^{\mathrm{E}}\right), 5.44\left(\mathrm{~d}, J=8.5 \mathrm{~Hz}, 1 \mathrm{H}, \mathrm{H}-1^{\mathrm{C}}\right), 4.93,4.58\left(\mathrm{ABq}, J=12.0 \mathrm{~Hz}, 1 \mathrm{H}\right.$ each, $\left.\mathrm{OCH}_{2} \mathrm{Ph}\right)$, $4.83\left(\mathrm{~d}, J=9.9 \mathrm{~Hz}, 1 \mathrm{H}, \mathrm{H}-3^{\mathrm{F}}\right), 4.64,4.46\left(\mathrm{ABq}, J=12.3 \mathrm{~Hz}, 1 \mathrm{H}\right.$ each, $\left.\mathrm{OCH}_{2} \mathrm{Ph}\right), 4.56(\mathrm{~d}, J=7.9 \mathrm{~Hz}, 1$ $\left.\mathrm{H}, \mathrm{H}-1^{\mathrm{B}}\right), 4.45\left(\mathrm{~d}, J=7.6 \mathrm{~Hz}, 1 \mathrm{H}, \mathrm{H}-1^{\mathrm{F}}\right), 4.30\left(\mathrm{~d}, J=1.5 \mathrm{~Hz}, 1 \mathrm{H}, \mathrm{H}-4^{\mathrm{B}}\right), 4.20-4.15(\mathrm{~m}, 1 \mathrm{H}$, $\mathrm{OCH} H C H_{2} \mathrm{SiMe}_{3}$ ), 4.07 (s, $\left.1 \mathrm{H}, \mathrm{H}-5^{\mathrm{F}}\right), 4.08-3.94$ (m, 4H, H-2 $\left.{ }^{\mathrm{C}}, \mathrm{E}^{2}, \mathrm{OCH}_{2} \mathrm{CH}_{2} \mathrm{SiMe}_{3}\right), 3.95(\mathrm{~d}, J=1.1$ $\left.\mathrm{Hz}, 1 \mathrm{H}, \mathrm{H}-5^{\mathrm{B}}\right), 3.92\left(\mathrm{dd}, J=9.1,9.1 \mathrm{~Hz}, 1 \mathrm{H}, \mathrm{H}-4^{\mathrm{E}}\right), 3.66\left(\mathrm{dd}, J=9.3,2.9 \mathrm{~Hz}, 1 \mathrm{H}, \mathrm{H}-3^{\mathrm{B}}\right), 3.60-3.55(\mathrm{~m}$, $\left.3 \mathrm{H}, \mathrm{H}-6^{\mathrm{E}}, 2^{\mathrm{F}}, \mathrm{OCH} H \mathrm{CH}_{2} \mathrm{SiMe}_{3}\right), 3.45-3.42\left(\mathrm{~m}, 2 \mathrm{H}, \mathrm{H}-5^{\mathrm{E}}, 6^{\mathrm{E}}\right), 3.38-3.34(\mathrm{dd}, J=9.7,6.2 \mathrm{~Hz}, 1 \mathrm{H}, \mathrm{H}-$ $\left.5^{\mathrm{C}}\right), 3.33-3.29\left(\mathrm{~m}, 1 \mathrm{H}, \mathrm{H}-4^{\mathrm{C}}\right), 3.05(\mathrm{~s}, 1 \mathrm{H}, \mathrm{OH}), 1.87,1.66$ (s, $3 \mathrm{H}$ each, OAc), $1.26(\mathrm{~s}, 3 \mathrm{H}, \mathrm{Me}), 1.03$ $\left(\mathrm{d}, J=6.2 \mathrm{~Hz}, 3 \mathrm{H}, \mathrm{H}-6^{\mathrm{C}}\right), 1.00-0.81\left(\mathrm{~m}, 4 \mathrm{H}, 2 \times \mathrm{OCH}_{2} \mathrm{CH}_{2} \mathrm{SiMe}_{3}\right), 0.00,-0.02$ (s, $\left.9 \mathrm{H} \mathrm{each}, \mathrm{SiMe}_{3}\right)$; ${ }^{13} \mathrm{C}$ NMR (125 MHz, $\left.\mathrm{CDCl}_{3}\right)$ : $\delta \mathrm{ppm} 170.75,170.42,167.40,167.21,164.95,164.01,163.76,163.34$, $163.12,152.95,150.98,149.99,140.44,140.10,139.98,138.16,137.31,133.38,130.08,129.88,129.81$, 
$129.77,129.57,129.41,128.57,128.30,128.04,127.88,127.78,127.74,127.42,127.16,127.10,126.67$, $126.27,121.37,121.00,101.69,101.54,97.78,97.61,81.70,81.82,78.94,76.72,76.33,74.96,74.59$, $74.37,74.29,74.13,73.72,73.04,71.76,71.43,71.17,68.06,64.23,60.87,56.50,56.19,20.60,20.56$, 18.32, 17.89, 17.48, 16.85, -1.27, -1.42; LRMS calcd for $\mathrm{C}_{89} \mathrm{H}_{90} \mathrm{Cl}_{8} \mathrm{~N}_{2} \mathrm{O}_{29} \mathrm{Si}_{2} \mathrm{Na}[\mathrm{M}+\mathrm{Na}]^{+}: 2009.3$, found 2009.3.

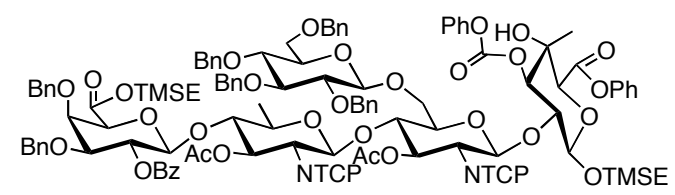

Phenyl \{[2-(Trimethylsilyl)ethyl 2- $O$-benzoyl-3,4-di- $O$-benzyl- $\beta$-D-galactopyranosyluronate]-(1 $\rightarrow 4)$ 3-O-acetyl-2,6-dideoxy-2-tetrachlorophthalimido- $\beta$-D-glucopyranosyl-(1 $\rightarrow 4)$-[2,3,4,6-tetra- $O$ -

benzyl- $\beta$-D-glucopyranosyl-(1 $\rightarrow 6)]-3-O$-acetyl-2-deoxy-2-tetrachlorophthalimido- $\beta$-D-

glucopyranosyl-( $1 \rightarrow 2)$-[2-(trimethylsilyl)ethyl 4-C-methyl-3- $O$-phenoxycarbonyl- $\beta$-Dglucopyranosid]\} uronate (12).

To a flame dried flask was added acceptor 11 (83 mg, $0.042 \mathrm{mmol})$, 4-allyl-1,2-dimethoxybenzene (144 $\mu \mathrm{L}, 0.87 \mathrm{mmol})$, and 2,6-di-tert-butyl-4-methylpyridine $(17 \mathrm{mg}, 0.083 \mathrm{mmol})$. The flask was placed under vacuum for $2 \mathrm{~h}$, switched to an argon atmosphere and $4 \AA$ molecular sieves were added followed by propionitrile $(5 \mathrm{~mL})$. The suspension was stirred at room temperature for $1 \mathrm{~h}$ then chilled to $-78{ }^{\circ} \mathrm{C}$ and treated with triflic anhydride $(10.5 \mathrm{~mL}, 0.063 \mathrm{mmol})$. A solution of phenyl 2,3,4,6-tetra-O-benzyl-1-thio$\beta$-D-glucopyranoside $S$-Oxide $(41 \mathrm{mg}, 0.063 \mathrm{mmol})$ in propionitrile $(1 \mathrm{~mL})$ was added dropwise by syringe pump over $15 \mathrm{~min}$. The reaction mixture was kept at $-78{ }^{\circ} \mathrm{C}$ for an additional 15 minutes then was quenched with triethylamine $(50 \mu \mathrm{L})$ and poured into a separatory funnel containing $\mathrm{H}_{2} \mathrm{O}$ and $\mathrm{CH}_{2} \mathrm{Cl}_{2}$. The organic phase then extracted from brine, dried over $\mathrm{Na}_{2} \mathrm{SO}_{4}$, and concentrated in vacuo. The crude residue was purified by silica gel chromatography $(20 \%$ EtOAc/petroleum ether) to afford the title compound (80 mg, $0.032 \mathrm{mmol}, 76 \%) .{ }^{1} \mathrm{H} \mathrm{NMR}\left(600 \mathrm{MHz}, \mathrm{CDCl}_{3}\right): \delta \mathrm{ppm} 7.99$ (dd, $J=8.2,1.2$ Hz, 2 H, BzH), 7.58 (t, J= 7.3 Hz, 1 H, BzH), $7.46-7.20$ (m, $31 \mathrm{H}, \mathrm{ArH}), 7.17$ (d, J=4.4 Hz, $4 \mathrm{H}, \mathrm{ArH})$, 
$7.17-7.14(\mathrm{~m}, 5 \mathrm{H}, \mathrm{ArH}), 7.04(\mathrm{dd}, J=8.6,1.0 \mathrm{~Hz}, 2 \mathrm{H}, \mathrm{ArH}), 5.66-5.56\left(\mathrm{~m}, 4 \mathrm{H}, \mathrm{H}-2^{\mathrm{B}}, 3^{\mathrm{C}}, 1^{\mathrm{E}}, 3^{\mathrm{E}}\right), 5.41$ $\left(\mathrm{d}, J=8.2 \mathrm{~Hz}, 1 \mathrm{H}, \mathrm{H}-1^{\mathrm{C}}\right), 4.98(\mathrm{~d}, J=11.7 \mathrm{~Hz}, 1 \mathrm{H}, \mathrm{OCH} H \mathrm{Ph}), 4.87(\mathrm{~d}, J=11.1 \mathrm{~Hz}, 1 \mathrm{H}, \mathrm{OCH} H \mathrm{Ph})$, $4.84\left(\mathrm{~d}, J=10.3 \mathrm{~Hz}, 1 \mathrm{H}, \mathrm{H}-3^{\mathrm{F}}\right), 4.81-4.74(\mathrm{~m}, 3 \mathrm{H}, \mathrm{OCH} H \mathrm{Ph}), 4.69(\mathrm{~d}, J=12.3 \mathrm{~Hz}, 1 \mathrm{H}, \mathrm{OCH} H \mathrm{Ph})$, 4.65 - 4.60 (m, 4 H, H-1 $\left.{ }^{\mathrm{B}}, \mathrm{OCH} H \mathrm{Ph}\right), 4.57$ - 4.52 (m, $\left.3 \mathrm{H}, \mathrm{H}-1^{\mathrm{D}}, \mathrm{OCH} H \mathrm{Ph}\right), 4.52-4.47$ (m, $2 \mathrm{H}, \mathrm{H}-1^{\mathrm{F}}$, OCH $H \mathrm{Ph}), 4.34\left(\mathrm{~d}, J=1.2 \mathrm{~Hz}, 1 \mathrm{H}, \mathrm{H}-4^{\mathrm{B}}\right), 4.26-4.18\left(\mathrm{~m}, 3 \mathrm{H}, \mathrm{H}-2^{\mathrm{E}}, 4^{\mathrm{E}}, \mathrm{OCH} H \mathrm{CH}_{2} \mathrm{SiMe}_{3}\right), 4.11(\mathrm{dd}, J=$ 10.4, 8.3 Hz, $\left.1 \mathrm{H}, \mathrm{H}-2^{\mathrm{C}}\right), 4.07\left(\mathrm{~s}, 1 \mathrm{H}, \mathrm{H}-5^{\mathrm{F}}\right), 4.08-4.02\left(\mathrm{~m}, 1 \mathrm{H}, \mathrm{OCH} H C H_{2} \mathrm{SiMe}_{3}\right), 3.98(\mathrm{~d}, J=1.2 \mathrm{~Hz}$,

$\left.1 \mathrm{H}, \mathrm{H}-5^{\mathrm{B}}\right), 3.98-3.91\left(\mathrm{~m}, 2 \mathrm{H}, \mathrm{H}-6, \mathrm{OCH} \mathrm{CH}_{2} \mathrm{SiMe}_{3}\right), 3.80-3.59\left(\mathrm{~m}, 8 \mathrm{H}, \mathrm{H}-3^{\mathrm{B}}, 3^{\mathrm{D}}, 4^{\mathrm{D}}, 5^{\mathrm{E}}, 6\right.$, $\mathrm{OCH} H C H_{2} \mathrm{SiMe}_{3}$ ), 3.52 - 3.45 (m, $3 \mathrm{H}, \mathrm{H}-4^{\mathrm{C}}, 5^{\mathrm{D}}, 6,3.44-3.38$ (m, $\left.1 \mathrm{H}, \mathrm{H}-5^{\mathrm{C}}\right), 3.09$ (s, $\left.1 \mathrm{H}, \mathrm{OH}\right), 2.93$ (dd, $J=8.5,8.5 \mathrm{~Hz}, 1 \mathrm{H}, \mathrm{H}-2^{\mathrm{D}}$ ), 1.90, 1.68 (s, $3 \mathrm{H}$ each, OAc), 1.28 (s, $\left.3 \mathrm{H}, \mathrm{Me}\right), 1.01$ (d, $J=5.9 \mathrm{~Hz}, 3 \mathrm{H}$, H-6 $\left.{ }^{\mathrm{C}}\right), 1.03-0.83\left(\mathrm{~m}, 4 \mathrm{H}, 2\right.$ x $\left.\mathrm{OCH}_{2} \mathrm{CH}_{2} \mathrm{SiMe}_{3}\right), 0.04,-0.06$ (s, $9 \mathrm{H}$ each, $\left.\mathrm{SiMe}_{3}\right) ;{ }^{13} \mathrm{C} \mathrm{NMR}(125 \mathrm{MHz}$, $\left.\mathrm{CDCl}_{3}\right): \delta$ ppm 170.69, 170.49, 167.41, 167.28, 164.96, 163.74, 163.46, 163.09, 163.06, 153.10, 151.02, $149.97,140.67,140.35,140.25,139.96,138.95,138.63,138.44,138.29,138.11,137.26,133.34,130.13$ $130.10,129.84,129.82,129.72,129.67,129.56,129.34,128.61,128.53,128.50,128.46,128.35,128.27$, $128.20,128.08,128.02,127.97,127.93,127.87,127.78,127.71,127.60,127.47,127.36,127.13,126.53$, $126.19,121.35,121.24,103.46,101.85,101.64,97.66,96.35,84.84,82.29,82.01,81.91,78.95,78.11$, $76.21,75.51,74.92,74.83,74.76,74.56,74.33,74.07,73.74,73.59,72.16,71.81,71.68,70.86,69.11$ $67.86,67.67,64.24,56.56,56.22,20.57,18.48,17.69,17.44,16.83,-1.32,-1.44$; LRMS calcd for $\mathrm{C}_{123} \mathrm{H}_{124} \mathrm{Cl}_{8} \mathrm{~N}_{2} \mathrm{O}_{34} \mathrm{Si}_{2} \mathrm{Na}[\mathrm{M}+\mathrm{Na}]^{+}:$2531.5, found 2531.4.

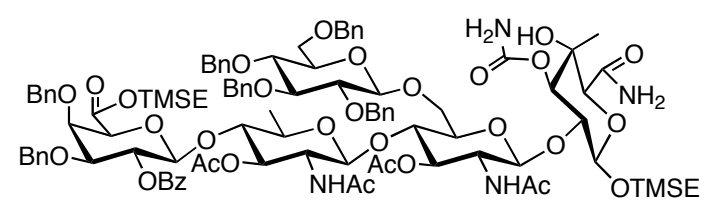

[2-(Trimethylsilyl)ethyl 2-O-Benzoyl-3,4-di- $O$-benzyl- $\beta$-D-galactopyranosyluronate]-(1 $\rightarrow 4)-2$ acetamido-2,6-dideoxy- $\beta$-D-glucopyranosyl-( $1 \rightarrow 4)$-[2,3,4,6-tetra- $O$-benzyl- $\beta$-D-glucopyranosyl$(1 \rightarrow 6)]-2$-acetamido-3- $O$-acetyl-2-deoxy- $\beta$-D-glucopyranosyl-(1 $\rightarrow 2)$-2-(trimethylsilyl)ethyl 3-Ocarbamoyl-4-C-methyl- $\beta$-D-glucopyranuronamide (13). 
To a solution of $12(80 \mathrm{mg}, 0.032 \mathrm{mmol})$ in $\mathrm{CH}_{2} \mathrm{Cl}_{2}(1 \mathrm{~mL})$ was added $\mathrm{NH}_{3}\left(5 \mathrm{~mL}\right.$ of $2 \mathrm{~N} \mathrm{NH}_{3}$ in isopropanol) and the reaction was stirred at room temperature for $1 \mathrm{~h}$. After concentration in vacuo, the crude residue was suspended in ethanol $(5 \mathrm{~mL})$ and ethylenediamine $(10.7 \mu \mathrm{L}, 0.160 \mathrm{mmol})$ was added. ${ }^{6}$ The reaction mixture was heated at $50{ }^{\circ} \mathrm{C}$ for $8.5 \mathrm{~h}$ then cooled to room temperature. An excess of acetic anhydride $(100 \mu \mathrm{L})$ was then added and the reaction was stirred for $15 \mathrm{~min}$ at room temperature before being concentrated in vacuo. The residue was then dissolved in pyridine $(3 \mathrm{~mL})$ and treated with acetic anhydride $(200 \mu \mathrm{L})$. After stirring at room temperature for $9 \mathrm{~h}$, the reaction was concentrated in vacuo then purified by silica gel chromatography $\left(5 \%\right.$ methanol $\left./ \mathrm{CH}_{2} \mathrm{Cl}_{2}\right)$ to afford the title compound (40 $\mathrm{mg}$, $0.021 \mathrm{mmol}, 66 \%) .{ }^{1} \mathrm{H} \mathrm{NMR}\left(600 \mathrm{MHz}, \mathrm{CDCl}_{3}\right): \delta \mathrm{ppm} 7.98(\mathrm{~d}, J=7.0 \mathrm{~Hz}, 2 \mathrm{H}), 7.58(\mathrm{t}, J=7.5 \mathrm{~Hz}, 1$ H), $7.44(\mathrm{t}, J=7.8 \mathrm{~Hz}, 2 \mathrm{H}), 7.35-7.18(\mathrm{~m}, 24 \mathrm{H}), 7.15(\mathrm{~d}, J=4.4 \mathrm{~Hz}, 4 \mathrm{H}), 7.13-7.08$ (m, $2 \mathrm{H}), 6.47$ (br s, $1 \mathrm{H}), 6.31$ (br s, $1 \mathrm{H}), 5.65($ br s, $1 \mathrm{H}), 5.62(\mathrm{~d}, J=9.1 \mathrm{~Hz}, 1 \mathrm{H}), 5.58(\mathrm{dd}, J=10.0,7.9 \mathrm{~Hz}, 1 \mathrm{H})$, $4.97(\mathrm{~d}, J=11.7 \mathrm{~Hz}, 1 \mathrm{H}), 4.87(\mathrm{~d}, J=11.1 \mathrm{~Hz}, 1 \mathrm{H}), 4.82(\mathrm{~d}, J=11.7 \mathrm{~Hz}, 1 \mathrm{H}), 4.77(\mathrm{~d}, J=10.8 \mathrm{~Hz}, 1$ H), $4.75-4.71(\mathrm{~m}, 3 \mathrm{H}), 4.68(\mathrm{~d}, J=5.9 \mathrm{~Hz}, 1 \mathrm{H}), 4.66(\mathrm{~d}, J=6.7 \mathrm{~Hz}, 1 \mathrm{H}), 4.62-4.56(\mathrm{~m}, 3 \mathrm{H}), 4.54$ $4.46(\mathrm{~m}, 5 \mathrm{H}), 4.32(\mathrm{br} \mathrm{s}, 1 \mathrm{H}), 4.30(\mathrm{~d}, J=7.9 \mathrm{~Hz}, 1 \mathrm{H}), 4.21(\mathrm{ddd}, J=11.6,11.6,5.6 \mathrm{~Hz}, 1 \mathrm{H}), 4.10(\mathrm{dd}$, $J=10.1,3.1 \mathrm{~Hz}, 1 \mathrm{H}), 4.09-4.01(\mathrm{~m}, 2 \mathrm{H}), 3.99-3.95(\mathrm{~m}, 2 \mathrm{H}), 3.92-3.80(\mathrm{~m}, 2 \mathrm{H}), 3.75-3.71(\mathrm{~m}, 2$ H), 3.70 - 3.59 (m, $6 \mathrm{H}), 3.59$ - $3.52(\mathrm{~m}, 3 \mathrm{H}), 3.51-3.47(\mathrm{~m}, 1 \mathrm{H}), 3.38(\mathrm{t}, J=8.5 \mathrm{~Hz}, 1 \mathrm{H}), 3.33(\mathrm{dd}, J=$ 8.8, 8.8 Hz, $1 \mathrm{H}), 3.03$ - $2.96(\mathrm{~m}, 1 \mathrm{H}), 2.06(\mathrm{~s}, 3 \mathrm{H}), 1.94$ (s, $3 \mathrm{H}), 1.90$ (s, $3 \mathrm{H}), 1.86$ (s, $3 \mathrm{H}), 1.08$ (s, 3 $\mathrm{H}), 1.03(\mathrm{~d}, J=6.2 \mathrm{~Hz}, 3 \mathrm{H}), 1.01-0.85(\mathrm{~m}, 4 \mathrm{H}), 0.02(\mathrm{~s}, 9 \mathrm{H}),-0.00(\mathrm{~s}, 9 \mathrm{H}) ; ;{ }^{13} \mathrm{C} \mathrm{NMR}(100 \mathrm{MHz}$, $\left.\mathrm{CDCl}_{3}\right): \delta \mathrm{ppm} 171.72,171.55,170.97,170.78,170.32,167.42,164.98,157.20,138.65,138.22,138.19$, $138.01,137.28,133.45,129.84,129.77,128.72,128.64,128.55,128.50,128.29,128.20,128.01,128.00$, $127.96,127.86,127.82,127.77,127.73,103.51,102.10,101.53,100.93,100.80,84.77,82.29,80.88$, $79.88,78.97,77.99,75.89,75.68,75.57,75.07,74.85,74.69,74.60,74.31,74.26,73.50,73.40,72.78$, $71.80,71.73,70.89,68.95,68.49,68.31,64.14,54.55,53.06,23.57,23.26,21.02,20.92,18.58,17.80$, 17.56, 16.88, -1.19, -1.42; LRMS calcd for $\mathrm{C}_{99} \mathrm{H}_{126} \mathrm{~N}_{4} \mathrm{O}_{30} \mathrm{Si}_{2}[\mathrm{M}+\mathrm{H}]^{+}:$1907.8, found 1907.7. 


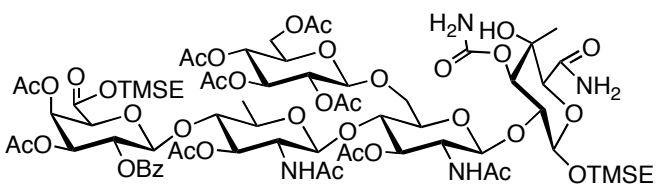

[2-(Trimethylsilyl)ethyl 3,4-Di-O-acetyl-2-O-benzoyl- $\beta$-D-galactopyranosyluronate]-( $1 \rightarrow 4)$-2-

acetamido-3- $O$-acetyl-2,6-dideoxy- $\beta$-D-glucopyranosyl-(1 $\rightarrow 4)-[2,3,4,6$-tetra- $O$-acetyl- $\beta$-D-

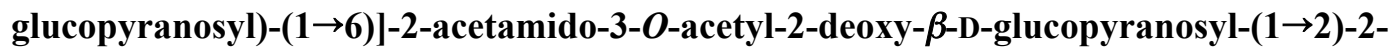

(trimethylsilyl)ethyl 3-O-carbamoyl-4-C-methyl- $\beta$-D-glucopyranuronamide (14).

To a stirred solution of $13(51 \mathrm{mg}, 0.027 \mathrm{mmol})$ in degassed methanol $(25 \mathrm{~mL})$ under an atmosphere of argon was added $\mathrm{Pd}(\mathrm{OH})_{2} / \mathrm{C}(20 \mathrm{mg})$. The suspension was degassed and backfilled with $\mathrm{H}_{2}$ three times then stirred under $\mathrm{H}_{2}(1 \mathrm{~atm})$ for $11 \mathrm{~h}$. The reaction was then filtered through a pad of celite and concentrated in vacuo. The crude residue was then dissolved in pyridine $(2 \mathrm{~mL})$ and treated with acetic anhydride $(200 \mu \mathrm{L})$. After stirring at room temperature for $9 \mathrm{~h}$, the reaction mixture was concentrated the separated by silica gel chromatography $\left(7 \%\right.$ methanol/ $\left./ \mathrm{CH}_{2} \mathrm{Cl}_{2}\right)$ to afford the title compound $(28 \mathrm{mg}, 0.017$ mmol, 63\%). ${ }^{1} \mathrm{H}$ NMR (500 MHz, $\left.\mathrm{CDCl}_{3}\right): \delta \mathrm{ppm} 7.97(\mathrm{~d}, J=6.8 \mathrm{~Hz}, 2 \mathrm{H}, \mathrm{PhH}), 7.59$ (t, $J=7.3 \mathrm{~Hz}, 1$ H, PhH), $7.46(\mathrm{t}, J=7.8 \mathrm{~Hz}, 2 \mathrm{H}, \mathrm{PhH}), 6.95,5.90$ (br s, $1 \mathrm{H}$ each, $\mathrm{NHH}), 6.28(\mathrm{~d}, J=8.8 \mathrm{~Hz}, 1 \mathrm{H}$, $\left.\mathrm{NHAc}^{\mathrm{E}}\right), 5.98\left(\mathrm{~d}, J=8.8 \mathrm{~Hz}, 1 \mathrm{H}, \mathrm{NHAc}^{\mathrm{C}}\right), 5.70\left(\mathrm{~d}, J=2.0 \mathrm{~Hz}, 1 \mathrm{H}, \mathrm{H}-4^{\mathrm{B}}\right), 5.43(\mathrm{dd}, J=10.3,7.8 \mathrm{~Hz}, 1$ $\left.\mathrm{H}, \mathrm{H}-2^{\mathrm{B}}\right), 5.31\left(\mathrm{dd}, J=9.3,9.3 \mathrm{~Hz}, 1 \mathrm{H}, \mathrm{H}-3^{\mathrm{D}}\right), 5.25\left(\mathrm{dd}, J=10.3,3.4 \mathrm{~Hz}, 1 \mathrm{H}, \mathrm{H}-3^{\mathrm{B}}\right), 5.00-5.07(\mathrm{~m}, 2$ $\left.\mathrm{H}, \mathrm{H}-3^{\mathrm{C}}, 4^{\mathrm{D}}\right), 4.95\left(\mathrm{dd}, J=9.3,7.8 \mathrm{~Hz}, 1 \mathrm{H}, \mathrm{H}-2^{\mathrm{D}}\right), 4.90-4.80\left(\mathrm{~m}, 3 \mathrm{H}, \mathrm{H}-1^{\mathrm{D}}, \mathrm{E}^{3}, \mathrm{~F}^{3}\right), 4.76(\mathrm{br} \mathrm{s}, 1 \mathrm{H}), 4.72$ $\left(\mathrm{d}, J=7.8 \mathrm{~Hz}, 1 \mathrm{H}, \mathrm{H}-1^{\mathrm{B}}\right), 4.55\left(\mathrm{~d}, J=7.3 \mathrm{~Hz}, 1 \mathrm{H}, \mathrm{H}-1^{\mathrm{E}}\right), 4.46\left(\mathrm{~d}, J=7.3 \mathrm{~Hz}, 1 \mathrm{H}, \mathrm{H}-1^{\mathrm{F}}\right), 4.29(\mathrm{~s}, 1 \mathrm{H}, \mathrm{H}-$ $\left.5^{\mathrm{B}}\right), 4.28-4.23\left(\mathrm{~m}, 2 \mathrm{H}, \mathrm{H}-1^{\mathrm{C}}, \mathrm{OCH} H C H_{2} \mathrm{SiMe}_{3}\right), 4.23-4.18\left(\mathrm{~m}, 2 \mathrm{H}, \mathrm{H}-6^{\mathrm{D}}, \mathrm{OCH} H \mathrm{CH}_{2} \mathrm{SiMe}_{3}\right), 4.15(\mathrm{~d}$, $\left.J=10.7 \mathrm{~Hz}, 1 \mathrm{H}, \mathrm{H}-6^{\mathrm{D}}\right), 4.06-3.99\left(\mathrm{~m}, 1 \mathrm{H}, \mathrm{H}-2^{\mathrm{E}}\right), 3.94(\mathrm{ddd}, J=16.4,5.9,5.6 \mathrm{~Hz}, 1 \mathrm{H}$, $\left.\mathrm{OCH} H C H_{2} \mathrm{SiMe}_{3}\right), 3.86\left(\mathrm{~s}, 1 \mathrm{H}, \mathrm{H}-5^{\mathrm{F}}\right), 3.90-3.81\left(\mathrm{~m}, 2 \mathrm{H}, \mathrm{H}-2^{\mathrm{C}}, 6^{\mathrm{E}}\right), 3.76-3.68\left(\mathrm{~m}, 3 \mathrm{H}, \mathrm{H}-5^{\mathrm{D}}, 6^{\mathrm{E}}\right.$, $\left.\mathrm{OCH} H \mathrm{CH}_{2} \mathrm{SiMe}_{3}\right), 3.59-3.52\left(\mathrm{~m}, 3 \mathrm{H}, \mathrm{H}-4^{\mathrm{E}}, 5^{\mathrm{E}}, 2^{\mathrm{F}}\right), 3.42\left(\mathrm{dd}, J=9.0,9.0 \mathrm{~Hz}, 1 \mathrm{H}, \mathrm{H}-4^{\mathrm{C}}\right), 3.29(\mathrm{dd}, J=$ 8.8, 6.2 Hz, $\left.1 \mathrm{H},{\mathrm{H}-5^{\mathrm{C}}}^{\mathrm{C}}\right), 2.12,2.11,2.07,2.06,2.01,1.97,1.95,1.92,1.89,1.87$, (s, $3 \mathrm{H}$ each, 2 x NHAc, $8 \times \mathrm{OAc}) 1.17(\mathrm{~s}, 3 \mathrm{H}, \mathrm{Me}), 1.09\left(\mathrm{~d}, J=6.3 \mathrm{~Hz}, 3 \mathrm{H}, \mathrm{H}-6^{\mathrm{C}}\right), 1.06-0.91\left(\mathrm{~m}, 4 \mathrm{H}, 2 \times \mathrm{OCH}_{2} \mathrm{CH}_{2} \mathrm{SiMe}_{3}\right)$, 0.04, 0.03 (s, $9 \mathrm{H}$ each, $\left.\mathrm{SiMe}_{3}\right) ;{ }^{13} \mathrm{C}$ NMR (125 MHz, $\left.\mathrm{CD}_{3} \mathrm{OD}\right): \delta \mathrm{ppm} 173.86,173.79,173.59,172.82$, 
$172.29,171.91,171.63,171.46,171.33,171.21,167.78,166.52,158.98,135.01,130.90,130.65,129.98$, $103.02,102.27,102.24,102.05,101.64,82.99,81.09,78.03,77.77,77.36,75.40,74.19,74.14,74.08$ $73.65,73.03,72.70,72.15,72.07,71.20,70.70,70.32,69.33,68.81,65.21,63.29,56.21,55.70,23.19$, $23.15,21.44,21.35,21.27,20.89,20.85,20.70,20.62,20.54,19.60,18.55,18.39,17.40,-1.10,-1.43$; LRMS calcd for $\mathrm{C}_{69} \mathrm{H}_{104} \mathrm{~N}_{4} \mathrm{O}_{36} \mathrm{Si}_{2}[\mathrm{M}+\mathrm{H}]^{+}:$1619.6, found 1619.5.

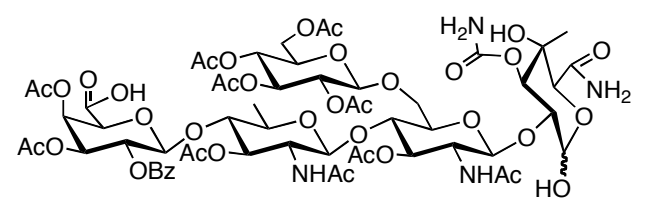

3,4-Di- $O$-acetyl-2- $O$-benzoyl- $\beta$-D-galactopyranuronic acid-( $1 \rightarrow 4)$-2-acetamido-3- $O$-acetyl-2-deoxy-

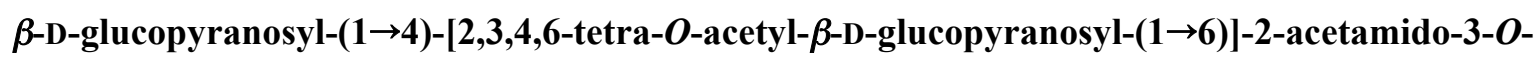
acetyl-2-deoxy- $\beta$-D-glucopyranosyl)-(1 $\rightarrow 2)-3$-carbamoyl-4-C-methyl- $\alpha / \beta-D-$ glucopyranuronamide.

To a solution of compound $\mathbf{1 4}(20.2 \mathrm{mg}, 12.5 \mu \mathrm{mol})$ in $\mathrm{CH}_{2} \mathrm{Cl}_{2}(1 \mathrm{ml}), \mathrm{BF}_{3} \cdot \mathrm{OEt}_{2}(20 \mu \mathrm{l})$ was added dropwise at room temperature. ${ }^{3}$ After stirring for $1 \mathrm{~h}$, the mixture was directly applied onto a short silica column. The product was eluted with $\mathrm{CHCl}_{3} / \mathrm{MeOH} / \mathrm{H}_{2} \mathrm{O}(18 / 12 / 2.7)$ to afford the title compound (17.0 mg, $12.0 \mu \mathrm{mol}, 97 \%) .{ }^{1} \mathrm{H}$ NMR (600 MHz, $\left.\mathrm{D}_{2} \mathrm{O}\right) \delta 8.00$ - 7.42 (m, $\left.5 \mathrm{H}, \mathrm{PhH}\right), 5.59(\mathrm{~m}, 1 \mathrm{H}), 5.38$ - 5.28 (m, 2 H), 5.22 - $5.14(\mathrm{~m}, 2 \mathrm{H}), 4.99-4.78(\mathrm{~m}, 6 \mathrm{H}), 4.54-4.50(\mathrm{~m}, 1 \mathrm{H}), 4.44(\mathrm{t}, \mathrm{J}=8.5 \mathrm{~Hz}, 1 \mathrm{H}), 4.30$ $4.24(\mathrm{~m}, 1 \mathrm{H}), 4.20(\mathrm{~s}, 1 \mathrm{H}), 3.92(\mathrm{~s}, 1 \mathrm{H}), 3.87$ - $3.84(\mathrm{~m}, 1 \mathrm{H}), 3.80$ - $3.48(\mathrm{~m}, 8 \mathrm{H}), 3.25(\mathrm{~m}, 1 \mathrm{H}) 2.09$ $1.70(\mathrm{~m}, 30 \mathrm{H}), 1.09(\mathrm{~s}, 3 \mathrm{H}), 0.91-0.88(\mathrm{~m}, 3 \mathrm{H})$; LRMS calcd for $\mathrm{C}_{59} \mathrm{H}_{79} \mathrm{~N}_{4} \mathrm{O}_{36}[\mathrm{M}+\mathrm{H}]^{+}:$: 1419.4, found 1419.4 .

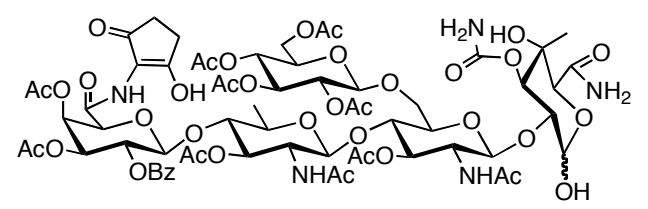

5-N-(3-hydroxy-1-oxocyclopenten-2-yl)-3,4-di- $O$-acetyl-2- $O$-benzoyl- $\beta$-D-galactopyranuronamide$(1 \rightarrow 4)$-2-acetamido-3- $O$-acetyl-2-deoxy- $\beta$-D-glucopyranosyl-( $1 \rightarrow 4)$-[2,3,4,6-tetra- $O$-acetyl- $\beta$-D- 
glucopyranosyl-(1 $\rightarrow 6)]$-2-acetamido-3- $O$-acetyl-2-deoxy- $\beta$-D-glucopyranosyl-(1 $\rightarrow 2)$-3-carbamoyl-4C-methyl- $\alpha / \beta$-D-glucopyranuronamide.

To a solution of the above compound $(9.0 \mathrm{mg}, 6.3 \mu \mathrm{mol})$ and HATU $(9.9 \mathrm{mg}, 26.0 \mu \mathrm{mol})$ in $\mathrm{CH}_{2} \mathrm{Cl}_{2} / \mathrm{DMF}$ (2/1, $0.5 \mathrm{ml})$, a solution of DIPEA $\left(1.1 \mu \mathrm{L}\right.$ in $\left.40 \mu \mathrm{L} \mathrm{CH}_{2} \mathrm{Cl}_{2}, 6.3 \mu \mathrm{mol}\right)$ was added dropwise. After the mixture was stirred for $10 \mathrm{~min}$, a solution of 2-amino-3-hydroxy-2-cyclopenten-1-one hydrochloride (4.8 $\mathrm{mg}, 32.0 \mu \mathrm{mol})$ in DMF $(0.5 \mathrm{ml})$ was added followed by the slow addition of a solution of DIPEA (4.4 $\mu \mathrm{L}$ in $\left.160 \mu \mathrm{L} \mathrm{CH}_{2} \mathrm{Cl}_{2}, 25.2 \mu \mathrm{mol}\right)$ ) over $3 \mathrm{~h}$. After concentration in vacuo, the black mixture was purified by silica gel chromatography $\mathrm{CHCl}_{3} / \mathrm{MeOH} / \mathrm{H}_{2} \mathrm{O}(18 / 12 / 2.7)$ to give a semi-pure product. Further purification by semi-prep HPLC, using a $0-100 \%$ gradient of $\mathrm{CH}_{3} \mathrm{CN} / \mathrm{H}_{2} \mathrm{O}$ with $0.1 \%$ TFA, afforded the title compound $(5.2 \mathrm{mg}, 55 \%)$ as a mixture $\left(\alpha: \beta, 2: 1\right.$ initial ratio becomes $5: 1$ after $40 \mathrm{~h}$ of sitting in $\mathrm{D}_{2} \mathrm{O}$ solution). ${ }^{1} \mathrm{H}$ NMR (600 MHz, $\left.\mathrm{D}_{2} \mathrm{O}\right) \delta 8.10-7.60(\mathrm{PhH}, 5 \mathrm{H}), 5.80\left(\right.$ br d, $\left.J=2.3 \mathrm{~Hz}, 1 \mathrm{H}, \mathrm{H}-4^{\mathrm{B}}\right), 5.53$ $(\mathrm{dd}, J=3.5,10.6 \mathrm{~Hz}, 1 \mathrm{H}), 5.49\left(\mathrm{~d}, J=3.8 \mathrm{~Hz}, 1 \mathrm{H}, \mathrm{H}-1^{\mathrm{F}}\right), 5.43(\mathrm{t}, J=8.6 \mathrm{~Hz}, 0.2 \mathrm{H}), 5.35(\mathrm{dd}, J=7.9$, $9.7 \mathrm{~Hz}, 1 \mathrm{H}), 5.33(\mathrm{t}, J=9.4 \mathrm{~Hz}, 1 \mathrm{H}), 5.25(\mathrm{~m}, 1 \mathrm{H}), 5.13-5.08(\mathrm{~m}, 2.2 \mathrm{H}), 5.04(\mathrm{dd}, \mathrm{J}=8.5,10.2 \mathrm{~Hz}, 1$ H), $5.01\left(\mathrm{~d}, J=10.8 \mathrm{~Hz}, 1 \mathrm{H}, \mathrm{H}-3^{\mathrm{F}}\right), 4.93(\mathrm{dd}, J=7.9,9.3 \mathrm{~Hz}, 1 \mathrm{H}), 4.84(\mathrm{~d}, J=7.7 \mathrm{~Hz}, 1 \mathrm{H}), 4.77(\mathrm{~s}, 1$ H, H-5 $\left.5^{\mathrm{B}}\right), 4.67-4.65(\mathrm{~m}, 1.6 \mathrm{H}), 4.60-4.59(\mathrm{~m}, 1.2 \mathrm{H}), 4.44\left(\mathrm{~s}, 1 \mathrm{H}, \mathrm{H}-5^{\mathrm{F}}\right), 4.40-4.38$ (m, $\left.1.2 \mathrm{H}\right), 4.22$ (br d, $J=10.8 \mathrm{~Hz}, 1 \mathrm{H}), 4.05(\mathrm{~s}, 0.2 \mathrm{H}), 4.00-3.97$ (m, $2 \mathrm{H}), 3.86-3.63(\mathrm{~m}, 10.2 \mathrm{H}), 3.35(\mathrm{~m}, 1 \mathrm{H}), 2.65$ $\left(\mathrm{s}, 4 \mathrm{H}, \mathrm{H}^{\mathrm{A}}\right), 2.19-1.87(\mathrm{~m}, 36.8 \mathrm{H}), 1.22(\mathrm{~s}, 3 \mathrm{H}), 1.09$ and $1.07\left(2 \mathrm{~d}, \mathrm{~J}=6.2 \mathrm{~Hz}, 3 \mathrm{H}, \mathrm{H}-6{ }^{\mathrm{C}}\right) ;{ }^{13} \mathrm{C}$ NMR (125 MHz, $\left.\mathrm{D}_{2} \mathrm{O}\right) \delta 197.5,177.1,176.9,176.5,176.1,176.0,175.8,175.7,176.6,175.5,175.3,175.2$, $170.0,169.8,160.9,137.4 .132 .5,131.7,130.7,114.3,104.0,103.0,102.7,102.6,94.2,82.7,80.2,78.2$, $77.2,76.6,76.0,75.9,75.7,75.6,75.5,74.4,64.3,74.2,73.8,73.1,72.7,70.9,70.6,64.3,56.9,56.2,31.2$, 24.4, 24.3, 22.7, 22.6, 22.5, 22.4, 22.3, 22.2, 19.3, 19.2, 17.0; LRMS calcd for $\mathrm{C}_{64} \mathrm{H}_{83} \mathrm{~N}_{5} \mathrm{O}_{37} \mathrm{Na}[\mathrm{M}+\mathrm{Na}]^{+}$: 1536.4, found 1536.4 .

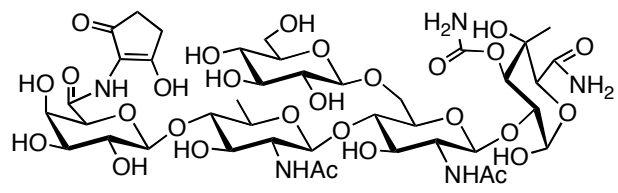


5-N-(3-hydroxy-1-oxocyclopenten-2-yl)- $\beta$-D-galactopyranuronamide-(1 $\rightarrow 4)-2$-acetamido-2-deoxy- $\beta$ -

D-glucopyranosyl-(1 $\rightarrow 4)$-[ $\beta$-D-glucopyranosyl-(1 $\rightarrow 6)]$-2-acetamido-2-deoxy- $\beta$-D-glucopyranosyl(1 $\rightarrow 2)$-3-carbamoyl-4-C-methyl- $\alpha$-D-glucopyranuronamide (15).

Two methods for preparation:

Method 1: To a solution of the above compound $(10.6 \mathrm{mg}, 7.0 \mu \mathrm{mol})$ in $\mathrm{MeOH}(0 \mu \mathrm{l})$ was added a solution of $1 \mathrm{~N} \mathrm{NaOH}(70 \mu \mathrm{L}, 70.0 \mu \mathrm{mol})$. After stirring for $1 \mathrm{~h}$, the mixture was directly injected onto a semi-prep reversed-phase HPLC, using a $0-100 \%$ gradient of $\mathrm{CH}_{3} \mathrm{CN} / \mathrm{H}_{2} \mathrm{O}$ with $0.1 \%$ TFA, to afford the title compound $(6.0 \mathrm{mg}, 5.6 \mu \mathrm{mol}, 80 \%)$. Apparently, treatment of the $\alpha: \beta(5: 1)$ starting material mixture with $\mathrm{NaOH}$ facilitated conversion of the minor $\beta$ isomer to the $\alpha$ product, affording only the $\alpha$ isomer after purification; HRMS calcd for $\mathrm{C}_{41} \mathrm{H}_{64} \mathrm{~N}_{5} \mathrm{O}_{28}[\mathrm{M}+\mathrm{H}]^{+}$: 1074.3738, found 1074.3723.

Method 2: To a solution of $\mathbf{1 6}(5.0 \mathrm{mg}, 3.4 \mu \mathrm{mol})$, obtained from the degradation of moenomycin $\mathrm{A}^{7}$, in MeOH $(700 \mu \mathrm{l})$, was added a solution of $1 \mathrm{~N} \mathrm{NaOH}(70 \mu \mathrm{L})$. The mixture was stirred for 45 min then directly injected onto a semi-prep reversed-phase HPLC, using a $0-100 \%$ gradient of $\mathrm{CH}_{3} \mathrm{CN} / \mathrm{H}_{2} \mathrm{O}$ with $0.1 \%$ TFA to afford the title compound as the $\alpha$ product $(2.6 \mathrm{mg}, 2.4 \mu \mathrm{mol}, 70 \%)$. LRMS calcd for $\mathrm{C}_{41} \mathrm{H}_{64} \mathrm{~N}_{5} \mathrm{O}_{28}[\mathrm{M}+\mathrm{H}]^{+}:$1074.4, found 1074.3.

NMR spectra of product identical for both methods: ${ }^{1} \mathrm{H}$ NMR $\left(600 \mathrm{MHz}, \mathrm{D}_{2} \mathrm{O}\right) \delta 5.54(\mathrm{~d}, J=3.8 \mathrm{~Hz}, 1$ $\left.\mathrm{H}, \mathrm{H}-1^{\mathrm{F}}\right), 5.01\left(\mathrm{~d}, J=10.5 \mathrm{~Hz}, 1 \mathrm{H}, \mathrm{H}-3^{\mathrm{F}}\right), 4.65\left(\mathrm{~d}, J=7.9 \mathrm{~Hz}, 1 \mathrm{H}, \mathrm{H}-1^{\mathrm{B}}\right), 4.59\left(\mathrm{~d}, J=8.5 \mathrm{~Hz}, 1 \mathrm{H}, \mathrm{H}-1^{\mathrm{E}}\right)$, $4.55\left(\mathrm{~d}, J=8.5 \mathrm{~Hz}, 1 \mathrm{H}, \mathrm{H}-1^{\mathrm{C}}\right), 4.46\left(\mathrm{~d}, J=8.0 \mathrm{~Hz}, 1 \mathrm{H}, \mathrm{H}-1^{\mathrm{D}}\right), 4.45\left(\mathrm{~s}, 1 \mathrm{H}, \mathrm{H}-5^{\mathrm{B}}\right), 4.38\left(\mathrm{~s}, 1 \mathrm{H}, \mathrm{H}-5^{\mathrm{F}}\right)$, $4.28\left(\right.$ br d, $\left.J=3.5 \mathrm{~Hz}, 1 \mathrm{H}, \mathrm{H}-4^{\mathrm{B}}\right), 4.17(\mathrm{~d}, J=10.5 \mathrm{~Hz}, 1 \mathrm{H}), 3.98(\mathrm{dd}, J=12.3,2.1 \mathrm{~Hz}, 1 \mathrm{H}), 3.79-3.41$ (m, $16 \mathrm{H}), 3.33\left(\mathrm{dd}, J=9.4,8.0 \mathrm{~Hz}, 1 \mathrm{H}, \mathrm{H}-2^{\mathrm{D}}\right), 2.63\left(\mathrm{~s}, 4 \mathrm{H}, \mathrm{H}-3^{\mathrm{A}}\right), 1.07,1.05$ (s, $3 \mathrm{H}$ each, NHAc), 1.39 $\left(\mathrm{d}, J=5.8 \mathrm{~Hz}, 3 \mathrm{H}, \mathrm{H}-6^{\mathrm{C}}\right), 1.21\left(\mathrm{~s}, 3 \mathrm{H}, \mathrm{CH}_{3}\right) ;{ }^{13} \mathrm{C} \mathrm{NMR}\left(125 \mathrm{MHz}, \mathrm{D}_{2} \mathrm{O}\right) \delta 197.2,177.4,176.0,172.5$,

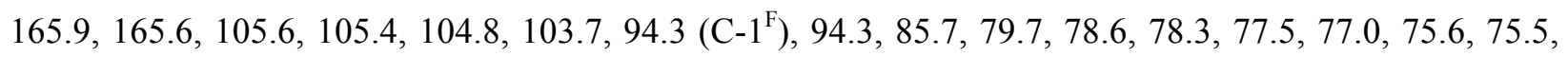
$74.8,74.6,74.4,74.2,73.6,73.1,72.0,71.4,70.6,63.2,58.1,57.4,31.1,24.7,24.6,19.0,16.9$. 


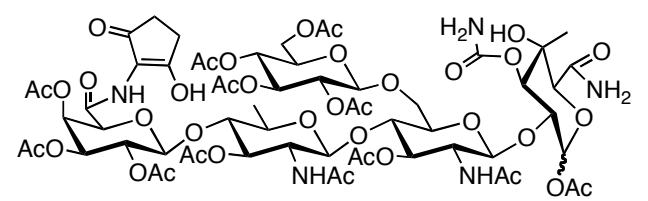

5-N-(3-hydroxy-1-oxocyclopenten-2-yl)-3,4-di- $O$-acetyl-2-O-benzoyl- $\beta$-D-galactopyranuronamide$(1 \rightarrow 4)$-2-acetamido-3- $O$-acetyl-2-deoxy- $\beta$-D-glucopyranosyl-( $1 \rightarrow 4)$-[2,3,4,6-tetra- $O$-acetyl- $\beta$-D-

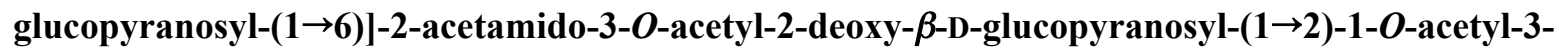
carbamoyl-4-C-methyl- $\alpha$-D-glucopyranuronamide.

Compound 15 (4.5 mg, $4.19 \mu \mathrm{mol})$ in $\mathrm{Ac}_{2} \mathrm{O} /$ pyridine $(1 \mathrm{ml} / 1 \mathrm{ml})$ was stirred overnight. After evaporation of solvents and coevaporation with $\mathrm{MeOH}$ twice, the product was purified by gradient reversed-phase chromatography, using a $0-100 \%$ gradient of $\mathrm{CH}_{3} \mathrm{CN} / \mathrm{H}_{2} \mathrm{O}$, to afford the title compound $(4.8 \mathrm{mg}, 3.27$ umol, 78\%). ${ }^{1} \mathrm{H}$ NMR (600 MHz, $\left.\mathrm{D}_{2} \mathrm{O}\right) \delta 6.45\left(\mathrm{~d}, J=3.8 \mathrm{~Hz}, 1 \mathrm{H}, \mathrm{H}-1^{\mathrm{F}}\right), 4.72\left(\mathrm{br} \mathrm{s}, 1 \mathrm{H}, \mathrm{H}-4^{\mathrm{B}}\right), 5.45(\mathrm{t}, J$ $\left.=9.7 \mathrm{~Hz}, 1 \mathrm{H}, \mathrm{H}-3^{\mathrm{D}}\right), 5.30\left(\mathrm{dd}, J=3.3,11.0 \mathrm{~Hz}, 1 \mathrm{H}, \mathrm{H}-3^{\mathrm{B}}\right), 5.15-5.03(\mathrm{~m}, 6 \mathrm{H}), 4.98(\mathrm{t}, J=8.2 \mathrm{~Hz}, 1$ H), $4.88\left(\mathrm{~d}, J=7.9 \mathrm{~Hz}, 1 \mathrm{H}, \mathrm{H}-1^{\mathrm{D}}\right), 4.75\left(\mathrm{~d}, J=8.8 \mathrm{~Hz}, 1 \mathrm{H}, \mathrm{H}-1^{\mathrm{C}}\right), 4.70\left(\mathrm{~s}, 1 \mathrm{H}, \mathrm{H}-5^{\mathrm{B}}\right), 4.69(\mathrm{~d}, J=8.0$ $\left.\mathrm{Hz}, 1 \mathrm{H}, \mathrm{H}-1^{\mathrm{E}}\right), 4.41(\mathrm{dd}, J=11.8,3.0 \mathrm{~Hz}, 1 \mathrm{H}), 4.39\left(\mathrm{~s}, 1 \mathrm{H}, \mathrm{H}-5^{\mathrm{F}}\right), 4.27(\mathrm{br} \mathrm{d}, J=11.8 \mathrm{~Hz}, 1 \mathrm{H}), 4.01-$ $3.92(\mathrm{~m}, 3 \mathrm{H}), 3.84-3.76(\mathrm{~m}, 5 \mathrm{H}), 3.70(\mathrm{~m}, 1 \mathrm{H}), 3.61(\mathrm{~m}, 1 \mathrm{H}), 2.64\left(\mathrm{~s}, 4 \mathrm{H}, \mathrm{H}^{\mathrm{A}}\right), 2.25-1.96(\mathrm{~m}, 36 \mathrm{H}$, $12 \times \mathrm{Ac}), 1.28\left(\mathrm{~d}, J=6.6 \mathrm{~Hz}, 3 \mathrm{H}, \mathrm{H}-6^{\mathrm{C}}\right), 1.25$ (s, $\left.3 \mathrm{H}, \mathrm{Me}\right) ;{ }^{13} \mathrm{C} \mathrm{NMR}\left(125 \mathrm{MHz}, \mathrm{D}_{2} \mathrm{O}\right) \delta 197.5,179.5$, $177.0,176.5,176.2,175.8,175.7,175.6,175.4,175.1,169.8,166.1,165.8,160.9,114.3,104.3,103.3$ $103.2,102.5,93.0,82.6,78.8,77.0,76.6,76.2,76.1,75.9,75.6,75.4,75.3,74.5,73.9,73.3,73.2,72.3$, $71.5,70.8,64.5,57.1,56.3,31.2,24.5,22.9,22.8,22.6,22.4,22.3,22.2,19.3,17.2$; LRMS calcd for $\mathrm{C}_{61} \mathrm{H}_{84} \mathrm{~N}_{5} \mathrm{O}_{38}[\mathrm{M}+\mathrm{H}]^{+}:$1494.4, found 1494.4.

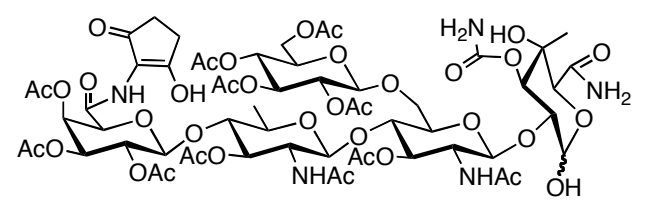

5-N-(3-hydroxy-1-oxocyclopenten-2-yl)-2,3,4-tri- $O$-acetyl- $\beta$-D-galactopyranuronamide-(1 $\rightarrow 4)-2$ acetamido-3- $O$-acetyl-2-deoxy- $\beta$-D-glucopyranosyl-(1 $\rightarrow 4)$-[2,3,4,6-tetra- $O$-acetyl- $\beta$-D- 


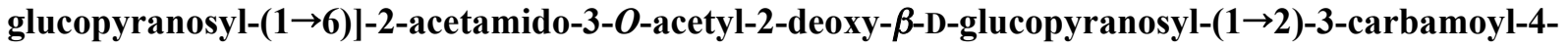
C-methyl- $\alpha$-D-glucopyranuronamide (16).

To a mixture of the above compound $(7.5 \mathrm{mg}, 5.01 \mu \mathrm{mol})$ in DMF $(1 \mathrm{ml})$ was added $\mathrm{H}_{2} \mathrm{NNH}_{2} \bullet \mathrm{HOAc}$ (0.94 mg, $10.0 \mu \mathrm{mol})$. After stirring for $1 \mathrm{~h}$, the solvent was evaporated and then the crude residue was purified by gradient reversed-phase chromatography, using a $0-100 \%$ gradient of $\mathrm{CH}_{3} \mathrm{CN} / \mathrm{H}_{2} \mathrm{O}$, to afford the title compound $(5.5 \mathrm{mg}, 3.78 \mu \mathrm{mol}, 75 \%)$ as a mixture $(\alpha: \beta, 3: 1) .{ }^{1} \mathrm{H}$ NMR $\left(600 \mathrm{MHz}, \mathrm{D}_{2} \mathrm{O}\right) \delta 5.74$ (br d, $\left.J=2.1 \mathrm{~Hz}, 1 \mathrm{H}, \mathrm{H}-4^{\mathrm{B}}\right), 5.52\left(\mathrm{~d}, J=3.5 \mathrm{~Hz}, 1 \mathrm{H}, \mathrm{H}-1^{\mathrm{F}}\right), 5.36\left(\mathrm{t}, J=9.4 \mathrm{~Hz}, 1 \mathrm{H}, \mathrm{H}-3^{\mathrm{D}}\right), 5.31(\mathrm{dd}, J=$ 3.6, $\left.10.0 \mathrm{~Hz}, 1 \mathrm{H}, \mathrm{H}-3^{\mathrm{B}}\right), 5.15-4.97\left(\mathrm{~m}, 6 \mathrm{H}, \mathrm{H}-3^{\mathrm{E}}, 4^{\mathrm{D}}, 3^{\mathrm{C}}, 2^{\mathrm{B}}, 1^{\mathrm{B}}, 3^{\mathrm{F}}\right), 4.87\left(\mathrm{~d}, J=7.9 \mathrm{~Hz}, 1 \mathrm{H}, \mathrm{H}-1^{\mathrm{D}}\right), 4.73-$ $4.69\left(\mathrm{~m}, 3 \mathrm{H}, \mathrm{H}-1^{\mathrm{C}}, 1^{\mathrm{E}}, 5^{\mathrm{B}}\right), 4.61-4.44\left(\mathrm{~m}, 2 \mathrm{H}, \mathrm{H}-5^{\mathrm{F}}, 6^{\mathrm{D}}\right), 4.27\left(\mathrm{~m}, 1 \mathrm{H}, \mathrm{H}-6^{\mathrm{D}}\right), 4.07-4.01(\mathrm{~m}, 2 \mathrm{H}, \mathrm{H}-$ $\left.5^{\mathrm{D}}, 6^{\mathrm{E}}\right), 3.90-3.62\left(\mathrm{~m}, 8 \mathrm{H}, \mathrm{H}-2^{\mathrm{C}}, 4^{\mathrm{C}}, 2^{\mathrm{E}}, 6^{\mathrm{E}}, 4^{\mathrm{E}}, 2^{\mathrm{F}}, 5^{\mathrm{E}}, 5^{\mathrm{C}}\right), 2.64\left(\mathrm{~s}, 4 \mathrm{H}, \mathrm{H}^{\mathrm{A}}\right), 2.16-1.97(\mathrm{~m}, 33 \mathrm{H}, 11 \mathrm{x} \mathrm{Ac})$, $1.30\left(\mathrm{~d}, \mathrm{~J}=6.2 \mathrm{~Hz}, 3 \mathrm{H}, \mathrm{H}-6^{\mathrm{C}}\right), 1.24(\mathrm{~s}, 3 \mathrm{H}, \mathrm{Me})$; LRMS calcd for $\mathrm{C}_{59} \mathrm{H}_{82} \mathrm{~N}_{5} \mathrm{O}_{37}[\mathrm{M}+\mathrm{H}]^{+}:$: 1452.4, found 1452.4 . 


\section{REFERENCES}

1. $\quad$ Lay, L.; Manzoni, L.; Schmidt, R. R. Carbohyd. Res. 1998, 310, 157-171.

2. Srivastava, G.; Alton, G.; Hindsgaul, O. Carbohyd. Res. 1990, 207, 259-276.

3. Jansson, K.; Ahlfors, S.; Frejd, T.; Kihlberg, J.; Magnusson, G.; Dahmén, J.; Noori, G.; Stenvall, K. J. Org. Chem. 1988, 53, 5629-5647.

4. $\quad$ Kartha, K. P. R.; Aloui, M.; Field, R. A. Tetrahedron Lett. 1996, 37, 8807-8810.

5. Hernández-Torres, J. M.; Achkar, J.; Wei, A. J. Org. Chem. 2004, 69, 7206-7211.

6. Debenham, J. S.; Madsen, R.; Roberts, C.; Fraser-Reid, B. J. Am. Chem. Soc. 1995, 117, 33023303.

7. Adachi, M.; Zhang, Y.; Leimkuhler, C.; Sun, B.; LaTour, J.; Kahne, D. J. Am. Chem. Soc. 2006, 128, 14012-14013. 